\title{
Modelos de Mistura para Dados de Sobrevivência na Presença de Covariáveis, Utilizando Métodos Bayesianos
}

\author{
Gilberto de Araújo Pereira \\ Orientador: Prof.Dr. Jorge Alberto Achcar
}

Dissertação apresentada ao Instituto de Ciências Matemáticas e de Computação, da USP, como parte dos requisitos para a obtenção do título de Mestre em Ciências-Área: Ciências de Computação e Matemática Computacional.

USP-São Carlos

Julho/1998 
Em especial aos amores de minha vida, Valdirene a (Val), meus pais e meus irmãos 


\section{Agradecimentos}

Acima de tudo, agradeço a Deus por me conceder a "vida" até o presente momento.

Ao Prof. Jorge Alberto Achcar, por conduzir este trabalho com admirável dedicação e disciplina, e especialmente por compartilhar comigo a sua "incontestável" experiência.

Aos Professores Jorge Oishi e Marinho Andrade pelas valiosas sugestões no exame de qualificação.

Aos colegas e funcionários do ICMSC-USP.

À FAPESP pelo apoio financeiro.

À Faculdade de Medicina do Triângulo Mineiro-FMTM, em especial aos integrantes do Centro de Graduação de Enfermagem pelo incentivo a este trabalho.

$\grave{A}$ minha amada Valdirene por fazer parte de todos os momentos importantes de minha vida, compartilhando comigo cada etapa vencida.

Aos meus pais, José Carlos e Rosa e meus irmãos Gislene e Gilson, por acreditarem em mim e sempre estarem ao meu lado.

Às minhas colegas Claudia e Silvana, pelo apoio e amizade no decorrer deste trabalho, e também ao meu colega Josmar pela contribuição no início deste trabalho.

Em especial ao meu amigo Miguel Eduardo, pela amizade e pelos 
preciosos ensinamentos sobre programação computacional para a realização deste trabalho.

Enfim, gostaria de agradecer a todos que direta ou indiretamente contribuiram para que este trabalho fosse realizado. 


\title{
Tẹminologia e Notação
}

Variáveis aleatórias são representadas por letras maiúsculas e suas realizações por minúsculas.

Os parâmetros do modelo são indicados por letras gregas.

As matrizes também são representadas por letras maiúsculas, os vetores por letras minúsculas negritadas, e as constantes também por minúsculas.

\begin{abstract}
A função densidade de probabilidade, função distribuição acumulada e probabilidade de incidência são representadas por $f_{j}\left(\bullet \mid \mathbf{x}, \beta_{\mathbf{j}}\right), F_{j}\left(\bullet \mid \mathbf{x}, \beta_{\mathbf{j}}\right)$ e $P_{j}\left(\bullet \mid \mathbf{x}, \gamma_{\mathbf{j}}\right)$, respectivamente, a densidade à priori por $\pi(\bullet)$ e a densidade à posteriori por $\pi(\bullet \mid \mathbf{x}, \Theta)$.
\end{abstract}

Utilizamos as seguintes abreviações:

F.V.: Função de Verossimilhança

E.M.V.: Estimador de Máxima Verossimilhança

MCMC: Cadeia de Markov com Monte Carlo

iid: Independente e identicamente distribuídos 


\section{Abstract}

In this dissertation, we present a Bayesian analysis of distributions finite mixture models, for survival data uncensored, type II censoring and interval-censored data, in the presence of one covariate. Considering Gibbs sampling with Metropolis-Hastings algorithms, we get Monte Carlo estimates for the posterior quantities of interest, assuming different choices for the $(J=2)$ densities in the mixture model, for example a mixture, two exponential power distributions which includes a wider class of symmetric distributions, two normal distributions, normal-exponential and gammanormal distributions. We also present some considerations on model selection, considering the predictive densities (CPO) conditional predictive ordinate, and we introduce three numerical example to illustrate the proposed methodology. 


\section{Resumo}

Nesta dissertação, desenvolvemos uma análise Bayesiana de modelos de mistura finita de distribuições, para dados de sobrevivência sem censura, com censura tipo II e dados censurados por intervalos, na presença de uma covariável. Consideramos os algoritmos amostrador de Gibbs com Metropolis-Hastings, e utilizamos os estimadores de Monte Carlo para conseguir as quantitades à posteriori de interesse, assumindo diferentes escolhas para as $(J=2)$ densidades no modelo de mistura, como por exemplo a mistura de, duas distribuições potência exponencial a qual considera uma grande classe de distribuições simétricas, duas distribuições normais, normal-exponencial e gamma-normal. Apresentamos também algumas considerações na seleção do modelo utilizando as densidades preditivas (CPO)preditivas condicionais ordenadas e introduzimos três exemplos numéricos para ilustrar a metodologia proposta. 


\section{Conteúdo}

1 Introdução 1

1.1 Modelo de Mistura em Análise de Sobrevivência ............2

1.2 Motivações e Objetivos ............................. 5

1.3 Organização do Trabalho $\ldots \ldots \ldots \ldots \ldots \ldots \ldots \ldots \ldots \ldots \ldots \ldots \ldots$

2 Função Verossimilhança e Variáveis Artificiais 13

2.1 Função de Verossimilhança $\ldots \ldots \ldots \ldots \ldots \ldots \ldots \ldots \ldots \ldots \ldots$

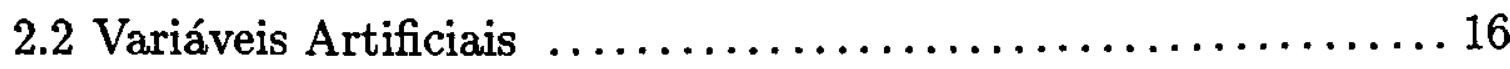

3 Modelos de Mistura para Dados sem Censuras 23

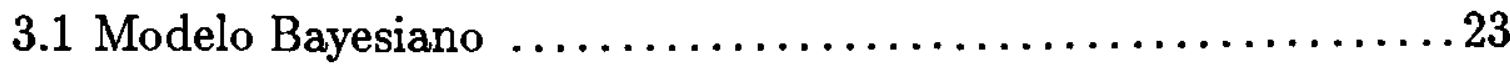

3.2 Algoritmos de Simulação de Amostras $\ldots \ldots \ldots \ldots \ldots \ldots \ldots 26$

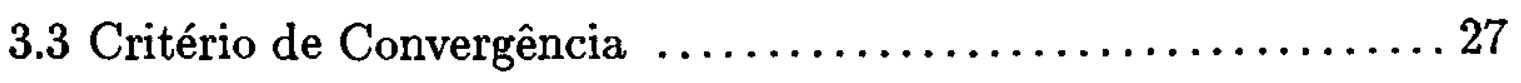

3.4 Mistura das Distribuições Potência Exponencial ...........28

3.5 Mistura das Distribuições Normal-Exponencial ............35

3.6 Mistura de Duas Distribuições Normais ................ 38

3.7 Mistura das Distribuições Gamma-Normal .............. 42

4 Modelos de Mistura para Dados com Censura Tipo II $\quad 47$ 
4.1 Mistura das Distribuições Potência Exponencial ............48

4.2 Mistura das Distribuições Normal-Exponencial ............55

4.3 Mistura de Duas Distribuições Normais ................ 58

4.4 Mistura das Distribuições Gamma-Normal ..............61

5 Modelos de Mistura para Dados Agrupados 65

5.1 Mistura das Distribuições Potência Exponencial ...........66

5.2 Mistura das Distribuições Normal-Exponencial ...........68

5.3 Mistura de Duas Distribuições Normais ................69 69

5.4 Mistura das Distribuições Gamma-Normal ..............69

5.5 Algumas Considerações na Seleção do Modelo .............70

6 Exemplos de Aplicações

72

6.1 Exemplo 1: Dados Observados Completamente ......73

6.1.1 Mistura de Duas Distribuições Potência Exponencial ......75

6.1.2 Mistura de Duas Distribuições Normais .............. 80

6.1.3 Mistura das Distribuições Normal-Exponencial ..........82

6.1.4 Mistura das Distribuições Gamma-Normal $\ldots \ldots \ldots \ldots \ldots . \ldots 3$

6.1.5 Discriminação dos Modelos $\ldots \ldots \ldots \ldots \ldots \ldots \ldots \ldots \ldots \ldots 6 . \ldots \ldots$ 


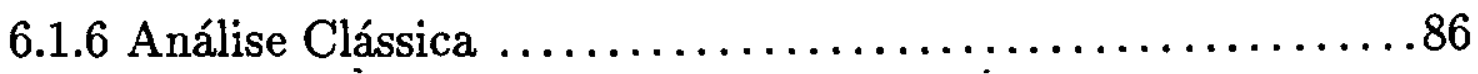

6.2 Exemplo 2: Dados com Censura Tipo II $\ldots \ldots \ldots \ldots 87$

6.2.1 Mistura de Duas Distribuições Potência Exponencial .....89

6.2.2 Mistura de Duas Distribuições Normais . .............91

6.2.3 Mistura das Distribuições Normal-Exponencial ..........92

6.2.4 Mistura das Distribuições Gamma-Normal .............94

6.2.5 Discriminação dos Modelos ......................95

6.3 Exemplo 3: Dados com Censura por Intervalos ......96

6.3.1 Mistura de Duas Distribuições Potência Exponencial ......98

6.3.2 Mistura das Distribuições Normal-Exponencial ..........100

7 Conclusōes e Considerações Futuras 103

Apêndice A: Função Distribuição Acumulada da Potência Exponencial ..............................105

Apêndice B: Agoritmo Metropolis-Hastings ............ 108

Apêndice C: Critério de Convergência $\ldots \ldots \ldots \ldots \ldots \ldots \ldots \ldots \ldots \ldots \ldots \ldots \ldots$

Apêndice D: Programa Computacional ................113

Bibliografia $\ldots \ldots \ldots \ldots \ldots \ldots \ldots \ldots \ldots \ldots \ldots \ldots \ldots \ldots \ldots \ldots \ldots$ 


\section{Capítulo 1}

\section{Introdução}

A constante necessidade de uma modelagem mais ampla para interpretar fenômenos das mais diversas áreas do conhecimento humano, como agricultura, biologia, economia, genética, medicina, etc..., tem atraído grande interesse para o desenvolvimento metodológico de modelos de mistura de distribuições pelos estatísticos, (ver por exemplo, Everitt e Hand (1981), Titterington et.al. (1985) e McLachlan e Basford (1988)). Embora existam fenômenos que permitam uma modelagem probabilística diretamente através das distribuições clássicas como, normal, gamma, poisson, binomial, etc..., muitos outros fenômenos devido a sua grande complexidade, só podem ser estudados à partir de hiper-modelos.

A razão fundamental para o interesse em mistura de distribuições é que esses modelos proporcionam alternativas interessantes à modelagem não paramétrica, e menos restrições do que as distribuições usuais. Localizado entre estes dois extremos, estes modelos desfrutam simultaneamente, de uma grande liberdade e da simplicidade da modelagem paramétrica. 


\subsection{Modelo de Mistura em Análise de Sobrevivência}

Direcionando a atenção para a área biomédica, mais especificamente em análise de sobrevivência, experimentos são realizados e na maioria das vezes, resultam em dados de modelagens complexas e de difícil análise do ponto de vista da estatística clássica. Por exemplo, ao analisar o tempo de vida de uma determinada população de indivíduos expostos a um certo tipo de tratamento, podemos encontrar algumas subpopulações sensíveis e outras insensíveis ao tratamento, ou seja, o tempo de vida da população assume uma forma multimodal (veja figura 1.1).

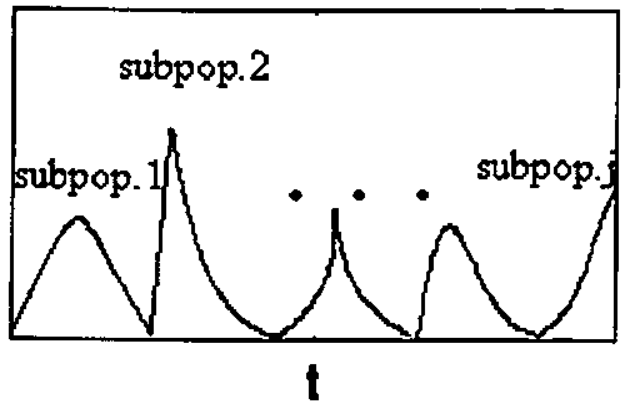

Figura 1.1: tempo de vida de indivíduos expostos à um determinado tratamento

Portanto, em vez de assumirmos apenas uma função densidade de probabilidade para explicar os dados, consideramos um modelo de mistura que incorpore diferentes distribuições de latências, várias covariáveis que podem influenciar nas probabilidades de incidência e nas distribuições de latências condicionais, para as diferentes subpopulações.

Usualmente podemos ter grande dificuldade em obter inferências para os parâmetros do modelo de mistura usando métodos frequentistas usuais. 
Uma alternativa que tem sido explorada por muitos estatísticos é o uso de métodos Bayesianos, especialmente algoritmos computacionais de simulação do tipo MCMC (Cadeia de Markov com Monte Carlo), para a obtenção das quantidades à posteriori de interesse (ver por exemplo, Chib e Greenberg, 1994).

Uma das críticas ao uso de métodos Bayesianos em modelos de misturas é que podemos ter grande tempo de trabalho computacional para problemas com muitos parâmetros. No entanto, uma aproximação baseada numa amostragem por simulação estocástica tem fácil implementação e é razoavelmente eficiente ( ver por exemplo, Geweke, 1989; Gelfand e Smith, 1990 ou Tierney, 1994).

Consideramos um modelo da família paramétrica, para o tempo de sobrevivência $t$, de mistura finita de distribuições, isto é, uma família de probabilidades com função densidade da forma:

$$
f(\mathbf{t} \mid \mathbf{x}, \Theta)=\sum_{j=1}^{J} P\left(y=j \mid \mathbf{x}, \gamma_{\mathbf{j}}\right) f_{j}\left(\mathbf{t} \mid y=j, \mathbf{x}, \beta_{\mathbf{j}}\right)
$$

O lado direito de (1.1) é chamado de mistura finita de distribuições.

onde:

$\mathbf{t}:$ vetor dos tempos de sobrevivência associados a cada indivíduo $i$;

$\mathbf{x}$ : vetor de covariáveis associadas à um indivíduo com tempo de vida $\mathbf{t}$

$\Theta=\left[\beta_{1}, \beta_{2}, \ldots, \beta_{j}, \gamma_{1}, \gamma_{2}, \ldots, \gamma_{j}\right]$ : é o conjunto de todos os vetores 
de parâmetros envolvidos no modelo (1.1);

$y$ : variável índice para a subpopulação;

$f_{j}$ : é a função densidade de probabilidade condicional ao tempo de vida para a $j$-ésima subpopulação homogênea, e está indexada por um vetor de parâmetros contínuos desconhecidos $\beta_{\mathrm{j}}$.

As probabilidades de mistura $P\left(y=j \mid \mathbf{x}, \gamma_{\mathbf{j}}\right)$, associadas às covariáveis e indexada por um vetor de parâmetros desconhecidos $\gamma_{\mathbf{j}}$, abreviada por $P\left(j \mid \mathbf{x}, \gamma_{\mathbf{j}}\right)$, também conhecida por probabilidades de incidência, devem satisfazer $\sum_{j=1}^{J} P\left(j \mid \mathbf{x}, \gamma_{\mathbf{j}}\right)=1$.

Os modelos de regressão logística, usualmente são escolhidos para modelar as probabilidades de mistura. Seja $\xi_{j}$ o produto interno de $\mathbf{x}$ e $\gamma_{\mathbf{j}}$, tal que, $\xi_{j}=\mathbf{x} \gamma_{\mathbf{j}}^{\mathbf{T}}$ onde $\gamma_{\mathbf{j}}^{\mathbf{T}}$ denota o vetor transposto de $\gamma_{\mathbf{j}}(1 \mathrm{x} \mathrm{p}$ ), (ver por exemplo Cox, 1970), então um modelo para explicar as probabilidades de incidência é dado por,

$$
P\left(j \mid \mathbf{x}, \gamma_{\mathbf{j}}\right)=\frac{e^{\mathbf{x} \gamma_{j}^{\mathbf{T}}}}{\sum_{j=1}^{J} e^{\mathbf{x} \gamma_{j}^{\mathbf{T}}}}
$$

Outros modelos tais como o probito normalizado ou o complementar normalizado (ver por exemplo, McCullagh e Nelder, 1989), também podem ser considerados para parametrizar as probabilidades de incidência.

A função distribuição acumulada de t, derivada de (1.1) é dada por: 


$$
F(\mathbf{t} \mid \mathbf{x}, \Theta)=\sum_{j=1}^{J} P\left(j \mid \mathbf{x}, \gamma_{\mathbf{j}}\right) F_{j}\left(\mathbf{t} \mid \mathbf{x}, \beta_{\mathbf{j}}\right)
$$

onde, $F_{j}$ é a função distribuição de $f_{j}$.

\subsection{Motivações e Objetivos}

Em modelos de mistura, frequentemente o estimador usual de máxima verossimilhança (E.M.V.) é difícil de ser encontrado seja qual for o tamanho amostral, mesmo para casos simples como mistura de duas distribuições normais (ver por exemplo, Lehmann 1983 ou Robert, 1996). Isto acontece porque, para uma dada amostra $t_{1}, t_{2}, \ldots, t_{n}$ modelada por (1.1), há sempre uma probabilidade positiva de que um componente $f_{j}(\mathbf{t} \mid \mathbf{x}, \Theta)$ não explica de modo algum as observações $\left(t_{j}\right)$, ou seja, a amostra não traz informação sobre qual dos componentes de mistura em particular a observação $\left(t_{j}\right)$ pertence. Este fenômeno não necessariamente impede a obtenção do E.M.V., mas contribui muito para sua instabilidade. Este problema peculiar de identificabilidade do modelo de mistura, motiva o uso de métodos Bayesianos para produzir estimadores aceitáveis e idealizar testes sobre o número de componentes de mistura (ver por exemplo, Dey, Kuo e Sahu, 1995).

Modelos de mistura foram considerados por Farewell (1982), Larson e Dinse (1986), Pack e Morgan (1990), Boos e Brownie (1991), Kuk e Chen (1992) e Taylor (1994). No artigo de Boos e Brownie (1991), os tempos de sobrevivência foram considerados independentes e identicamente distribuídos (iid), e modelado por (1.1). Pack e Morgan (1990) consideram 
os tempos censurados por intervalo $\left(t_{i l}, t_{i u}\right)$ e à direita em $\left(t_{i h}\right)$. Larson e Dinse (1986), Kuk e Chen (1992) combinam uma formulação logística para as probabilidades de incidência com uma especificação proporcional padrão (por exemplo, Normal, Gamma, Exponencial, Weibull) para as distribuições de latências condicionais e Taylor (1994) considera o tipo de formulação de Kaplan-Meier.

$\mathrm{Na}$ literatura, muitos outros autores têm considerado modelos de mistura para analisar tempos de sobrevivência. Com misturas das distribuições, Normal (ver por exemplo, Quandt e Ramsey, 1978), Exponencial (ver por exemplo, Mendenhall e Hader, 1958; Tallis e Light, 1968)e Gamma (ver por exemplo, Dickinson, 1974).

Box e Tiao (1973) consideram a distribuição potência exponencial como densidade para os dados em certas análises Bayesianas, como por exemplo, testar o efeito de não normalidade dos dados em modelos simples. Com o mesmo propósito consideramos essa distribuição no modelo de mistura (1.1), a qual pode trazer grande flexibilidade para analisar tempos de sobrevivência, especialmente se distribuições aproximadamente simétricas podem ser consideradas. A distribuição potência exponencial tem densidade dada por:

$$
f_{j}\left(\mathbf{t} \mid \mathbf{x}, \beta_{\mathbf{j}}\right)=\frac{w\left(\delta_{j}\right)}{\sigma_{j}} \exp \left\{-c\left(\delta_{j}\right)\left|\frac{t_{i}-\theta_{j}}{\sigma_{j}}\right|^{2 /\left(1+\delta_{j}\right)}\right\}
$$

onde $-\infty<t_{i}<\infty ; \mathrm{j}=1,2 ; \beta_{\mathrm{j}}=\left(\delta_{j}, \sigma j, \theta_{j}\right) ; \mathrm{e}$, 


$$
\begin{aligned}
& c\left(\delta_{j}\right)=\left\{\frac{\Gamma\left[\frac{3}{2}\left(1+\delta_{j}\right)\right]}{\Gamma\left[\frac{1}{2}\left(1+\delta_{j}\right)\right]}\right\}^{1 /\left(1+\delta_{j}\right)} \\
& w\left(\delta_{j}\right)=\frac{\left\{\Gamma\left[\frac{3}{2}\left(1+\delta_{j}\right)\right]\right\}^{1 / 2}}{\left(1+\delta_{j}\right)\left\{\Gamma\left[\frac{1}{2}\left(1+\delta_{j}\right)\right]\right\}^{3 / 2}}
\end{aligned}
$$

$\sigma_{j}>0 ;-1<\delta_{j} \leq 1$ e $-\infty<\theta_{j}<\infty$

A função distribuição acumulada de (1.4) pode ser expressa como a integral de uma gamma imcompleta,

$$
\begin{aligned}
F_{j}\left(\mathbf{t} \mid \mathbf{x}, \beta_{\mathbf{j}}\right)= & \left\{\frac{\left(1+\delta_{j}\right) w\left(\delta_{j}\right) \Gamma\left(\frac{\delta_{j}+1}{2}\right)}{\left[c\left(\delta_{j}\right)\right]^{\left(\delta_{j}+1\right) / 2}}\right\} \\
& I_{\frac{\left(\delta_{j}+1\right)}{2}}\left\{c\left(\delta_{j}\right)\left|\frac{t_{i}-\theta_{j}}{\sigma_{j}}\right|^{2 /\left(1+\delta_{j}\right)}\right\}
\end{aligned}
$$

onde

$$
I_{k}(s)=\frac{1}{\Gamma(k)} \int_{0}^{s} x^{k-1} e^{-x} d x
$$

ver Demonstração (Apêndice A)

A densidade (1.4) considera em sua forma uma grande classe de distribuições simétricas onde a distribuição normal está inclusa quando $\delta_{j}=0$; ao mesmo tempo outras distribuições mais leptocúrticas quando $\delta_{j}>0$, ou mais platicúrticas quando $\delta_{j}<0$ são consideradas, ou seja,

(i) Quando $\delta_{j}=0$ a densidade (1.4) assume a forma de uma dis- 
tribuição normal,

$$
f_{j}\left(\mathbf{t} \mid \mathbf{x}, \beta_{\mathbf{j}}\right)=\frac{1}{\sqrt{2 \pi} \sigma_{j}} \exp \left\{-\frac{1}{2 \sigma_{j}^{2}}\left|t_{i}-\theta_{j}\right|^{2}\right\},
$$

$-\infty<t_{i}<+\infty$, veja figura (1.2),

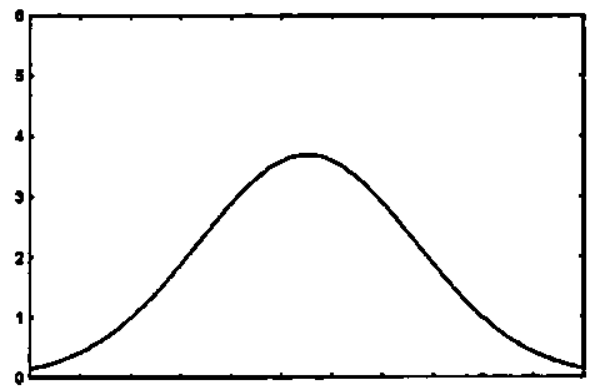

Figura 1.2: Distribuição Potência Exponencial para $\delta_{j}=0$ (Normal)

(ii) Quando $\delta_{j}=1$ a densidade (1.4) assume uma distribuição exponêncial dupla,

$$
f_{j}\left(\mathbf{t} \mid \mathbf{x}, \beta_{\mathbf{j}}\right)=\frac{1}{\sqrt{2} \sigma_{j}} \exp \left\{-\sqrt{2}\left|t_{i}-\theta_{j}\right|\right\}
$$

$-\infty<t_{i}<+\infty$, veja figura (1.3) 


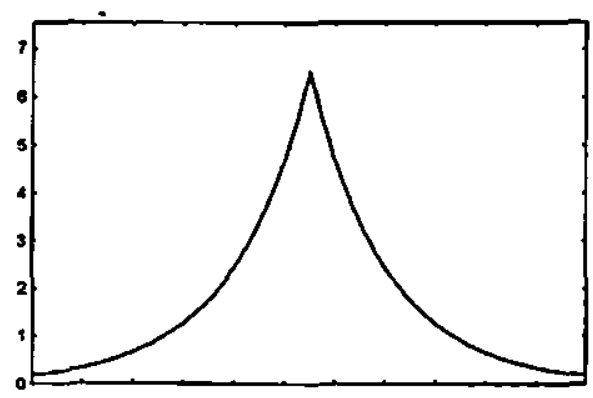

Figura 1.3: Distribuição Potência Exponencial para $\delta_{j}=1$ (Exponencial Dupla)

(iii) Quando $\delta_{j} \rightarrow-1$ a densidade (1.4) tende para uma distribuição retangular,

$$
\lim _{\delta_{j} \rightarrow-1} f_{j}\left(\mathbf{t} \mid \mathbf{x}, \beta_{\mathbf{j}}\right)=\frac{1}{2 \sqrt{3} \sigma_{j}}
$$

$\theta_{j}-\sqrt{3} \sigma_{j}<t_{i}<\theta_{j}+\sqrt{3} \sigma_{j}$, veja figura (1.4),

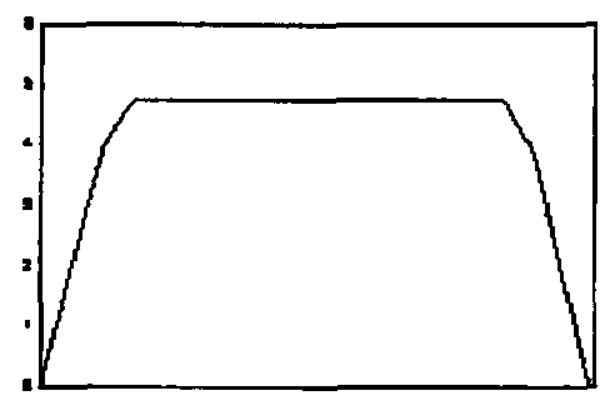

Figura 1.4: Distribuição Potência Exponencial para $\delta_{j} \rightarrow-1$ (Retangular)

Nesta dissertação, temos como objetivo principal obter estimativas de todos os parâmetros envolvidos no modelo de mistura realizando uma análise Bayesiana e, para isso, tempos de sobrevivência sem censura, com censura tipo II e censura por intervalos são considerados. O desenvolvi- 
mento desta análise envolve o cálculo do produto de mistura de densidades, e em se tratando de observações censuradas, precisamos calcular integrais da mistura de densidades. Assim, para resolver estes problemas uma alternativa viável é a utilização de métodos computacionais Bayesianos. Usamos neste trabalho os algoritmos de simulação estocástica como o amostrador de Gibbs e Metropolis-Hastings baseados na teoria de Cadeia de Markov (ver por exemplo, Diebolt e Robert, 1994). A implementação computacional desses algoritmos é simples se comparado a outros métodos Bayesianos propostos, como por exemplo o método de Laplace (ver, Tierney e Kadane, 1986), e o tempo computacional é razoável, considerando a complexidade do modelo.

Uma das primeiras aplicações do algoritmo MCMC em modelos de mistura para observações iid foi considerado por Diebolt e Robert (1994). Kuo e Peng (1995) extenderam o uso desses algoritmos para o caso de dados censurados. $\mathrm{O}$ princípio básico dessa extensão pode ser visto em Gelfand, Smith e Lee (1992) e Kuo e Smith (1992).

Um dos problemas em modelos de mistura é determinar o número de componentes de mistura $J$ (subpopulação). Na maioria das análises de modelos de mistura, $J$ é assumido como conhecido. Mas se $J$ não é conhecido e não aleatório, podemos determina-lo através de uma densidade preditiva aproximada (ver por exemplo, Dey, Kuo e Sahu, 1995), onde cada valor distinto de $J$ origina-se num novo modelo estatístico. A distribuição preditiva sugere, para um certo conjunto de dados, como obter um valor apropriado de $J$.

Neste trabalho consideramos $J=2$ conhecido, e no modelo (1.1) algumas misturas de distribuições, (Normal-Exponencial), (Gamma-Normal), 
mistura de duas Normais e mistura de duas distribuições Potência Exponencial, para analisar tempos de vida $t$.

Devido a esse razoável número de distribuições de latência condicionais que introduzimos no modelo de mistura (1.1) para explicar os tempos de vida dos indivíduos, necessitamos selecionar qual mistura de distribuições melhor se ajusta aos dados para os três diferentes esquemas amostrais (sem censura, censura tipo II e por intervalo). $\mathrm{O}$ uso da densidade preditiva tem sido considerado como uma boa aproximação Bayesiana para selecionar o melhor modelo (ver por exemplo, Kuo e Peng, 1995). Em particular, Box (1980) notifica o papel complementar da distribuição à posteriori e distribuição preditiva, argumentando que a posteriori é usada para "estimação de parâmetros condicionais na adequação do modelo", enquanto que a preditiva é usada para verificar se um modelo é adequado para os dados.

Com o objetivo de confrontar as estimativas dos parâmetros baseado na teoria Bayesiana com as obtidas pela teoria Clássica, uma análise clássica também foi considerada para os modelos de mistura, em alguns exemplos.

\subsection{Organização do Trabalho}

Esta dissertação está organizada em 7 capítulos. Neste capítulo introdutório apresentamos o modelo de mistura finita de distribuições, as motivações e os objetivos do trabalho.

No segundo capítulo, apresentaremos as funções de Verossimilhança 
para o caso de dados sem censura, censura tipo II e censura por intervalos, e também as variáveis artificiais que são introduzidas no modelo de mistura afim de facilitar a implementação dos algoritmos de simulação de amostras, amostrador de Gibbs e Metropolis-Hastings.

No capítulo 3, serão apresentados, o algoritmo amostrador de Gibbs, Critério de Convergência de Gelman e Rubin, e também nos capítulos 3, 4 e 5 apresentaremos os modelos de mistura para dados sem censuras, com censura tipo II e censura por intervalos, respectivamente, considerando as seguintes misturas:

- Mistura das Distribuições Potência Exponencial

- Mistura das Distribuições Normal e Exponencial

- Mistura das Distribuições Normais

- Mistura das Distribuições Gamma e Normal

Os exemplos numéricos para ilustrar a metodologia proposta, serão apresentados no capítulo 6 , onde desenvolvemos uma análise Bayesiana considerando para o caso de dados sem censura e com censura tipo II dados gerados à partir de Hewlett (1974), e para o caso de dados agrupados consideramos um conjunto de dados reais introduzido por Hewlett (1974). As conclusões e considerações futuras apresentaremos no capítulo 7 .

Nos Apêndices A, B, C e D, serão apresentados, a demostração da função distribuição acumulada da Potência Exponencial, Agoritmo Metropolis-Hastings, Critério de Convergência de Gelman e Rubin e Programa computacional, respectivamente. 


\section{Capítulo 2}

\section{Função de Verossimilhança e Variáveis Artificiais}

\subsection{Função de Verossimilhança}

Toda informação contida nos dados à respeito dos parâmetros do modelo é expressa pela função de verossimilhança (F.V.) dos dados, e podemos escreve-la para os diferentes esquemas amostrais.

i) Se o tempo de vida de cada indivíduo for observado exatamente (sem censura), veja esquema na figura (2.1),

a F.V. para o modelo de mistura pode ser escrita da seguinte forma:

$$
L(\Theta \mid \mathbf{t}, \mathbf{x})=\prod_{i=1}^{n}\left\{\sum_{j=1}^{J} P\left(j \mid \mathbf{x}, \gamma_{\mathbf{j}}\right) f_{j}\left(t_{i} \mid \mathbf{x}, \beta_{\mathbf{j}}\right)\right\}
$$

ii) Se o tempo de vida o $i$-ésimo indivíduo for censurado à direita de 


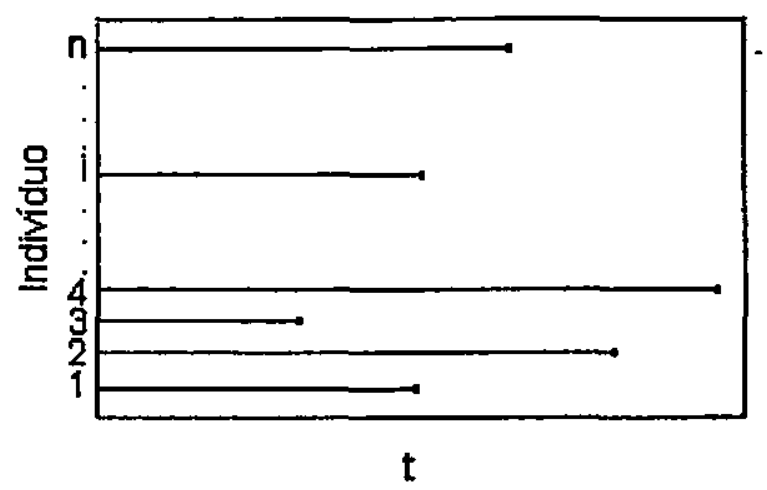

Figura 2.1: Tempos de vida dos indivíduos observados completamente (sem censura)

$t_{i h}$, isto é, dos $n$ indivíduos expostos ao tratamento observamos os tempos de vida até que $r$ indivíduos morrem, e os outros $(n-r)$ são censurados (censura tipo II), veja esquema na figura (2.2),

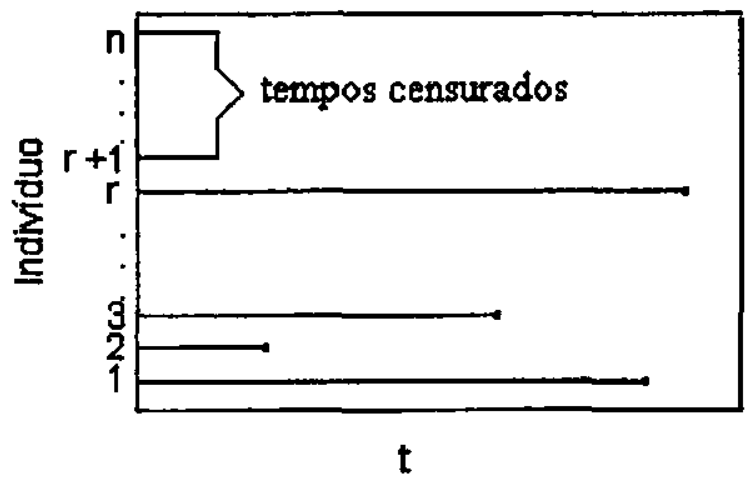

Figura 2.2: Tempos de vida dos indivíduos com censura tipo II

temos então a seguinte contribuição da parte censurada para a F.V.:

$$
\mathcal{S}^{(n-r)}\left(t_{i h} \mid \mathbf{x}, \Theta\right)=\{P(T>t)\}^{(n-r)}=\left\{1-F\left(t_{i h} \mid \mathbf{x}, \Theta\right)\right\}^{(n-r)}
$$

onde,

$(n-r)$ : são os indivíduos censurados à direita em $t_{i h}, \mathrm{e}$

$F\left(t_{i h} \mid \mathrm{x}, \Theta\right)$ definido em (1.3) 
Portanto a F.V. pode ser escrita da seguinte forma:

$$
\begin{aligned}
L(\Theta \mid \mathbf{t}, \mathbf{x})= & \prod_{i=1}^{n_{i}}\left\{\sum_{j=1}^{J} P\left(j \mid \mathbf{x}, \gamma_{\mathbf{j}}\right) f_{j}\left(t_{i} \mid \mathbf{x}, \beta_{\mathbf{j}}\right)\right\} \\
& \prod_{i=n_{i}+1}^{n}\left\{1-\left[\sum_{j=1}^{J} P\left(j \mid \mathbf{x}, \gamma_{\mathbf{j}}\right) F_{j}\left(t_{i h} \mid \mathbf{x}, \beta_{\mathbf{j}}\right)\right]\right\}
\end{aligned}
$$

iii) Se os tempos de vida dos $n_{i}$ primeiros indivíduos forem censurados por intervalo, ou seja, com o $i$-ésimo indivíduo morrendo entre os tempos $\left(t_{i l}, t_{i u}\right)$, e os $\left(n-n_{i}\right)$ indivíduos restantes censurados à direita de $t_{i h}$, veja esquema na figura (2.3),

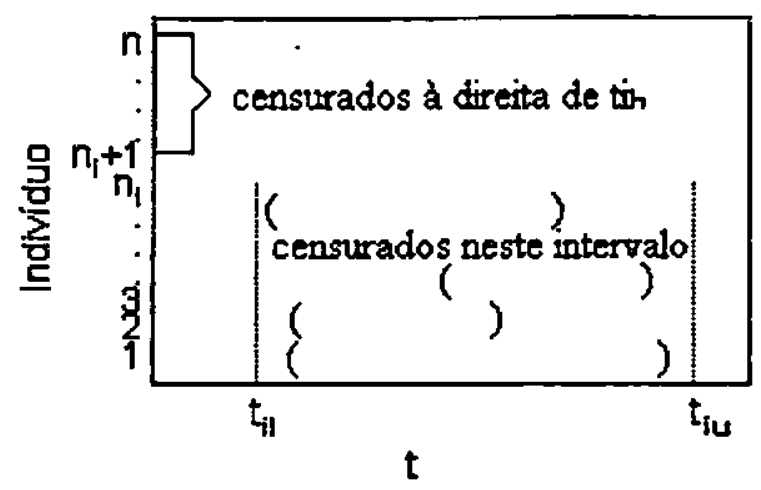

Figura 2.3: Tempos de vida dos indivíduos censurados por intervalo e à direita teremos a seguinte contribuição das partes censuradas para a F.V.:

$$
\underbrace{\left\{F\left(t_{i u} \mid \mathbf{x}, \Theta\right)-F\left(t_{i l} \mid \mathbf{x}, \Theta\right)\right\}}_{\text {censurado no intervalo }\left(t_{i l}, t_{i u}\right)} \underbrace{\left\{1-F\left(t_{i h} \mid \mathbf{x}, \Theta\right)\right\}}_{\text {censurado à direita de } t_{i h}}
$$

onde,

$F\left(t_{i l} \mid \mathbf{x}, \Theta\right) ; F\left(t_{i u} \mid \mathbf{x}, \Theta\right) ;$ e $F\left(t_{i h} \mid \mathbf{x}, \Theta\right)$ são definidos como em (1.3) Assim, a F.V. para este esquema amostral pode ser escrita por, 


$$
\begin{aligned}
L(\Theta \mid \mathbf{t}, \dot{\mathbf{x}})= & \prod_{i=1}^{n_{i}}\left\{F\left(t_{i u} \mid \mathbf{x}, \Theta\right)-F\left(t_{i l} \mid \mathbf{x}, \Theta\right)\right\} \\
& \prod_{i=n_{i}+1}^{n}\left\{1-F\left(t_{i h} \mid \mathbf{x}, \Theta\right)\right\}
\end{aligned}
$$

\subsection{Variáveis Artificiais}

Como podemos observar, a parte correspondente aos dados censurados nas F.V. (2.2) e (2.3), são produtos de integrais da mistura de distribuições, e calcular as distribuições à posteriori marginais de $\Theta$ em se tratando de uma análise Bayesiana, é bastante complexo.

Para simplificar as distribuições à posteriori, consideramos dois grupos de variáveis artificiais (ver por exemplo, Tanner e Wong, 1987), um que leva uma F.V. de dados incompletos para uma F.V. de dados iid, (Variável Artificial $W$ ), e outro grupo que transforme o modelo de mistura em um modelo de componentes independentes (Variável Artificial $Z$ ). Assim, o cálculo das densidades marginais à posteriori de $\Theta$, necessárias para a implementação dos algoritmos de simulação de amostras do tipo MCMC, são bastante facilitados considerando as variáveis artificiais $W$ e $Z$, respectivamente.

Desta forma, geramos independentemente para cada observação, $i=$ $1, \ldots, n$, um componente $\left(w_{i}\right)$ da variável $W$, que é truncada em $t_{i h}$ se a observação for censurada à direita; truncada entre $\left(t_{i l}, t_{i u}\right)$ se censurado nesse intervalo e $w_{i}=t_{i}$ se for observado exatamente (sem censura) (ver por exemplo, Kuo e Peng, 1995). Portanto a densidade conjunta dos dados 
e as variáveis artificiais terão componentes iid, cada um distribuído como em (1.1), o que elimina o cálculo de integrais no modelo de mistura; em resumo, temos:

i) se o tempo de vida do i-ésimo indivíduo for observado exatamente em $t_{i}$, consideramos $w_{i}=t_{i}$ e a F.V. pode ser escrita como em (2.1);

ii) se o tempo de vida do i-ésimo indivíduo for censurado à direita em $t_{i h}$, então podemos gerar $w_{i}$ da densidade truncada:

$$
f\left(w_{i}\right) /\left[1-F\left(t_{i h}\right)\right], I\left[t_{i h}<w_{i}<\infty\right]
$$

ou seja,

$$
w_{i}=F^{-1}\left\{F\left(t_{i h} \mid \mathbf{x}, \Theta\right)+U\left[1-F\left(t_{i h} \mid \mathbf{x}, \Theta\right)\right]\right\}
$$

onde: $U \sim U(0,1) ; F\left(t_{i h} \mid \mathbf{x}, \Theta\right)$ é difịido como em (1.3); e $F^{-1}$ é a função inversa de $F$.

Como nem sempre conseguimos obter analiticamente a $F^{-1}$, dado $\mathbf{x}, t_{i h}$ e $\Theta$, fazemos,

- calculamos $F\left(t_{i h} \mid \mathbf{x}, \Theta\right)$ de $(1.3)$

- geramos um valor de $U$ de uma distribuição $U(0,1)$;

- calculamos $v_{i}=F\left(t_{i h} \mid \mathbf{x}, \Theta\right)+U\left[1-F\left(t_{i h} \mid \mathbf{x}, \Theta\right)\right]$, na forma, $w_{i}=F^{-1}\left(v_{i}\right)$, ou seja $w_{i}$ é a única raiz de $F\left(w_{i}\right)=v_{i}$, veja figura (2.4),

Para encontrar os valores de $w_{i}$ utilizamos o método numérico de New- 


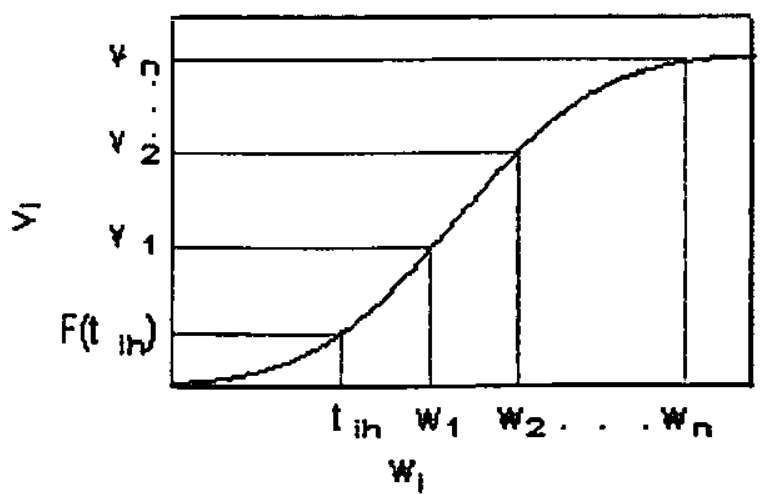

Figura 2.4: raizes de $w_{i}$ para o caso de censura à direita

ton. Geramos $w_{i}$ independentemente para cada observação, portanto a F.V. (2.2), dado a introdução da variável artificial $W$, pode ser escrita por:

$$
L(\Theta \mid W, \mathbf{x})=\prod_{i=1}^{n}\left\{\sum_{j=1}^{J} P\left(j \mid \mathbf{x}, \gamma_{\mathbf{j}}\right) f_{j}\left(w_{i} \mid \mathbf{x}, \beta_{\mathbf{j}}\right)\right\}
$$

iii) se o tempo de vida do $i$-ésimo indivíduo for censurado no intervalo $\left(t_{i l}, t_{i u}\right)$, então podemos gerar $w_{i}$ a partir da densidade truncada:

$$
f\left(w_{i}\right) /\left[F\left(t_{i u}\right)-F\left(t_{i l}\right)\right], I\left[t_{i l}<w_{i}<t_{i u}\right]
$$

ou seja,

$$
w_{i}=F^{-1}\left\{F\left(t_{i u} \mid \mathbf{x}, \Theta\right)+U\left[F\left(t_{i u} \mid \mathbf{x}, \Theta\right)-F\left(t_{i l} \mid \mathbf{x}, \Theta\right)\right]\right\}
$$

onde:

$U \sim U(0,1) ; F\left(t_{i l} \mid \mathbf{x}, \Theta\right)$ e $F\left(t_{i u} \mid \mathbf{x}, \Theta\right)$ são definidos como em (1.3); e $F^{-1}$ é a função inversa de $F$. 
Portanto dado $\mathbf{x}, t_{i l}, t_{i u}$ e $\Theta$, temos,

- calculamos $F\left(t_{i l} \mid \mathbf{x}, \Theta\right)$ e $F\left(t_{i u} \mid \mathbf{x}, \Theta\right)$; de (1.3)

- geramos um valor de $U$ de uma distribuição $U(0,1)$;

- calculamos $\tilde{v}_{i} \mid=F\left(t_{i l} \mid \mathbf{x}, \Theta\right)+U\left[F\left(t_{i u}-F\left(t_{i l} \mid \mathbf{x}, \Theta\right)\right]\right.$; assim, $w_{i}=F^{-1}\left(v_{i}\right)$, isto é, $w_{i}$ é a única raiz de $F\left(w_{i}\right)=v_{i}$, veja figura (2.5),

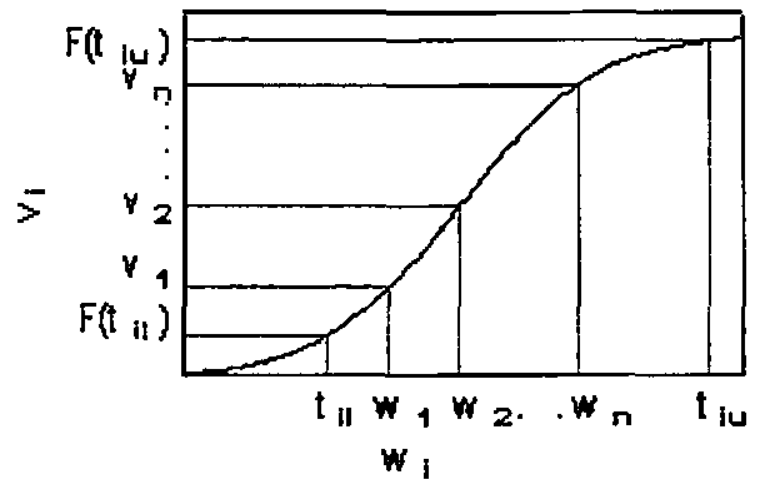

Figura 2.5: raizes de $w_{i}$ para o caso de censura por intervalo

Para encontrar os valores de $w_{i}$ utilizamos o método numérico de Newton.

Geramos $w_{i}$ independentemente para as $n_{i}$ primeiras observações através da densidade (2.6) e para as $\left(n-n_{i}\right)$ observações restantes pela densidade (2.4), portanto a F.V. (2.3) dado a introdução da variável artificial $W$, pode ser escrita como em (2.5):

$$
L(\Theta \mid W, \mathbf{x})=\prod_{i=1}^{n}\left\{\sum_{j=1}^{J} P\left(j \mid \mathbf{x}, \gamma_{\mathbf{j}}\right) f_{j}\left(w_{i} \mid \mathbf{x}, \beta_{\mathbf{j}}\right)\right\}
$$

Podemos notar que, a introdução da variável artificial $W$ no modelo, 
elimina o cálculo do produto de integrais na F.V., mas continua ainda com produtos de mistura de distribuições, e uma alternativa seria transformar o modelo de mistura em um modelo de componentes independentes, introduzindo um outro grupo de variável artificial, $Z=\left[\mathbf{z}_{\mathbf{i 1}}, \ldots, \mathbf{z}_{\mathbf{i j}}\right], j=$ 1,$2 ; i=1, \ldots, n$, que segue uma distribuição multinomial (ver por exemplo, Diebolt e Robert, 1994), onde $z_{i j}=1$ se $w_{i}$ for considerado como originado de uma população com função densidade de probabilidade $f_{j} ;$ e $\sum_{j=1}^{J} \mathbf{z}_{\mathbf{i j}}=$ 1.

Com $J=2$ temos, $z_{i 1} \mid \Theta, W, \mathbf{x} \sim b\left(1, h_{i 1}\right) \quad$ (distribuição de Bernoulli), onde $\mathbf{z}_{\mathbf{i} 2}=1-\mathbf{z}_{\mathbf{i 1}}$, e $h_{i 1}$ é dado por:

$$
h_{i 1}=\frac{P\left(1 \mid \mathbf{x}, \gamma_{1}\right) f_{1}\left(w_{i} \mid \mathbf{x}, \beta_{1}\right)}{\sum_{j=1}^{2} P\left(j \mid \mathbf{x}, \gamma_{\mathbf{j}}\right) f_{j}\left(w_{i} \mid \mathbf{x}, \beta_{\mathbf{j}}\right)}
$$

e $h_{i 2}=1-h_{i 1}$.

Assim a densidade de $Z$ é dada por,

$$
\pi(Z) \propto h_{i 1}^{\mathbf{z}_{12}}\left(1-h_{i 1}\right)^{\mathbf{z}_{\mathbf{i 2}}}
$$

onde,

$$
\mathbf{z}_{\mathbf{i 1}}= \begin{cases}1 & \text { com probabilidade } h_{\mathrm{i} 1} \\ 0 & \text { com probabilidade }\left(1-h_{\mathbf{i} 1}\right)\end{cases}
$$

Observe que $\mathbf{z}_{\mathbf{i 1}}+\mathbf{z}_{\mathbf{i} 2}=1$, consequentemente temos, 


$$
\begin{aligned}
& \pi(Z) \propto \prod_{i=1}^{n}\left\{\left(\frac{P\left(1 \mid \mathbf{x}, \gamma_{1}\right) f_{1}\left(w_{i} \mid \mathbf{x}, \beta_{1}\right)}{\sum_{j=1}^{2} P\left(j \mid \mathbf{x}, \gamma_{\mathbf{j}}\right) f_{j}\left(w_{i} \mid \mathbf{x}, \beta_{\mathbf{j}}\right)}\right)^{\mathbf{z}_{\mathbf{i 1}}}\right. \\
& \left.\left(\frac{P\left(2 \mid \mathbf{x}, \gamma_{2}\right) f_{2}\left(w_{i} \mid \mathbf{x}, \beta_{2}\right)}{\sum_{j=1}^{2} P\left(j \mid \mathbf{x}, \gamma_{\mathbf{j}}\right) f_{j}\left(w_{i} \mid \mathbf{x}, \beta_{\mathbf{j}}\right)}\right)^{\mathrm{z}_{\mathrm{i2}}}\right\}
\end{aligned}
$$

resolvendo temos,

$$
\pi\left(\mathbf{z}_{1}, \ldots, \mathbf{z}_{\mathbf{n}}\right) \propto \frac{\prod_{i=1}^{n} \prod_{j=1}^{2}\left\{P\left(j \mid \mathbf{x}, \gamma_{\mathbf{j}}\right) f_{j}\left(w_{i} \mid \mathbf{x}, \beta_{\mathbf{j}}\right)\right\}^{\mathbf{z}_{\mathbf{j}}}}{\prod_{i=1}^{n}\left\{\sum_{j=1}^{2} P\left(j \mid \mathbf{x}, \gamma_{\mathbf{j}}\right) f_{j}\left(w_{i} \mid \mathbf{x}, \beta_{\mathbf{j}}\right)\right\}}
$$

Sendo $Z=\left[\mathbf{z}_{\mathbf{i 1}}, \mathbf{z}_{\mathbf{i} 2}\right]$ uma matriz de elementos independentes, a F.V. de $\Theta$, combinando a densidade (2.10) com a F.V. (2.5) pode ser escrita como:

$$
L(\Theta \mid W, Z, \mathbf{x})=\prod_{i=1}^{n} \prod_{j=1}^{2}\left\{P\left(j \mid \mathbf{x}, \gamma_{\mathbf{j}}\right) f_{j}\left(w_{i} \mid \mathbf{x}, \beta_{\mathbf{j}}\right)\right\}^{z_{\mathbf{j}}}
$$

onde, a variável artificial $W$ considera uma F.V. para dados iid e $Z$ converte o modelo de mistura em um modelo de componentes independentes. Com isso a complexidade do modelo é bastante simplificada.

Observar que a introdução desses dois grupos de variáveis artificiais é usado no método iterativo dado o valor dos parâmetros em cada iteração. 
Para cada iteração subsequente, devemos gerar novas variáveis artificiais ou latentes, como será feito usando métodos MCMC na análise Bayesiana do modelo proposto. 


\section{Capítulo 3}

\section{Modelos de Mistura para Dados sem Censuras}

\subsection{Modelo Bayesiano}

Para especificar um modelo Bayesiano, precisamos de dois componentes; a função de verossimilhança (F.V.) denotada por $\pi(\mathbf{t} \mid \Theta)$, que resume toda informação que os dados podem oferecer sobre os parâmetros; e a densidade à priori $\pi(\Theta)$, a qual indica um conhecimento prévio dos parâmetros. Quando o experimentador tem um conhecimento à priori dos parâmetros é atribuído ao modelo Bayesiano uma priori informativa, mas quando não existe essa informação à priori, consideramos uma priori não informativa (ver por exemplo, Box e Tiao, 1973).

Em resumo, o modelo Bayesiano é expresso pela densidade à posteriori $\pi(\Theta \mid \mathbf{t})$, de onde iremos obter resultados à posteriori de interesse. A densidade $\pi(\Theta \mid t)$ é dada à partir da fórmula de Bayes, onde combinamos $\pi(\mathbf{t} \mid \Theta) \operatorname{com} \pi(\Theta)$. 
- Fórmula de Bayes:

Suponha que $\mathrm{t}=\left(t_{1}, t_{2}, \ldots, t_{n}\right)$ é um vetor de $n$ observações onde a distribuição de probabilidade $\pi(\mathbf{t} \mid \Theta)$ depende dos valores dos $k$ parâmetros $\Theta=\left(\theta_{1}, \ldots, \theta_{k}\right)$, e $\Theta$ também tenha uma distribuição de probabilidade $\pi(\Theta)$, então,

$$
\pi(\mathbf{t} \mid \Theta) \pi(\Theta)=\pi(\mathbf{t}, \Theta)=\pi(\Theta \mid \mathbf{t}) \pi(\mathbf{t})
$$

Dado as observações, a distribuição condicional de $\Theta$ é dada por:

$$
\pi(\Theta \mid \mathbf{t})=\frac{\pi(\mathbf{t} \mid \Theta) \pi(\Theta)}{\pi(\mathbf{t})}
$$

onde:

$$
\pi(\mathbf{t})=\mathbb{E}(\pi(\mathbf{t} \mid \Theta))=\mathbf{c}^{-1}= \begin{cases}\int_{\Theta} \pi(\mathbf{t} \mid \Theta) \pi(\Theta) d \Theta & \Theta \text { contínuo } \\ \Sigma_{\Theta} \pi(\mathbf{t} \mid \Theta) \pi(\Theta) . & \Theta \text { discreto }\end{cases}
$$

$\mathbb{E}(\pi(\mathbf{t} \mid \Theta))$ é a esperança matemática de $\pi(\mathbf{t} \mid \Theta)$ em relação a densidade $\pi(\Theta)$, onde a soma ou a integral é calculada sobre a região de abrangência de $\Theta$.

Podemos então escrever (3.1) como,

$$
\pi(\Theta \mid \mathbf{t})=c L(\mathbf{t} \mid \Theta) \pi(\Theta)
$$


onde $c$ é a constante normalizadora necessária para que a integral ou a soma de $\pi(\Theta \mid \mathbf{t})$ dê 1 .

Portanto a densidade à posteriori (3.1) pode ser escrita por:

$$
\underbrace{\pi(\Theta \mid \mathbf{t})}_{\text {Posteriori }} \propto \underbrace{\pi(\Theta)}_{\text {Priori }} \underbrace{L(\mathbf{t} \mid \Theta)}_{\text {F.V. }}
$$

A partir da densidade (3.2) podemos fazer estimativas como por exemplo média, intervalo de credibilidade Bayesiano e previsões, para os parâmetros de interesse $\Theta=\left(\theta_{1}, \ldots, \theta_{k}\right)$.

Nem sempre conseguimos uma forma padrão fechada para calcular as densidades à posteriori marginais de $\Theta$, daí temos algumas alternativas como; métodos numéricos (ver por exemplo, Naylor e Smith, 1982); métodos de aproximação de integrais por Laplace (ver por exemplo, Achcar, 1989) ou algoritmos de simulação de amostras do tipo MCMC (ver por exemplo, Gelfand e Smith, 1990 ou Chib e Greenberg, 1994).

Diebolt e Robert (1994) consideram o uso do algoritmo amostrador de Gibbs para observações iid para o modelo de mistura (1.1) sem as covariáveis x. Kuo e Peng (1995) extenderam o uso desse algoritmo para o caso de dados censurados à direita e por intervalos, introduzindo as variáveis artificiais $Z$ e $W$ definidas no capítulo 2 . 


\subsection{Algoritmos de Simulação de Amostras}

Os métodos de simulação de amostras baseado na teoria de Cadeia de Markov com Monte Carlo (MCMC) atualmente tem sido bastante utilizados pelos estatísticos para simulações complexas, no caso de distribuições multivariadas não padronizadas.

O algoritmo amostrador de Gibbs é um dos métodos mais utilizados em inferência Bayesiana (ver por exemplo, Smith e Roberts, 1993; Tanner, 1993, ou Chib e Greenberg, 1993). Uma considerável antenção está agora sendo dada ao algoritmo Metropolis-Hastings (M-H), o qual foi introduzido por Metropolis et.al. (1953) e subsequentemente generalizado por Hastings (1970). Este algoritmo é extremamente versátil e considera o algoritmo amostrador de Gibbs como um caso especial (ver por exemplo, Gelman, 1992). O algoritmo Metropolis-Hastings já tinha sido usado extensivamente em física, mas apesar do artigo de Hastings (1970) este foi pouco conhecido pelos estatísticos até recentemente. Os artigos de Müller (1993) e Tierney (1994) mostram a importância deste algoritmo e estimula o interesse entre os estatísticos para o seu uso.

Para gerarmos amostras da distribuição conjunta à posteriori (3.2), utilizamos o algoritmo amostrador de Gibbs, considerando os valores iniciais, $\Theta^{(0)}=\left(\theta_{1}^{(0)}, \ldots, \theta_{k}^{(0)}\right)$ e seguindo os seguintes passos:

(i) Gerar amostras $W^{(1)}=\left(w_{1}^{(1)}, w_{2}^{(1)}, \ldots, w_{n}^{(1)}\right)$,

da densidade (2.4) quando os tempos de vida censurados à direita,

e da densidade (2.6) quando os tempos forem censurados por 
intervalos;

(ii) Gerar $\operatorname{amostras} Z^{(1)}=\left[\mathbf{z}_{\mathbf{i} 1}^{(1)}, \mathbf{z}_{\mathbf{i} 2}^{(\mathbf{1})}, \ldots, \mathbf{z}_{\mathbf{i j}}^{(1)}\right]$, da densidade (2.10), onde $Z^{(1)}=\left[\mathrm{z}_{\mathrm{i1}}^{(1)}, \mathrm{z}_{\mathrm{i2}}^{(1)}\right]$

(iii) Gerar uma amostra de $\Theta$, das distribuicões condicionais,

$$
\begin{aligned}
& \pi\left(\theta_{1} \mid \theta_{2}^{(0)}, \ldots, \theta_{p}^{(0)}, W^{(1)}, Z^{(1)}, \mathbf{x}\right), \pi\left(\theta_{2} \mid \theta_{1}^{(1)}, \theta_{3}^{(0)} \ldots, \theta_{p}^{(0)}, W^{(1)}, Z^{(1)}, \mathbf{x}\right), \\
& \ldots, \pi\left(\theta_{p} \mid \theta_{1}^{(1)}, \ldots, \theta_{p-1}^{(1)}, W^{(1)}, Z^{(1)}, \mathbf{x}\right) .
\end{aligned}
$$

Então continue as iterações repetindo os passos (i), (ii) e (iii) do algoritmo amostrador de Gibbs (3.3).

Quando as distribuições condicionais $\pi\left(\theta_{p} \mid \dot{\theta}_{1}^{(1)}, \ldots, \theta_{p-1}^{(1)}, W^{(1)}, Z^{(1)}, \mathbf{x}\right)$ não são padronizadas, uma alternativa é o uso do algoritmo MetropolisHastings, (ver Apêndice B).

\subsection{Critério de Convergência}

Para verificarmos se as amostras geradas estão realmente convergindo para uma distribuição estacionária, utilizamos um algoritmo baseado na técnica proposta por Gelman e Rubin (1992), onde iniciamos com pelo menos 2 cadeias paralelas com valores iniciais amostrados de uma distribuição bem comportada. Após as cadeias atingirem estacionariedade digamos na $n$-ésima iteração, consideramos as realizações $i$-ésima, $(i+h)$ ésima, ..., $(i+n h)$-ésima como uma amostra aleatória da distribuição 
desejada. Devemos assumir $h$ razoavelmente grande de tal forma que 2 valores sucessivos de $\theta_{k}$ sejam independentes (assim obtemos um amostra iid).

A convergência é então monitorada estimando o fator em que a escala da distribuição deva ser reduzida se as simulações são feitas até o limite quando o número de iterações tende ao infinito. Esse fator de redução de escala denotado em Gelman e Rubin (1992) por $\sqrt{\hat{R}}$ é apresentado no Apêndice $C$. Se esse fator for maior que 1 , devemos então considerar mais iterações, caso $\sqrt{\hat{R}}$ estiver próximo de 1 , a convergência está satisfeita.

\subsection{Mistura das Distribuições Potência Exponencial}

A motivação para o uso da distribuição potência exponencial no modelo de mistura (1.1) foi mencionada no capítulo 1 (seção 1.2). Consideramos então tempos de sobrevivência $\mathbf{t}$ (iid) (ver figura 5) associados à um único vetor de covariáveis $\mathbf{x}$ com diferentes níveis, que podem estar influenciando esses tempos e as probabilidades de incidência; uma mistura de $J=2$ distribuições potência exponencial com densidade (1.4) no modelo (1.1), $\operatorname{com} \theta_{j}=\left(\alpha_{j}+\beta_{j} x_{i k}\right), j=1,2, k=1,2, \ldots, K, K$ é o número de níveis de $\mathbf{x}$; e o modelo de regressão logística (1.2) para explicar as probabilidades de incidência da $j$-ésima população dado por:

$$
\begin{aligned}
& P(1 \mid \mathbf{x}, \gamma, \tau)=\frac{e^{\gamma+\tau x_{i k}}}{1+e^{\gamma+\tau x_{i k}}}, \\
& P(2 \mid \mathbf{x}, \gamma, \tau)=1-P(1 \mid \mathbf{x}, \gamma, \tau)=\frac{1}{1+e^{\gamma+\tau x_{i k}}} .
\end{aligned}
$$


Assumindo independência à priori entre os parâmetros, consideramos as seguintes densidades à priori para $\alpha_{1}, \beta_{1}, \sigma_{1}, \delta_{1}, \alpha_{2}, \beta_{2}, \sigma_{2}, \delta_{2}, \gamma$, e $\tau$ :

(i) $\quad \alpha_{1} \sim N\left(\alpha_{10}, \sigma_{11}^{2}\right) ; \alpha_{10}, \sigma_{11}^{2}$ conhecidos,

(ii) $\quad \beta_{1} \sim N\left(\beta_{10}, \sigma_{12}^{2}\right) ; \beta_{10}, \sigma_{12}^{2}$ conhecidos,

(iii) $\quad \sigma_{1} \sim \Gamma\left[m_{11}, n_{11}\right] ; m_{11}, n_{11}$ conhecidos,

(iv) $\delta_{1} \sim N\left(\delta_{10}, \sigma_{13}^{2}\right) ; \delta_{10}, \sigma_{13}^{2}$ conhecidos,

(v) $\quad \alpha_{2} \sim N\left(\alpha_{20}, \sigma_{21}^{2}\right) ; \alpha_{20}, \sigma_{21}^{2}$ conhecidos,

(vi) $\beta_{2} \sim N\left(\beta_{20}, \sigma_{22}^{2}\right) ; \beta_{20}, \sigma_{22}^{2}$ conhecidos,

(vii) $\sigma_{2} \sim \Gamma\left[m_{22}, n_{22}\right] ; m_{22}, n_{22}$ conhecidos,

(viii) $\delta_{2} \sim N\left(\delta_{20}, \sigma_{23}^{2}\right) ; \delta_{20}, \sigma_{23}^{2}$ conhecidos,

(ix) $\quad \gamma \sim N\left(\gamma_{0}, \sigma_{14}^{2}\right) ; \gamma_{0}, \sigma_{14}^{2}$ conhecidos,

(x) $\quad \tau \sim N\left(\tau_{0}, \sigma_{24}^{2}\right) ; \tau_{0}, \sigma_{24}^{2}$ conhecidos,

onde $N\left(\mu, \sigma^{2}\right)$ denota uma distribuição normal com média $\mu$ e variância $\sigma^{2}$; e $\Gamma[a, b]$ denota uma distribuição gamma com média $a / b$ e variância $a / b^{2}$.

Assim, a densidade conjunta à priori para $\Theta$ é, 


$$
\begin{aligned}
& \pi(\Theta) \propto \exp \left\{-\frac{1}{2 \sigma_{11}^{2}}\left(\alpha_{1}-\alpha_{10}\right)^{2}\right\} \quad \exp \left\{-\frac{1}{2 \sigma_{12}^{2}}\left(\beta_{1}-\beta_{10}\right)^{2}\right\} \\
& \exp \left\{-\frac{1}{2 \sigma_{13}^{2}}\left(\delta_{1}-\delta_{10}\right)^{2}\right\} \exp \left\{-\frac{1}{2 \sigma_{14}^{2}}\left(\gamma-\gamma_{0}\right)^{2}\right\} \\
& \exp \left\{-\frac{1}{2 \sigma_{24}^{2}}\left(\tau-\tau_{0}\right)^{2}\right\} \quad \sigma_{1}^{m_{11}-1} \sigma_{2}^{m_{22}-1} \exp \left\{-n_{11} \sigma_{1}-n_{22} \sigma_{2}\right\} \\
& \exp \left\{-\frac{1}{2 \sigma_{21}^{2}}\left(\alpha_{2}-\alpha_{20}\right)^{2}\right\} \exp \left\{-\frac{1}{2 \sigma_{22}^{2}}\left(\beta_{2}-\beta_{20}\right)^{2}\right\} \\
& \exp \left\{-\frac{1}{2 \sigma_{23}^{2}}\left(\delta_{2}-\delta_{20}\right)^{2}\right\}
\end{aligned}
$$

e $L(\Theta \mid \mathbf{t}, \mathbf{x})$ definido em (2.1) é dado por:

$$
\begin{aligned}
L(\Theta \mid \mathbf{t}, \mathbf{x})= & \prod_{k=1}^{K} \prod_{i=1}^{n}\left\{( \frac { e ^ { \gamma + \tau x _ { i k } } } { 1 + e ^ { \gamma + \tau x _ { i k } } } ) \left[w\left(\delta_{1}\right) \sigma_{1}^{-1}\right.\right. \\
& \left.\exp \left(-c\left(\delta_{1}\right)\left|\frac{t_{i k}-\left(\alpha_{1}+\beta_{1} x_{i k}\right)}{\sigma_{1}}\right|^{2 /\left(1+\delta_{1}\right)}\right)\right]+\left(\frac{1}{\left(1+e^{\left.\gamma+\tau x_{i k}\right)}\right)}\right. \\
& {\left.\left[w\left(\delta_{2}\right) \sigma_{2}^{-1} \exp \left(-c\left(\delta_{2}\right)\left|\frac{t_{i k}-\left(\alpha_{2}+\beta_{2} x_{i k}\right)}{\sigma_{2}}\right|^{2 /\left(1+\delta_{2}\right)}\right)\right]\right\} }
\end{aligned}
$$

Portanto a densidade à posteriori conjunta para $\Theta$, é dada por: 


$$
\begin{aligned}
\pi(\Theta \mid \mathbf{t}, \mathbf{x}) \propto & \pi(\Theta) \prod_{k=1}^{K} \prod_{i=1}^{n}\left\{( \frac { e ^ { \gamma + \tau x _ { i k } } } { 1 + e ^ { \gamma + \tau x _ { i k } } } ) \left[w\left(\delta_{1}\right) \sigma_{1}^{-1}\right.\right. \\
& \left.\exp \left(-c\left(\delta_{1}\right)\left|\frac{t_{i k}-\left(\alpha_{1}+\beta_{1} x_{i k}\right)}{\sigma_{1}}\right|^{2 /\left(1+\delta_{1}\right)}\right)\right]+\left(\frac{1}{\left(1+e^{\gamma+\tau x_{i k}}\right)}\right) \\
& {\left.\left[w\left(\delta_{2}\right) \sigma_{2}^{-1} \exp \left(-c\left(\delta_{2}\right)\left|\frac{t_{i k}-\left(\alpha_{2}+\beta_{2} x_{i k}\right)}{\sigma_{2}}\right|^{2 /\left(1+\delta_{2}\right)}\right)\right]\right\} }
\end{aligned}
$$

Para tentar simplificar a implementação do algoritmo amostrador de Gibbs, introduzimos a variável artificial $Z$ com densidade (2.10) definida no capítulo 2. Assim, a densidade conjunta à posteriori de $\Theta(3.8)$ combinando a densidade (2.10) com a F.V. (3.7) é dada por:

$$
\pi(\Theta \mid \mathbf{t}, Z, \mathbf{x}) \propto \pi(\Theta)\left\{\prod_{k=1}^{K} \prod_{i=1}^{n} \prod_{j=1}^{2}\left\{P\left(j \mid x_{i k}, \gamma_{\mathbf{j}}\right) f_{j}\left(t_{i k} \mid x_{i k}, \beta_{\mathbf{j}}\right)\right\}^{z_{i k j}}\right\}
$$

que pode ser escrita como,

$$
\begin{aligned}
& \pi(\Theta \mid \mathbf{t}, Z, \mathbf{x}) \propto \pi(\Theta)\left\{\frac{\exp \left(r \gamma+a_{1} \tau\right)}{\prod_{k=1}^{K} \prod_{i=1}^{n}\left(1+e^{\gamma+\tau x_{i k}}\right)}\right\} \frac{\left[w ( \delta _ { 1 } ] ^ { r } \left[w\left(\delta_{2}\right]^{(n-r)}\right.\right.}{\sigma_{1}^{r} \sigma_{2}^{(n-r)}} \\
& \exp \left\{-\frac{c\left(\delta_{1}\right)}{\sigma_{1}^{2 /\left(1+\delta_{1}\right)}} B_{1}\left(\alpha_{1}, \beta_{1}, \delta_{1}\right)-\frac{c\left(\delta_{2}\right)}{\sigma_{2}^{2 /\left(1+\delta_{2}\right)}} B_{2}\left(\alpha_{2}, \beta_{2}, \delta_{2}\right)\right\}
\end{aligned}
$$

onde: 
$\pi(\Theta)$ é dado em $(3.6) ; \cdot r=\sum_{k=1}^{K} \sum_{i=1}^{n} z_{i k 1}$

$(n-r)=\sum_{k=1}^{K} \sum_{i=1}^{n} z_{i k 2}, a_{1}=\sum_{k=1}^{K} \sum_{i=1}^{n} x_{i k} z_{i k 1}$

$B_{1}\left(\alpha_{1}, \beta_{1}, \delta_{1}\right)=\sum_{k=1}^{K} \sum_{i=1}^{n} z_{i k 1}\left|t_{i k}-\left(\alpha_{1}+\beta_{1} x_{i k}\right)\right|^{2 /\left(1+\delta_{1}\right)}$

$B_{2}\left(\alpha_{2}, \beta_{2}, \delta_{2}\right)=\sum_{k=1}^{K} \sum_{i=1}^{n} z_{i k 2}\left|t_{i k}-\left(\alpha_{2}+\beta_{2} x_{i k}\right)\right|^{2 /\left(1+\delta_{2}\right)}$

Para gerarmos amostras da densidade conjunta (3.10), seguimos os passos (ii) e (iii) do algoritmo de Gibbs (3.3), onde as distribuições condicionais marginais para $\alpha_{1}, \beta_{1}, \sigma_{1}, \delta_{1}, \alpha_{2}, \beta_{2}, \sigma_{2}, \delta_{2}, \gamma$, e $\tau$, são dadas por:

(i) $\quad \pi\left(\alpha_{1} \mid \beta_{1}, \sigma_{1}, \delta_{1}, \alpha_{2}, \beta_{2}, \sigma_{2}, \delta_{2}, \gamma, \tau, Z, \mathbf{t}, \mathbf{x}\right) \propto$

$$
\exp \left\{-\frac{1}{2 \sigma_{11}^{2}}\left(\alpha_{1}-\alpha_{10}\right)^{2}\right\} \Psi_{1}(\Theta)
$$

onde,

$$
\Psi_{1}(\Theta)=\exp \left\{-\frac{c\left(\delta_{1}\right)}{\sigma_{1}^{2 /\left(1+\delta_{1}\right)}} B_{1}\left(\alpha_{1}, \beta_{1}, \delta_{1}\right)\right\}
$$

$$
\begin{aligned}
& \pi\left(\beta_{1} \mid \alpha_{1}, \sigma_{1}, \delta_{1}, \alpha_{2}, \beta_{2}, \sigma_{2}, \delta_{2}, \gamma, \tau, Z, \mathbf{t}, \mathbf{x}\right) \propto \\
& \exp \left\{-\frac{1}{2 \sigma_{12}^{2}}\left(\beta_{1}-\beta_{10}\right)^{2}\right\} \Psi_{2}(\Theta)
\end{aligned}
$$

onde,

$$
\Psi_{2}(\Theta)=\exp \left\{-\frac{c\left(\delta_{1}\right)}{\sigma_{1}^{2 /\left(1+\delta_{1}\right)}} B_{1}\left(\alpha_{1}, \beta_{1}, \delta_{1}\right)\right\}
$$


(iii)

$$
\begin{aligned}
& \pi\left(\sigma_{1} \mid \alpha_{1}, \beta_{1}, \delta_{1}, \alpha_{2}, \beta_{2}, \sigma_{2}, \delta_{2}, \gamma, \tau, Z, \mathbf{t}, \mathbf{x}\right) \propto . \\
& \sigma_{1}^{m_{11}-1} \exp \left\{-n_{11} \sigma_{1}\right\} \Psi_{3}(\Theta)
\end{aligned}
$$

onde,

$$
\Psi_{3}(\Theta)=\exp \left\{-r \ln \left(\sigma_{1}\right)-\sigma_{1}^{-2 /\left(1+\delta_{1}\right)} c\left(\delta_{1}\right) B_{1}\left(\alpha_{1}, \beta_{1}, \delta_{1}\right)\right\}
$$

(iv)

$$
\begin{aligned}
& \pi\left(\delta_{1} \mid \alpha_{1}, \beta_{1}, \sigma_{1}, \alpha_{2}, \beta_{2}, \sigma_{2}, \delta_{2}, \gamma, \tau, Z, \mathbf{t}, \mathbf{x}\right) \propto \\
& \exp \left\{-\frac{1}{2 \sigma_{13}^{2}}\left(\delta_{1}-\delta_{10}\right)^{2}\right\} \Psi_{4}(\Theta)
\end{aligned}
$$

onde,

$$
\Psi_{4}(\Theta)=\exp \left\{r \ln \left(w\left(\delta_{1}\right)\right)-\frac{c\left(\delta_{1}\right)}{\sigma_{1}^{2 /\left(1+\delta_{1}\right)}} B_{1}\left(\alpha_{1}, \beta_{1}, \delta_{1}\right)\right\}
$$

(v) $\quad \pi\left(\alpha_{2} \mid \alpha_{1}, \beta_{1}, \sigma_{1}, \delta_{1}, \beta_{2}, \sigma_{2}, \delta_{2}, \gamma, \tau, Z, \mathbf{t}, \mathbf{x}\right) \propto$

$$
\exp \left\{-\frac{1}{2 \sigma_{21}^{2}}\left(\alpha_{2}-\alpha_{20}\right)^{2}\right\} \Psi_{5}(\Theta)
$$

onde,

$$
\Psi_{5}(\Theta)=\exp \left\{-\frac{c\left(\delta_{2}\right)}{\sigma_{2}^{2 /\left(1+\delta_{2}\right)}} B_{2}\left(\alpha_{2}, \beta_{2}, \delta_{2}\right)\right\}
$$

(vi) $\quad \pi\left(\beta_{2} \mid \alpha_{1}, \beta_{1}, \sigma_{1}, \delta_{1}, \alpha_{2}, \sigma_{2}, \delta_{2}, \gamma, \tau, Z, \mathbf{t}, \mathbf{x}\right) \propto$

$$
\exp \left\{-\frac{1}{2 \sigma_{22}^{2}}\left(\beta_{2}-\beta_{20}\right)^{2}\right\} \Psi_{6}(\Theta)
$$

onde, 


$$
\Psi_{6}(\Theta)=\exp \left\{-\frac{c\left(\delta_{2}\right)}{\sigma_{2}^{2 /\left(1+\delta_{2}\right)}} B_{2}\left(\alpha_{2}, \beta_{2}, \delta_{2}\right)\right\}
$$

(vii)

$$
\begin{aligned}
& \pi\left(\sigma_{2} \mid \alpha_{1}, \beta_{1}, \delta_{1}, \sigma_{1}, \alpha_{2}, \beta_{2}, \delta_{2}, \gamma, \tau, Z, \mathbf{t}, \mathbf{x}\right) \propto \\
& \sigma_{2}^{m_{22}-1} \exp \left\{-n_{22} \sigma_{2}\right\} \Psi_{7}(\Theta)
\end{aligned}
$$

onde,

$$
\Psi_{7}(\Theta)=\exp \left\{-(n-r) \ln \left(\sigma_{2}\right)-\sigma_{2}^{-2 /\left(1+\delta_{2}\right)} c\left(\delta_{2}\right) B_{2}\left(\alpha_{2}, \beta_{2}, \delta_{2}\right)\right\}
$$

(viii) $\quad \pi\left(\delta_{2} \mid \alpha_{1}, \beta_{1}, \sigma_{1}, \delta_{1}, \alpha_{2}, \beta_{2}, \sigma_{2}, \gamma, \tau, Z, \mathbf{t}, \mathbf{x}\right) \propto$

$$
\exp \left\{-\frac{1}{2 \sigma_{23}^{2}}\left(\delta_{2}-\delta_{20}\right)^{2}\right\} \Psi_{8}(\Theta)
$$

onde,

$$
\Psi_{8}(\Theta)=\exp \left\{(n-r) \ln \left(w\left(\delta_{2}\right)\right)-\frac{c\left(\delta_{2}\right)}{\sigma_{2}^{2 /\left(1+\delta_{2}\right)}} B_{2}\left(\alpha_{2}, \beta_{2}, \delta_{2}\right)\right\}
$$

(ix)

$$
\begin{aligned}
& \pi\left(\gamma \mid \alpha_{1}, \beta_{1}, \sigma_{1}, \delta_{1}, \alpha_{2}, \beta_{2}, \sigma_{2}, \delta_{2}, \tau, Z, \mathbf{t}, \mathbf{x}\right) \propto \\
& \exp \left\{-\frac{1}{2 \sigma_{14}^{2}}\left(\gamma-\gamma_{0}\right)^{2}\right\} \Psi_{9}(\Theta)
\end{aligned}
$$

onde,

$$
\Psi_{9}(\Theta)=\exp \left\{r \gamma-\sum_{k=1}^{K} \sum_{i=1}^{n} \ln \left(1+e^{\gamma+\tau x_{i k}}\right)\right\}
$$

(x)

$$
\begin{aligned}
& \pi\left(\tau \mid \alpha_{1}, \beta_{1}, \sigma_{1}, \delta_{1}, \alpha_{2}, \beta_{2}, \sigma_{2}, \delta_{2}, \gamma, Z, \mathbf{t}, \mathbf{x}\right) \propto \\
& \exp \left\{-\frac{1}{2 \sigma_{24}^{2}}\left(\tau-\tau_{0}\right)^{2}\right\} \Psi_{10}(\Theta)
\end{aligned}
$$


onde,

$$
\Psi_{10}(\Theta)=\exp \left\{a_{1} \tau-\sum_{k=1}^{K} \sum_{i=1}^{n} \ln \left(1+e^{\gamma+r x_{i k}}\right)\right\}
$$

Observe que as densidades (3.11) não tem uma forma padrão conhecida, portanto devemos usar o algoritmo Metropolis-Hastings para gerar todos os parâmetros de $\Theta$.

\subsection{Mistura das Distribuições Normal-Exponencial}

Consideramos no modelo de mistura (1.1) as probabilidades de incidência (3.4) com uma especificação proporcional padrão para as distribuições de latências condionais, com densidades,

$$
\begin{aligned}
& f_{1}\left(t_{i k} \mid x_{i k}, \beta_{1}\right)=\frac{1}{\sqrt{2 \pi} \sigma} \exp \left\{-\frac{1}{2 \sigma^{2}}\left(t_{i k}-\left(\alpha_{1}+\beta_{1} x_{i k}\right)\right)^{2}\right\} \\
& f_{2}\left(t_{i k} \mid x_{i k}, \beta_{2}\right)=\lambda_{i k} \exp \left\{-\lambda_{i k} t_{i}\right\}
\end{aligned}
$$

onde:

$$
\begin{gathered}
\lambda_{i k}=\left(\alpha_{2}+\beta_{2} x_{i k}\right)^{-1}>0 ; \beta_{1}=\left(\alpha_{1}, \beta_{1}, \sigma\right) ; \beta_{2}=\left(\alpha_{2}, \beta_{2}\right) \\
-\infty<t_{i k}<+\infty ; \sigma^{2}>0 ;-\infty<\left(\alpha_{1}+\beta_{2} x_{i k}\right)<+\infty
\end{gathered}
$$

Assumindo independência à priori entre os parâmetros, consideramos as seguintes densidades à priori para $\alpha_{1}, \beta_{1}, \sigma, \alpha_{2}, \beta_{2}, \gamma, \tau$ :

(i) $\alpha_{1}, \beta_{1}, \sigma, \alpha_{2}, \beta_{2}$ localmente uniformes, 
(ii) $\gamma \sim N\left(\gamma_{0}, s_{1}^{2}\right), \gamma_{0}, s_{1}^{2}$ conhecidos,

(iii) $\tau \sim N\left(\tau_{0}, s_{2}^{2}\right), \tau_{0}, s_{2}^{2}$ conhecidos

onde $N(a, b)$ denota uma distribuição normal com média $a$ e variância $b$.

Considerando a introdução da variável artificial $Z$, a densidade à posteriori conjunta de $\Theta=\left(\alpha_{1}, \beta_{1}, \sigma, \alpha_{2}, \beta_{2}, \gamma, \tau\right)$ é dada por:

$$
\begin{aligned}
& \pi(\Theta \mid \mathbf{t}, Z, \mathbf{x}) \propto \frac{\left\{\prod_{k=1}^{K} \prod_{i=1}^{n}\left(\alpha_{2}+\beta_{2} x_{i k}\right)^{-z_{i k 2}}\right\}}{\left\{\prod_{k=1}^{K} \prod_{i=1}^{n}\left(1+e^{\gamma+r x_{i k}}\right)\right\}} \frac{e^{\gamma r+\tau a_{1}}}{\sigma^{\tau}} \\
& \exp \left\{-\frac{1}{2 s_{1}^{2}}\left(\gamma-\gamma_{0}\right)^{2}-\frac{1}{2 s_{2}^{2}}\left(\tau-\tau_{0}\right)^{2}\right\} \\
& \left\{\operatorname { e x p } \left\{-\frac{1}{2 \sigma^{2}} \sum_{k=1}^{K} \sum_{i=1}^{n} z_{i k 1}\left(t_{i k}-\left(\alpha_{1}+\beta_{1} x_{i k}\right)\right)^{2}-\right.\right. \\
& \left.\left.\sum_{k=1}^{K} \sum_{i=1}^{n} z_{i k 2} t_{i k}\left(\alpha_{2}+\beta_{2} x_{i k}\right)^{-1}\right\}\right\}
\end{aligned}
$$

onde $r=\sum_{k=1}^{K} \sum_{i=1}^{n} z_{i k 1}$

$(n-r)=\sum_{k=1}^{K} \sum_{i=1}^{n} z_{i k 2}$ e $a_{1}=\sum_{k=1}^{K} \sum_{i=1}^{n} x_{i k} z_{i k 1}$.

Geramos amostras da densidade à posteriori conjunta (3.14), usando os passos (ii) e (iii) do algoritmo de Gibbs (3.3), onde as distribuições condicionais para os parâmetros são dadas por: 
(i) $\pi\left(v \mid \alpha_{1}, \beta_{1}, \alpha_{2}, \beta_{2}, \gamma, \tau, Z, \mathbf{t}, \mathbf{x}\right) \sim$

$$
\Gamma\left(\frac{r}{2}+1, \frac{\sum_{k=1}^{K} \sum_{i=1}^{n} z_{i k 1}\left(t_{i k}-\left(\alpha_{1}+\beta_{1} x_{i k}\right)\right)^{2}}{2}\right)
$$

onde $v=\sigma^{-2}$

(ii)

$$
\begin{aligned}
& \pi\left(\alpha_{1} \mid \beta_{1}, \sigma, \alpha_{2}, \beta_{2}, \gamma, \tau, Z, \mathbf{t}, \mathbf{x}\right) \sim \\
& N\left(\frac{\sum_{k=1}^{K} \sum_{i=1}^{n} z_{i k 1}\left(t_{i k}-\beta_{1} x_{i k}\right)}{r}, \frac{\sigma^{2}}{r}\right)
\end{aligned}
$$

(iii) $\quad \pi\left(\beta_{1} \mid \alpha_{1}, \sigma, \alpha_{2}, \beta_{2}, \gamma, \tau, Z, \mathbf{t}, \mathbf{x}\right) \sim$

$$
N\left(\frac{\sum_{k=1}^{K} \sum_{i=1}^{n} z_{i k 1} x_{i k}\left(t_{i k}-\alpha_{1}\right)}{\sum_{k=1}^{K} \sum_{i=1}^{n} z_{i k 1} x_{i k}^{2}}, \frac{\sigma^{2}}{\sum_{k=1}^{K} \sum_{i=1}^{n} z_{i k 1} x_{i k}^{2}}\right)
$$

(iv) $\quad \pi\left(\alpha_{2} \mid \alpha_{1}, \beta_{1}, \sigma, \beta_{2}, \gamma, \tau, Z, \mathbf{t}, \mathbf{x}\right) \propto \alpha_{2}^{-(n-r)}$

$$
\exp \left\{-\frac{1}{\alpha_{2}} \sum_{k=1}^{K} \sum_{i=1}^{n} z_{i k 2} t_{i k}\left(1+\frac{\beta_{2}}{\alpha_{2}} x_{i k}\right)^{-1}\right\} \Psi_{1}(\Theta)
$$

onde $\Psi_{1}(\Theta)=\prod_{k=1}^{K} \prod_{i=1}^{n}\left(1+\frac{\beta_{2}}{\alpha_{2}} x_{i k}\right)^{-z_{i k 2}}$,

(v) $\quad \pi\left(\beta_{2} \mid \alpha_{1}, \beta_{1}, \sigma, \alpha_{2}, \gamma, \tau, Z, \mathbf{t}, \mathbf{x}\right) \propto \beta_{2}^{-(n-r)}$

$$
\exp \left\{-\frac{1}{\beta_{2}} \sum_{k=1}^{K} \sum_{i=1}^{n} z_{i k 2} t_{i k}\left(\frac{\alpha_{2}}{\beta_{2}}+x_{i k}\right)^{-1}\right\} \Psi_{2}(\Theta)
$$


onde $\Psi_{2}(\Theta)=\prod_{k=1}^{K} \prod_{i=1}^{n}\left(\frac{\alpha_{2}}{\beta_{2}}+x_{i k}\right)^{-z_{i k 2}}$

$$
\pi\left(\gamma \mid \alpha_{1}, \beta_{1}, \sigma, \alpha_{2}, \beta_{2}, \tau, Z, \mathbf{t}, \mathbf{x}\right) \propto \exp \left\{-\frac{1}{2 s_{1}^{2}}\left(\gamma-\gamma_{0}\right)^{2}\right\} \Psi_{3}(\Theta)
$$

onde $\Psi_{3}(\Theta)=\exp \left\{\gamma r-\sum_{k=1}^{K} \sum_{i=1}^{n} \ln \left(1+e^{\gamma+\tau x_{i k}}\right)\right\}$

(vii) $\left.\quad \pi\left(\tau \mid \alpha_{1}, \beta_{1}, \sigma, \alpha_{2}, \beta_{2}, \gamma, Z, \mathbf{t}, \mathbf{x}\right) \propto \exp \left\{-\frac{1}{2 s_{2}^{2}}\left(\tau-\tau_{0}\right)^{2}\right)\right\} \Psi_{4}(\Theta)$ onde $\Psi_{4}(\Theta)=\exp \left\{\tau a_{1}-\sum_{k=1}^{K} \sum_{i=1}^{n} \ln \left(1+e^{\gamma+\tau x_{i k}}\right)\right\}$

Aqui, $\Gamma(a, b)$ denota uma distribuição gamma com média $a / b$ e variância $a / b^{2}$.

Como podemos observar, precisamos usar o algoritmo MetropolisHastings para gerar as variáveis $\alpha_{2}, \beta_{2}, \gamma$ e $\tau$. Onde geramos candidatos para $\alpha_{2}$ e $\beta_{2}$ de uma distribuição gamma inversa; e para as variáveis $\gamma$ e $\tau$ das distribuições normais $N\left(\gamma_{0}, s_{1}^{2}\right)$ e $N\left(\tau_{0}, s_{2}^{2}\right)$, respectivamente.

\subsection{Mistura de duas Distribuições Normais}

Consideramos agora, uma mistura de duas distribuições normais no modelo (1.1), com densidades, 


$$
\begin{aligned}
& f_{1}\left(t_{i k} \mid x_{i k}, \beta_{1}\right)=\frac{1}{\sqrt{2 \pi} \sigma_{1}} \exp \left\{-\frac{1}{2 \sigma_{1}^{2}}\left(t_{i k}-\left(\alpha_{1}+\beta_{1} x_{i k}\right)\right)^{2}\right\} \\
& f_{2}\left(t_{i k} \mid x_{i k}, \beta_{2}\right)=\frac{1}{\sqrt{2 \pi} \sigma_{2}} \exp \left\{-\frac{1}{2 \sigma_{2}^{2}}\left(t_{i k}-\left(\alpha_{2}-\beta_{2} x_{i k}\right)\right)^{2}\right\},
\end{aligned}
$$

onde: $\beta_{1}=\left(\alpha_{1}, \beta_{1}, \sigma_{1}\right) ; \beta_{2}=\left(\alpha_{2}, \beta_{2}, \sigma_{2}\right) ; \sigma_{j}>0$;

$$
-\infty<\left(\alpha_{j}+\beta_{j} x_{i k}\right)<+\infty \text {; e }-\infty<t_{i k}<+\infty ; j=1,2 .
$$

Para modelar as probabilidades de incidência consideramos o mesmo modelo logístico (3.4); e assumindo indepedência à priori entre os parâmetros atribuímos as seguintes densidades à priori para $\alpha_{1}, \beta_{1}, \sigma_{1}, \alpha_{2}, \beta_{2}, \sigma_{2}, \gamma$ e $\tau$ :

(i) $\alpha_{1}, \beta_{1}, \sigma_{1}, \alpha_{2}, \beta_{2}, \sigma_{2}$ localmente uniforme,

(ii) $\gamma \sim N\left(\gamma_{0}, s_{1}^{2}\right), \gamma_{0}, s_{1}^{2}$ conhecidos,

(iii) $\quad \tau \sim N\left(\gamma_{0}, s_{2}^{2}\right), \tau_{0}, s_{2}^{2}$ conhecidos,

A densidade à posteriori conjunta para $\Theta=\left(\alpha_{1}, \beta_{1}, \sigma_{1}, \alpha_{2}, \beta_{2}, \sigma_{2}, \gamma, \tau\right)$, considerando a variável artificial $Z$, é dada por: 


$$
\begin{aligned}
& \pi(\Theta \mid \mathbf{t}, Z, \mathbf{x}) \propto \frac{\sigma_{1}^{-r} \sigma_{2}^{-(n-r)}}{\left\{\prod_{k=1}^{K} \prod_{i=1}^{n}\left(1+e^{\gamma+\tau x_{i k}}\right)\right\}} \\
& \exp \left\{-\frac{1}{2 s_{1}^{2}}\left(\gamma-\gamma_{0}\right)^{2}+\gamma r-\frac{1}{2 s_{2}^{2}}\left(\tau-\tau_{0}\right)^{2}+\tau a_{1}\right\} \\
& \left\{\operatorname { e x p } \left\{-\frac{1}{2 \sigma_{1}^{2}} \sum_{k=1}^{K} \sum_{i=1}^{n} z_{i k 1}\left(t_{i k}-\left(\alpha_{1}+\beta_{1} x_{i k}\right)\right)^{2}-\right.\right. \\
& \left.\left.\frac{1}{2 \sigma_{2}^{2}} \sum_{k=1}^{K} \sum_{i=1}^{n} z_{i k 2}\left(t_{i k}-\left(\alpha_{2}+\beta_{2} x_{i k}\right)\right)^{2}\right\}\right\}
\end{aligned}
$$

onde $r,(n-r)$ e $a_{1}$ são definidos em (3.14).

Portanto as distribuições condicionais para o algoritmo Gibbs são dadas por:

(i)

$$
\begin{aligned}
& \pi\left(v \mid \alpha_{1}, \beta_{1}, \alpha_{2}, \beta_{2}, \sigma_{2}, \gamma, \tau, Z, \mathbf{t}, \mathbf{x}\right) \sim \\
& \Gamma\left(\frac{r}{2}+1, \frac{\sum_{k=1}^{K} \sum_{i=1}^{n} z_{i k 1}\left(t_{i k}-\left(\alpha_{1}+\beta_{1} x_{i k}\right)\right)^{2}}{2}\right)
\end{aligned}
$$

onde $v=\sigma_{1}^{-2}$

(ii)

$$
\begin{aligned}
& \pi\left(\alpha_{1}, \mid \beta_{1}, \sigma_{1}, \alpha_{2}, \beta_{2}, \sigma_{2}, \gamma, \tau, Z, \mathbf{t}, \mathbf{x}\right) \sim \\
& N\left(\frac{\sum_{k=1}^{K} \sum_{i=1}^{n} z_{i k 1}\left(t_{i k}-\beta_{1} x_{i k}\right)}{r}, \frac{\sigma_{1}^{2}}{r}\right)
\end{aligned}
$$


(iii)

$$
\begin{aligned}
& \pi\left(\beta_{1} \mid \alpha_{1}, \sigma_{1}, \alpha_{2}, \beta_{2}, \sigma_{2}, \gamma, \tau, Z, \mathbf{t}, \mathbf{x}\right) \sim \\
& N\left(\frac{\sum_{k=1}^{K} \sum_{i=1}^{n} z_{i k 1} x_{i k}\left(t_{i k}-\alpha_{1}\right)}{\sum_{k=1}^{K} \sum_{i=1}^{n} z_{i k 1} x_{i k}^{2}}, \frac{\sigma_{1}^{2}}{\sum_{k=1}^{K} \sum_{i=1}^{n} z_{i k 1} x_{i k}^{2}}\right)
\end{aligned}
$$

(iv)

$$
\begin{aligned}
& \pi\left(u \mid \alpha_{1}, \beta_{1}, \sigma_{1}, \alpha_{2}, \beta_{2}, \gamma, \tau, Z, \mathbf{t}, \mathbf{x}\right) \sim \\
& \Gamma\left(\frac{(n-r)}{2}+1, \frac{\sum_{k=1}^{K} \sum_{i=1}^{n} z_{i k 2}\left(t_{i k}-\left(\alpha_{2}+\beta_{2} x_{i k}\right)\right)^{2}}{2}\right)
\end{aligned}
$$

onde $u=\sigma_{2}^{-2}$

(v)

$$
\pi\left(\alpha_{2} \mid \alpha_{1}, \beta_{1}, \sigma_{1}, \beta_{2}, \sigma_{2}, \gamma, \tau, Z, \mathbf{t}, \mathbf{x}\right) \sim
$$

$$
N\left(\frac{\sum_{k=1}^{K} \sum_{i=1}^{n} z_{i k 2}\left(t_{i k}-\beta_{2} x_{i k}\right)}{(n-r)}, \frac{\sigma_{2}^{2}}{(n-r)}\right)
$$

(vi) $\quad \pi\left(\beta_{2} \mid \alpha_{1}, \beta_{1}, \sigma_{1}, \alpha_{2}, \sigma_{2}, \gamma, \tau, Z, \mathbf{t}, \mathbf{x}\right) \sim$

$$
N\left(\frac{\sum_{k=1}^{K} \sum_{i=1}^{n} z_{i k 2} x_{i k}\left(t_{i k}-\alpha_{2}\right)}{\sum_{k=1}^{K} \sum_{i=1}^{n} z_{i k 2} x_{i k}^{2}}, \frac{\sigma_{2}^{2}}{\sum_{k=1}^{K} \sum_{i=1}^{n} z_{i k 2} x_{i k}^{2}}\right)
$$

(vii) $\quad \pi\left(\gamma \mid \alpha_{1}, \beta_{1}, \sigma_{1}, \alpha_{2}, \beta_{2}, \sigma_{2}, \tau, Z, \mathbf{t}, \mathbf{x}\right) \propto$

$$
\exp \left\{-\frac{1}{2 s_{1}^{2}}\left(\gamma-\gamma_{0}\right)^{2}\right\} \Psi_{1}(\Theta)
$$


onde $\Psi_{1}(\Theta)=\exp \left\{\gamma r-\sum_{k=1}^{K} \sum_{i=1}^{n} \ln \left(1+e^{\gamma+\tau x_{i k}}\right)\right\}$

(viii)

$$
\pi\left(\tau \mid \alpha_{1}, \beta_{1}, \sigma_{1}, \alpha_{2}, \beta_{2}, \sigma_{2}, \gamma, Z, \mathbf{t}, \mathbf{x}\right) \propto
$$

$$
\exp \left\{-\frac{1}{2 s_{2}^{2}}\left(\tau-\tau_{0}\right)^{2}\right\} \Psi_{2}(\Theta)
$$

onde $\Psi_{2}(\Theta)=\exp \left\{\tau a_{1}-\sum_{k=1}^{K} \sum_{i=1}^{n} \ln \left(1+e^{\gamma+\tau x_{i k}}\right)\right\}$

Como podemos observar as variáveis $\gamma$ e $\tau$ devem ser geradas usando o algoritmo Metropolis-Hastings.

\subsection{Mistura das Distribuições Gamma-Normal}

Considere a mistura das distribuições gamma-normal no modelo (1.1), com densidades,

$$
\begin{aligned}
& f_{1}\left(t_{i k} \mid x_{i k}, \beta_{1}\right)=\frac{1}{\Gamma\left(\alpha_{0}\right)}\left(\alpha_{1}+\beta_{1} x_{i k}\right)^{\alpha_{0}} t_{i k}^{\alpha_{i}-1} \exp \left\{-\left(\alpha_{1}+\beta_{1} x_{i k}\right) t_{i k}\right\} \\
& f_{2}\left(t_{i k} \mid x_{i k}, \beta_{2}\right)=\frac{1}{\sqrt{2 \pi} \sigma} \exp \left\{-\frac{1}{2 \sigma^{2}}\left(t_{i k}-\left(\alpha_{2}+\beta_{2} x_{i k}\right)\right)^{2}\right\}
\end{aligned}
$$

onde: $\beta_{1}=\left(\alpha_{0}, \alpha_{1}, \beta_{1}\right) ; \beta_{2}=\left(\alpha_{2}, \beta_{2}, \sigma\right) ; \alpha_{0}>0$;

$\left(\alpha_{1}+\beta_{1} x_{i k}\right)>0 ;-\infty<t_{i k}<+\infty ;-\infty<\left(\alpha_{2}+\beta_{2} x_{i k}\right)<+\infty$; e o mesmo modelo de regressão logística (3.4) para modelar as probabilidades de incidência 
Consideramos as seguintes densidades à priori para $\alpha_{0}, \alpha_{1}, \beta_{1}, \alpha_{2}, \beta_{2}$, $\sigma, \gamma$ e $\tau$

(i) $\alpha_{0}, \alpha_{1}, \beta_{1}, \alpha_{2}, \beta_{2}, \sigma$ localmente uniformes;

(ii) $\gamma \sim N\left(\gamma_{0}, s_{1}^{2}\right), \gamma_{0}, s_{1}^{2}$ conhecidos;

(iii) $\tau \sim N\left(\tau_{0}, s_{2}^{2}\right), \tau_{0}, s_{2}^{2}$ conhecidos.

onde assumimos independência à priori entre os parâmetros.

Considerando a variável artificial $Z$, a densidade à posteriori conjuta para $\Theta=\left(\alpha_{0}, \alpha_{1}, \beta_{1}, \alpha_{2}, \beta_{2}, \sigma, \gamma, \tau\right)$, é dada por:

$$
\begin{aligned}
& \pi(\Theta \mid \mathbf{t}, Z, \mathbf{x}) \propto \frac{e^{\gamma r+r a_{1}}}{\sigma^{(n-r)}\left\{\Gamma\left(\alpha_{0}\right)\right\}^{r}\left\{\prod_{k=1}^{K} \prod_{i=1}^{n}\left(1+e^{\gamma+\tau x_{i k}}\right)\right\}} \\
& \exp \left\{-\frac{1}{2 s_{1}^{2}}\left(\gamma-\gamma_{0}\right)^{2}-\frac{1}{2 s_{2}^{2}}\left(\tau-\tau_{0}\right)^{2}\right\} \\
& \left\{\prod_{k=1}^{K} \prod_{i=1}^{n}\left(\alpha_{1}+\beta_{1} x_{i k}\right)^{\alpha_{0} z_{i k 1}}\right\}\left\{\prod_{k=1}^{K} \prod_{i=1}^{n} t_{i k}^{z_{i k 1}\left(\alpha_{0}-1\right)}\right\} \\
& \exp \left\{-\sum_{k=1}^{K} \sum_{i=1}^{n} z_{i k 1} t_{i k}\left(\alpha_{1}+\beta_{1} x_{i k}\right)^{2}\right\} \\
& \exp \left\{-\sum_{k=1}^{K} \sum_{i=1}^{n} z_{i k 2}\left(t_{i k}-\left(\alpha_{2}+\beta_{2} x_{i k}\right)\right)^{2}\right\}
\end{aligned}
$$

onde $r ; a_{1}$ e $(n-r)$ são definidos em (3.14). 
Assim, temos as seguintes distribuições condicionais para o algoritmo amostrador de Gibbs,

$$
\pi\left(\alpha_{0} \mid \alpha_{1}, \beta_{1}, \alpha_{2}, \beta_{2}, \sigma, \gamma, \tau, Z, \mathbf{t}, \mathbf{x}\right) \propto \alpha_{0}^{\sum_{k=1}^{K} \sum_{i=1}^{n} z_{i k 1}}
$$

$$
\exp \left\{-\alpha_{0} \sum_{k=1}^{K} \sum_{i=1}^{n} z_{i k 1} x_{i k}\right\} \Psi_{1}(\Theta)
$$

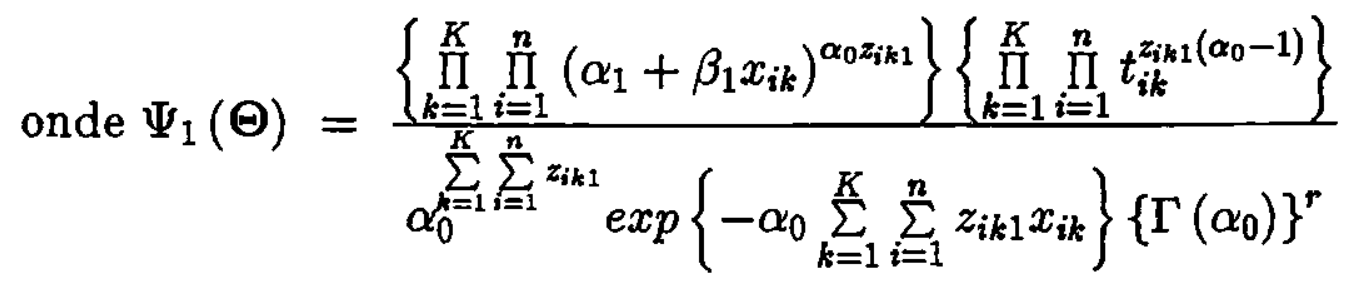

(ii)

$$
\begin{aligned}
& \pi\left(\alpha_{1} \mid \alpha_{0}, \beta_{1}, \alpha_{2}, \beta_{2}, \sigma, \gamma, \tau, Z, \mathbf{t}, \mathbf{x}\right) \propto \alpha_{1}{ }^{\alpha} \sum_{k=1}^{K} \sum_{i=1}^{n} z_{i k 1} \\
& \exp \left\{-\alpha_{1} \sum_{k=1}^{K} \sum_{i=1}^{n} z_{i k 1} t_{i k}\left(1+\frac{\beta_{1}}{\alpha_{1}} x_{i k}\right)\right\} \Psi_{2}(\Theta)
\end{aligned}
$$

onde, $\Psi_{2}(\Theta)=\prod_{k=1}^{K} \prod_{i=1}^{n}\left(1+\frac{\beta_{1}}{\alpha_{1}} x_{i k}\right)^{\alpha_{0} z_{i k 1}}$

$$
\begin{aligned}
& \pi\left(\beta_{1} \mid \alpha_{0}, \alpha_{1}, \alpha_{2}, \beta_{2}, \sigma, \gamma, \tau, Z, \mathbf{t}, \mathbf{x}\right) \propto \beta_{1}^{\alpha_{0}} \sum_{k=1}^{K} \sum_{i=1}^{n} z_{i k 1} \\
& \exp \left\{-\beta_{1} \sum_{k=1}^{K} \sum_{i=1}^{n} z_{i k 1} t_{i k}\left(\frac{\beta_{1}}{\alpha_{1}}+x_{i k}\right)\right\} \Psi_{3}(\Theta)
\end{aligned}
$$

onde, $\Psi_{3}(\Theta)=\prod_{k=1}^{K} \prod_{i=1}^{n}\left(\frac{\alpha_{1}}{\beta_{1}}+x_{i k}\right)^{\alpha_{0} z_{i k 1}}$ 
(iv)

$$
\begin{aligned}
& \pi\left(v \mid \alpha_{0}, \alpha_{1}, \beta_{1}, \alpha_{2}, \beta_{2}, \gamma, \tau, Z, \mathbf{t}, \mathbf{x}\right) \sim \\
& \Gamma\left(\frac{(n-r)}{2}+1, \frac{\sum_{k=1}^{K} \sum_{i=1}^{n} z_{i k 2}\left(t_{i k}-\left(\alpha_{2}+\beta_{2} x_{i k}\right)\right)^{2}}{2}\right)
\end{aligned}
$$

onde $v=\sigma^{-2}$

(v) $\quad \pi\left(\alpha_{2} \mid \alpha_{0}, \alpha_{1}, \beta_{1}, \beta_{2}, \sigma, \gamma, \tau, Z, \mathbf{t}, \mathbf{x}\right) \sim$

$$
N\left(\frac{\sum_{k=1}^{K} \sum_{i=1}^{n} z_{i k 2}\left(t_{i k}-\beta_{2} x_{i k}\right)}{(n-r)}, \frac{\sigma^{2}}{(n-r)}\right)
$$

(vi) $\quad \pi\left(\beta_{2} \mid \alpha_{0}, \alpha_{1}, \beta_{1}, \alpha_{2}, \sigma, \gamma, \tau, Z, \mathbf{t}, \mathbf{x}\right) \sim$

$$
\begin{gathered}
N\left(\frac{\sum_{k=1}^{K} \sum_{i=1}^{n} z_{i k 2} x_{i k}\left(t_{i k}-\alpha_{2}\right)}{\sum_{k=1}^{K} \sum_{i=1}^{n} z_{i k 2} x_{i k}^{2}}, \frac{\sigma^{2}}{\sum_{k=1}^{K} \sum_{i=1}^{n} z_{i k 2} x_{i k}^{2}}\right) \\
\left.\gamma \mid \alpha_{0}, \alpha_{1}, \beta_{1}, \sigma_{1}, \alpha_{2}, \beta_{2}, \sigma, \tau, Z, \mathbf{t}, \mathbf{x}\right) \propto \\
<p\left\{-\frac{1}{2 s_{1}^{2}}\left(\gamma-\gamma_{0}\right)^{2}\right\} \Psi_{4}(\Theta),
\end{gathered}
$$$$
e, \Psi_{4}(\Theta)=\left\{\gamma r-\sum_{k=1}^{K} \sum_{i=1}^{n} \ln \left(1+e^{\gamma+\tau x_{i k}}\right)\right\}
$$

(viii) $\quad \pi\left(\tau \mid \alpha_{0}, \alpha_{1}, \beta_{1}, \sigma_{1}, \alpha_{2}, \beta_{2}, \sigma_{2}, \gamma, Z, \mathbf{t}, \mathbf{x}\right) \propto$

$$
\exp \left\{-\frac{1}{2 s_{2}^{2}}\left(\tau-\tau_{0}\right)^{2}\right\} \Psi_{5}(\Theta)
$$


onde, $\Psi_{5}(\Theta)=\exp \left\{\tau a_{1}-\sum_{k=1}^{K} \sum_{i=1}^{n} \ln \left(1+e^{\gamma+\tau x_{i k}}\right)\right\}$.

As variáveis $\alpha_{0}, \alpha_{1}, \beta_{1}, \gamma$ e $\tau$ são geradas usando o algoritmo Metropolis-Hastings. 


\section{Capítulo 4}

\section{Modelos de Mistura para Dados com Censura Tipo II}

Apresentamos neste capítulo uma análise Bayesiana dos modelos de mistura (1.1), considerando tempos de sobrevivência censurados à direita de $t_{\text {ih }}$ (censura tipo II, ver figura 2.2), na presença de apenas uma covariável $x_{i k}, i=1,2, \ldots, n, k=1,2, \ldots, K$. A função de verossimilhança dos dados, com esse tipo de censura, envolve o cálculo da mistura de integrais e da mistura de distribuições, portanto, para tentar facilitar a implementação dos algoritmos Gibbs com Metropolis-Hastings e então obter os estimadores de Monte Carlo para as quantidades à posteriori de interesse, introduzimos no modelo as variáveis artificiais $W$ e $Z$ definidas no capítulo 2, assumindo diferentes escolhas para as densidades condicionais no modelo de mistura (1.1). 


\subsection{Mistura de Duas Distribuições Potência Expo- nencial}

Consideramos no modelo (1.1), uma mistura de $J=2$ distribuições potência exponencial com densidade (1.4), onde $\theta_{j}=\left(\alpha_{j}+\beta_{j} x_{i k}\right), j=$ $1,2, k=1,2, \ldots, K$; o modelo de regressão logística dado em (3.4) para explicar as probabilidades de incidência da $j$-ésima população; as mesmas densidades à priori para $\alpha_{1}, \beta_{1}, \sigma_{1}, \delta_{1}, \alpha_{2}, \beta_{2}, \sigma_{2}, \delta_{2}, \gamma$ e $\tau$ dadas em (3.5), assumindo indepedência à priori entre os parâmetros. Portanto, a densidade à priori conjunta para $\Theta$ é definida em (3.6).

Neste caso a função de verossimilhança (F.V.) pode ser expressa como em (2.2), e dada por:

$$
\begin{gathered}
L(\Theta \mid \mathrm{t}, \mathbf{x})=\prod_{k=1}^{K} \prod_{i=1}^{n_{i}}\left\{( \frac { e ^ { \gamma + \tau x _ { i k } } } { 1 + e ^ { \gamma + \tau x _ { i k } } } ) \left[w\left(\delta_{1}\right) \sigma_{1}^{-1}\right.\right. \\
\left.\quad \exp \left(-c\left(\delta_{1}\right)\left|\frac{t_{i k}-\left(\alpha_{1}+\beta_{1} x_{i k}\right)}{\sigma_{1}}\right|^{2 /\left(1+\delta_{1}\right)}\right)\right]+\left(\frac{1}{\left(1+e^{\gamma+\tau x_{i k}}\right)}\right) \\
\\
\left.\left[w\left(\delta_{2}\right) \sigma_{2}^{-1} \exp \left(-c\left(\delta_{2}\right)\left|\frac{t_{i k}-\left(\alpha_{2}+\beta_{2} x_{i k}\right)}{\sigma_{2}}\right|^{2 /\left(1+\delta_{2}\right)}\right)\right]\right\} \bullet
\end{gathered}
$$




$$
\begin{aligned}
& \prod_{k=1}^{K} \prod_{i=n_{i}+1}^{n}\left\{1-\left[\left\{\left(\frac{e^{\gamma+\tau x_{i k}}}{1+e^{\gamma+\tau x_{i k}}}\right)\left(\frac{\left(1+\delta_{1}\right) w\left(\delta_{1}\right) \Gamma\left(\frac{\delta_{1}+1}{2}\right)}{\left[c\left(\delta_{1}\right)\right]^{\left(\delta_{1}+1\right) / 2}}\right)\right\}\right.\right. \\
& I_{\frac{\left(\delta_{1}+1\right)}{2}}\left\{c\left(\delta_{1}\right)\left|\frac{t_{i h k}-\left(\alpha_{1}+\beta_{1} x_{i k}\right)}{\sigma_{1}}\right|^{2 /\left(1+\delta_{1}\right)}\right\}+ \\
& \left\{\left(\frac{1}{1+e^{\gamma+\tau x_{i k}}}\right)\left(\frac{\left(1+\delta_{2}\right) w\left(\delta_{2}\right) \Gamma\left(\frac{\delta_{2}+1}{2}\right)}{\left[c\left(\delta_{2}\right)\right]^{\left(\delta_{2}+1\right) / 2}}\right)\right\} \\
& \left.\left.I_{\frac{\left(\delta_{2}+1\right)}{2}}\left\{c\left(\delta_{2}\right)\left|\frac{t_{i h k}-\left(\alpha_{2}+\beta_{2} x_{i k}\right)}{\sigma_{2}}\right|^{2 /\left(1+\delta_{2}\right)}\right\}\right]\right\}
\end{aligned}
$$

onde:

$$
I_{k}(s)=\frac{1}{\Gamma(k)} \int_{0}^{s} x^{k-1} e^{-x} d x
$$

ver Demonstração (Apêndice A)

Como podemos observar em (4.1), temos o produto da mistura de integrais e da mistura de distribuições, o que torna bastante complicado a implementação do algoritmo amostrador de Gibbs se considerarmos uma F.V. dessa forma. Portanto, para tentar simplificar a implementação desse algoritmo, primeiramente eliminaremos da F.V. o cálculo do produto de integrais, introduzindo no modelo (1.1) a variável artificial $W$ a qual leva uma F.V. de observações incompletas para uma F.V. para observações iid, e que pode ser gerada pela densidade truncada (2.4) definida no capítulo 2. Assim, a F.V. (4.1) pode ser expressa como em (2.1) com $w_{i}$ no lugar de $t_{i}$, ou seja, 


$$
\begin{aligned}
L(\Theta \mid W, \mathbf{x})= & \prod_{k=1}^{K} \prod_{i=1}^{n}\left\{( \frac { e ^ { \gamma + \tau x _ { i k } } } { 1 + e ^ { \gamma + \tau x _ { i k } } } ) \left[w\left(\delta_{1}\right) \sigma_{1}^{-1}\right.\right. \\
& \left.\exp \left(-c\left(\delta_{1}\right)\left|\frac{w_{i k}-\left(\alpha_{1}+\beta_{1} x_{i k}\right)}{\sigma_{1}}\right|^{2 /\left(1+\delta_{1}\right)}\right)\right]+\left(\frac{1}{\left(1+e^{\gamma+\tau x_{i k}}\right)}\right) \\
& {\left.\left[w\left(\delta_{2}\right) \sigma_{2}^{-1} \exp \left(-c\left(\delta_{2}\right)\left|\frac{w_{i k}-\left(\alpha_{2}+\beta_{2} x_{i k}\right)}{\sigma_{2}}\right|^{2 /\left(1+\delta_{2}\right)}\right)\right]\right\} }
\end{aligned}
$$

Portanto a densidade à posteriori conjunta para $\Theta$, é dada por:

$$
\begin{aligned}
& \pi(\Theta \mid W, \mathbf{x}) \propto \pi(\Theta) \prod_{k=1}^{K} \prod_{i=1}^{n}\left\{( \frac { e ^ { \gamma + \tau x _ { i k } } } { 1 + e ^ { \gamma + \tau x _ { i k } } } ) \left[w\left(\delta_{1}\right) \sigma_{1}^{-1}\right.\right. \\
&\left.\exp \left(-c\left(\delta_{1}\right)\left|\frac{w_{i k}-\left(\alpha_{1}+\beta_{1} x_{i k}\right)}{\sigma_{1}}\right|^{2 /\left(1+\delta_{1}\right)}\right)\right]+\left(\frac{1}{\left(1+e^{\gamma+\tau x_{i k}}\right)}\right) \\
& {\left.\left[w\left(\delta_{2}\right) \sigma_{2}^{-1} \exp \left(-c\left(\delta_{2}\right)\left|\frac{w_{i k}-\left(\alpha_{2}+\beta_{2} x_{i k}\right)}{\sigma_{2}}\right|^{2 /\left(1+\delta_{2}\right)}\right)\right]\right\} }
\end{aligned}
$$

Continuamos ainda com o produto da mistura de distribuições e para simplificar mais o algoritmo amostrador de Gibbs, devemos transformar o modelo de mistura (1.1) em um modelo de componentes independentes, introduzindo a variável artificial $Z$ com densidade (2.10) definida no capítulo 2. Assim, a densidade conjunta à posteriori de $\Theta$, combinando a densidade (2.10) com a F.V. (4.3), é dada como em (3.9), ou seja, 


$$
\left.\pi(\Theta \mid W, Z, \mathbf{x}) \propto \pi(\Theta)\left\{\prod_{k=1}^{K} \prod_{i=1}^{n} \prod_{j=1}^{2}\left\{P\left(j \mid x_{i k}, \gamma_{\mathbf{j}}\right) f_{j}\left(w_{i k} \mid x_{i k}, \beta_{\mathbf{j}}\right)\right)\right\}^{z_{i k j}}\right\}
$$

que neste caso pode ser escrita como,

$$
\begin{aligned}
& \pi(\Theta \mid W, Z, \mathbf{x}) \propto \pi(\Theta)\left\{\frac{\exp \left(r \gamma+a_{1} \tau\right)}{\prod_{k=1}^{K} \prod_{i=1}^{n}\left(1+e^{\gamma+\tau x_{i k}}\right)}\right\} \frac{\left[w ( \delta _ { 1 } ] ^ { r } \left[w\left(\delta_{2}\right]^{(n-r)}\right.\right.}{\sigma_{1}^{r} \sigma_{2}^{(n-r)}} \\
& \exp \left\{-\frac{c\left(\delta_{1}\right)}{\sigma_{1}^{2 /\left(1+\delta_{1}\right)}} B_{1}\left(\alpha_{1}, \beta_{1}, \delta_{1}\right)-\frac{c\left(\delta_{2}\right)}{\sigma_{2}^{2 /\left(1+\delta_{2}\right)}} B_{2}\left(\alpha_{2}, \beta_{2}, \delta_{2}\right)\right\}
\end{aligned}
$$

onde:

$\pi(\Theta)$ é dado em (3.6); $r=\sum_{k=1}^{K} \sum_{i=1}^{n} z_{i k 1}$

$(n-r)=\sum_{k=1}^{K} \sum_{i=1}^{n} z_{i k 2}, a_{1}=\sum_{k=1}^{K} \sum_{i=1}^{n} x_{i k} z_{i k 1}$

$B_{1}\left(\alpha_{1}, \beta_{1}, \delta_{1}\right)=\sum_{k=1}^{K} \sum_{i=1}^{n} z_{i k 1}\left|w_{i k}-\left(\alpha_{1}+\beta_{1} x_{i k}\right)\right|^{2 /\left(1+\delta_{1}\right)}$

$B_{2}\left(\alpha_{2}, \beta_{2}, \delta_{2}\right)=\sum_{k=1}^{K} \sum_{i=1}^{n} z_{i k 2}\left|w_{i k}-\left(\alpha_{2}+\beta_{2} x_{i k}\right)\right|^{2 /\left(1+\delta_{2}\right)}$

Para gerarmos amostras da densidade conjunta (4.6), seguimos os passos (i), (ii) e (iii) do algoritmo de Gibbs (3.3), onde as distribuições condicionais marginais para $\alpha_{1}, \beta_{1}, \sigma_{1}, \delta_{1}, \alpha_{2}, \beta_{2}, \sigma_{2}, \delta_{2}, \gamma$, e $\tau$, são as mesmas definidas em (3.11) com $w_{i}$ no lugar de $t_{i}$, 
(i) $\pi\left(\alpha_{1} \mid \beta_{1}, \sigma_{1}, \delta_{1}, \alpha_{2}, \beta_{2}, \sigma_{2}, \delta_{2}, \gamma, \tau, W, Z, \mathbf{x}\right) \propto$

$$
\exp \left\{-\frac{1}{2 \sigma_{11}^{2}}\left(\alpha_{1}-\alpha_{10}\right)^{2}\right\} \Psi_{1}(\Theta)
$$

onde,

$$
\Psi_{1}(\Theta)=\exp \left\{-\frac{c\left(\delta_{1}\right)}{\sigma_{1}^{2 /\left(1+\delta_{1}\right)}} B_{1}\left(\alpha_{1}, \beta_{1}, \delta_{1}\right)\right\}
$$

(ii)

$$
\begin{aligned}
& \pi\left(\beta_{1} \mid \alpha_{1}, \sigma_{1}, \delta_{1}, \alpha_{2}, \beta_{2}, \sigma_{2}, \delta_{2}, \gamma, \tau, W, Z, \mathbf{x}\right) \propto \\
& \exp \left\{-\frac{1}{2 \sigma_{12}^{2}}\left(\beta_{1}-\beta_{10}\right)^{2}\right\} \Psi_{2}(\Theta)
\end{aligned}
$$

onde,

$$
\Psi_{2}(\Theta)=\exp \left\{-\frac{c\left(\delta_{1}\right)}{\sigma_{1}^{2 /\left(1+\delta_{1}\right)}} B_{1}\left(\alpha_{1}, \beta_{1}, \delta_{1}\right)\right\}
$$

$$
\begin{aligned}
& \pi\left(\sigma_{1} \mid \alpha_{1}, \beta_{1}, \delta_{1}, \alpha_{2}, \beta_{2}, \sigma_{2}, \delta_{2}, \gamma, \tau, W, Z, \mathbf{x}\right) \propto \\
& \sigma_{1}^{m_{11}-1} \exp \left\{-n_{11} \sigma_{1}\right\} \Psi_{3}(\Theta)
\end{aligned}
$$

onde,

$$
\Psi_{3}(\Theta)=\exp \left\{-r \ln \left(\sigma_{1}\right)-\sigma_{1}^{-2 /\left(1+\delta_{1}\right)} c\left(\delta_{1}\right) B_{1}\left(\alpha_{1}, \beta_{1}, \delta_{1}\right)\right\}
$$

$$
\begin{aligned}
& \pi\left(\delta_{1} \mid \alpha_{1}, \beta_{1}, \sigma_{1}, \alpha_{2}, \beta_{2}, \sigma_{2}, \delta_{2}, \gamma, \tau, W, Z, \mathbf{x}\right) \propto \\
& \exp \left\{-\frac{1}{2 \sigma_{13}^{2}}\left(\delta_{1}-\delta_{10}\right)^{2}\right\} \Psi_{4}(\Theta)
\end{aligned}
$$

onde, 


$$
\Psi_{4}(\Theta)=\exp \left\{r \ln \left(w\left(\delta_{1}\right)\right)-\frac{c\left(\delta_{1}\right)}{\sigma_{1}^{2 /\left(1+\delta_{1}\right)}} B_{1}\left(\alpha_{1}, \beta_{1}, \delta_{1}\right)\right\}
$$

(v) $\quad \pi\left(\alpha_{2} \mid \alpha_{1}, \beta_{1}, \sigma_{1}, \delta_{1}, \beta_{2}, \sigma_{2}, \delta_{2}, \gamma, \tau, W, Z, \mathbf{x}\right) \propto$

$$
\exp \left\{-\frac{1}{2 \sigma_{21}^{2}}\left(\alpha_{2}-\alpha_{20}\right)^{2}\right\} \Psi_{5}(\Theta)
$$

onde,

$$
\Psi_{5}(\Theta)=\exp \left\{-\frac{c\left(\delta_{2}\right)}{\sigma_{2}^{2 /\left(1+\delta_{2}\right)}} B_{2}\left(\alpha_{2}, \beta_{2}, \delta_{2}\right)\right\}
$$

(vi)

$$
\begin{aligned}
& \pi\left(\beta_{2} \mid \alpha_{1}, \beta_{1}, \sigma_{1}, \delta_{1}, \alpha_{2}, \sigma_{2}, \delta_{2}, \gamma, \tau, W, Z, \mathbf{x}\right) \propto \\
& \exp \left\{-\frac{1}{2 \sigma_{22}^{2}}\left(\beta_{2}-\beta_{20}\right)^{2}\right\} \Psi_{6}(\Theta)
\end{aligned}
$$

onde,

$$
\Psi_{6}(\Theta)=\exp \left\{-\frac{c\left(\delta_{2}\right)}{\dot{\sigma}_{2}^{2 /\left(1+\delta_{2}\right)}} B_{2}\left(\alpha_{2}, \beta_{2}, \delta_{2}\right)\right\}
$$

(vii) $\quad \pi\left(\sigma_{2} \mid \alpha_{1}, \beta_{1}, \delta_{1}, \sigma_{1}, \alpha_{2}, \beta_{2}, \delta_{2}, \gamma, \tau, W, Z, \mathbf{x}\right) \propto$

$$
\sigma_{2}^{m_{22}-1} \exp \left\{-n_{22} \sigma_{2}\right\} \Psi_{7}(\Theta)
$$

onde,

$$
\Psi_{7}(\Theta)=\exp \left\{-(n-r) \ln \left(\sigma_{2}\right)-\sigma_{2}^{-2 /\left(1+\delta_{2}\right)} c\left(\delta_{2}\right) B_{2}\left(\alpha_{2}, \beta_{2}, \delta_{2}\right)\right\}
$$


(viii) $\quad \pi\left(\delta_{2} \mid \alpha_{1}, \beta_{1}, \sigma_{1}, \delta_{1}, \alpha_{2}, \beta_{2}, \sigma_{2}, \gamma, \tau, W, Z, \mathbf{x}\right) . \propto$

$$
\exp \left\{-\frac{1}{2 \sigma_{23}^{2}}\left(\delta_{2}-\delta_{20}\right)^{2}\right\} \Psi_{8}(\Theta)
$$

onde,

$$
\Psi_{8}(\Theta)=\exp \left\{(n-r) \ln \left(w\left(\delta_{2}\right)\right)-\frac{c\left(\delta_{2}\right)}{\sigma_{2}^{2 /\left(1+\delta_{2}\right)}} B_{2}\left(\alpha_{2}, \beta_{2}, \delta_{2}\right)\right\}
$$

(ix) $\quad \pi\left(\gamma \mid \alpha_{1}, \beta_{1}, \sigma_{1}, \delta_{1}, \alpha_{2}, \beta_{2}, \sigma_{2}, \delta_{2}, \tau, W, Z, \mathbf{x}\right) \propto$

$$
\exp \left\{-\frac{1}{2 \sigma_{14}^{2}}\left(\gamma-\gamma_{0}\right)^{2}\right\} \Psi_{9}(\Theta)
$$

onde,

$$
\Psi_{9}(\Theta)=\exp \left\{r \gamma-\sum_{k=1}^{K} \sum_{i=1}^{n} \ln \left(1+e^{\gamma+\tau x_{i k}}\right)\right\}
$$

$$
\begin{aligned}
& \pi\left(\tau \mid \alpha_{1}, \beta_{1}, \sigma_{1}, \delta_{1}, \alpha_{2}, \beta_{2}, \sigma_{2}, \delta_{2}, \gamma, W, Z, \mathbf{x}\right) \propto \\
& \exp \left\{-\frac{1}{2 \sigma_{24}^{2}}\left(\tau-\tau_{0}\right)^{2}\right\} \Psi_{10}(\Theta)
\end{aligned}
$$

onde,

$$
\Psi_{10}(\Theta)=\exp \left\{a_{1} \tau-\sum_{k=1}^{K} \sum_{i=1}^{n} \ln \left(1+e^{\gamma+\tau x_{i k}}\right)\right\}
$$

Observe que as densidades (4.7) não tem uma forma padrão conhecida, portanto devemos usar o algoritmo Metropolis-Hastings para gerar todos os parâmetros de $\Theta$. 


\subsection{Mistura das Distribuições Normal-Exponencial}

Consideramos no modelo de mistura (1.1) as probabilidades de incidência (3.4) e as distribuições de latência condicionais para os tempos de sobrevivência $t$, com densidades (3.12).

Assumindo independência à priori entre os parâmetros, consideramos as mesmas densidades à priori para $\alpha_{1}, \beta_{1}, \sigma, \alpha_{2}, \beta_{2}, \gamma$ e $\tau$ dadas em (3.13), e a F.V. definida em (2.2).

Com o objetivo de tentar simplificar o algoritmo amostrador de Gibbs, introduzimos as variáveis artificiais $W$ e $Z$ respectivamente, assim, a densidade à posteriori conjunta de $\Theta=\left(\alpha_{1}, \beta_{1}, \sigma, \alpha_{2}, \beta_{2}, \gamma, \tau\right)$ pode ser expressa como em (3.14) mas com $w_{i}$ no lugar de $t_{i}$, ou seja,

$$
\begin{aligned}
& \pi(\Theta \mid W, Z, \mathbf{x}) \propto \frac{\left\{\prod_{k=1}^{K} \prod_{i=1}^{n}\left(\alpha_{2}+\beta_{2} x_{i k}\right)^{-z_{i k 2}}\right\}}{\left\{\prod_{k=1}^{K} \prod_{i=1}^{n}\left(1+e^{\gamma+r x_{i k}}\right)\right\}} \frac{e^{\gamma r+\tau a_{1}}}{\sigma^{\sigma}} \\
& \exp \left\{-\frac{1}{2 s_{1}^{2}}\left(\gamma-\gamma_{0}\right)^{2}-\frac{1}{2 s_{2}^{2}}\left(\tau-\tau_{0}\right)^{2}\right\} \\
& \left\{\operatorname { e x p } \left\{-\frac{1}{2 \sigma^{2}} \sum_{k=1}^{K} \sum_{i=1}^{n} z_{i k 1}\left(w_{i k}-\left(\alpha_{1}+\beta_{1} x_{i k}\right)\right)^{2}-\right.\right. \\
& \left.\left.\sum_{k=1}^{K} \sum_{i=1}^{n} z_{i k 2} w_{i k}\left(\alpha_{2}+\beta_{2} x_{i k}\right)^{-1}\right\}\right\}
\end{aligned}
$$

onde $r=\sum_{k=1}^{K} \sum_{i=1}^{n} z_{i k 1} ;(n-r)=\sum_{k=1}^{K} \sum_{i=1}^{n} z_{i k 2} \mathrm{e}$ 
$a_{1}=\sum_{k=1}^{K} \sum_{i=1}^{n} x_{i k} z_{i k 1}$

Portanto, geramos amostras da densidade à posteriori conjunta (4.8), seguindo os passos (i), (ii) e (iii) do algoritmo de Gibbs (3.3), onde as distribuições condicionais para os parâmetros são as mesmas dadas em (3.15), considerando $w_{i}$ no lugar de $t_{i}$, isto é,

(i)

$$
\begin{aligned}
& \pi\left(v \mid \alpha_{1}, \beta_{1}, \alpha_{2}, \beta_{2}, \gamma, \tau, W, Z, \mathbf{x}\right) \sim \\
& \Gamma\left(\frac{r}{2}+1, \frac{\sum_{k=1}^{K} \sum_{i=1}^{n} z_{i k 1}\left(w_{i k}-\left(\alpha_{1}+\beta_{1} x_{i k}\right)\right)^{2}}{2}\right)
\end{aligned}
$$

onde $v=\sigma^{-2}$

(ii)

$$
\begin{aligned}
& \pi\left(\alpha_{1} \mid \beta_{1}, \sigma, \alpha_{2}, \beta_{2}, \gamma, \tau, W, Z, \mathbf{x}\right) \sim \\
& N\left(\frac{\sum_{k=1}^{K} \sum_{i=1}^{n} z_{i k 1}\left(w_{i k}-\beta_{1} x_{i k}\right)}{r}, \frac{\sigma^{2}}{r}\right)
\end{aligned}
$$

(iii)

$$
\pi\left(\beta_{1} \mid \alpha_{1}, \sigma, \alpha_{2}, \beta_{2}, \gamma, \tau, W, Z, \mathbf{x}\right) \sim
$$

$$
N\left(\frac{\sum_{k=1}^{K} \sum_{i=1}^{n} z_{i k 1} x_{i k}\left(w_{i k}-\alpha_{1}\right)}{\sum_{k=1}^{K} \sum_{i=1}^{n} z_{i k 1} x_{i k}^{2}}, \frac{\sigma^{2}}{\sum_{k=1}^{K} \sum_{i=1}^{n} z_{i k 1} x_{i k}^{2}}\right)
$$

(iv) $\quad \pi\left(\alpha_{2} \mid \alpha_{1}, \beta_{1}, \sigma, \beta_{2}, \gamma, \tau, W, Z, \mathbf{x}\right) \propto \alpha_{2}^{-(n-r)}$

$$
\exp \left\{-\frac{1}{\alpha_{2}} \sum_{k=1}^{K} \sum_{i=1}^{n} z_{i k 2} w_{i k}\left(1+\frac{\beta_{2}}{\alpha_{2}} x_{i k}\right)^{-1}\right\} \Psi_{1}(\Theta)
$$


onde $\Psi_{1}(\Theta)=\prod_{k=1}^{K} \prod_{i=1}^{n}\left(1+\frac{\beta_{2}}{\alpha_{2}} x_{i k}\right)^{-z_{i k 2}}$

(v) $\quad \pi\left(\beta_{2} \mid \alpha_{1}, \beta_{1}, \sigma, \alpha_{2}, \gamma, \tau, W, Z, \mathbf{x}\right) \propto \beta_{2}^{-(n-r)}$

$$
\exp \left\{-\frac{1}{\beta_{2}} \sum_{k=1}^{K} \sum_{i=1}^{n} z_{i k 2} w_{i k}\left(\frac{\alpha_{2}}{\beta_{2}}+x_{i k}\right)^{-1}\right\} \Psi_{2}(\Theta)
$$

onde $\Psi_{2}(\Theta)=\prod_{k=1}^{K} \prod_{i=1}^{n}\left(\frac{\alpha_{2}}{\beta_{2}}+x_{i k}\right)^{-z_{i k 2}}$

(vi) $\quad \pi\left(\gamma \mid \alpha_{1}, \beta_{1}, \sigma, \alpha_{2}, \beta_{2}, \tau, W, Z, \mathbf{x}\right) \propto$

$$
\exp \left\{-\frac{1}{2 s_{1}^{2}}\left(\gamma-\gamma_{0}\right)^{2}\right\} \Psi_{3}(\Theta)
$$

onde $\Psi_{3}(\Theta)=\exp \left\{\gamma r-\sum_{k=1}^{K} \sum_{i=1}^{n} \ln \left(1+e^{\gamma+\tau x_{i k}}\right)\right\}$

$$
\begin{aligned}
& \pi\left(\tau \mid \alpha_{1}, \beta_{1}, \sigma, \alpha_{2}, \beta_{2}, \gamma, W, Z, \mathbf{x}\right) \propto \\
& \left.\exp \left\{-\frac{1}{2 s_{2}^{2}}\left(\tau-\tau_{0}\right)^{2}\right)\right\} \Psi_{4}(\Theta)
\end{aligned}
$$

onde $\Psi_{4}(\Theta)=\exp \left\{r a_{1}-\sum_{k=1}^{K} \sum_{i=1}^{n} \ln \left(1+e^{\gamma+\tau x_{i k}}\right)\right\}$

Aqui, $\Gamma(a, b)$ denota uma distribuição gamma com média $a / b$ e variância $a / b^{2}$.

Como podemos observar, precisamos usar o algoritmo MetropolisHastings para gerar as variáveis $\alpha_{2}, \beta_{2}, \gamma$ e $\tau$. Onde geramos candidatos para $\alpha_{2}$ e $\beta_{2}$ de uma distribuição gamma inversa; e para as variáveis $\gamma$ e $\tau$ das distribuições normais $N\left(\gamma_{0}, s_{1}^{2}\right)$ e $N\left(\tau_{0}, s_{2}^{2}\right)$, respectivamente. 


\subsection{Mistura de duas Distribuições Normais}

Consideramos agora no modelo de mistura (1.1) duas distribuições normais com densidades (3.16); as probabilidades (3.4) para modelar as probabilidades de incidência $P\left(j \mid \mathbf{x}, \gamma_{\mathbf{j}}\right)$; as mesmas densidades à priori para $\alpha_{1}, \beta_{1}, \sigma_{1}, \alpha_{2}, \beta_{2}, \sigma_{2}, \gamma$ e $\tau$, definidas em (3.17) ,assumindo independência à priori entre os parâmetros; e a F.V. definida em (2.2).

A densidade à posteriori conjunta para $\Theta=\left(\alpha_{1}, \beta_{1}, \sigma_{1}, \alpha_{2}, \beta_{2}, \sigma_{2}, \gamma, \tau\right)$, considerando a introdução das variáveis artificiais $W$ e $Z$, pode ser expressa como em (3.18), com $w_{i}$ no lugar de $t_{i}$, ou seja,

$$
\begin{aligned}
& \pi(\Theta \mid W, Z, \mathbf{x}) \propto \frac{\sigma_{1}^{-r} \sigma_{2}^{-(n-r)}}{\left\{\prod_{k=1}^{K} \prod_{i=1}^{n}\left(1+e^{\gamma+\tau x_{i k}}\right)\right\}} \\
& \exp \left\{-\frac{1}{2 s_{1}^{2}}\left(\gamma-\gamma_{0}\right)^{2}+\gamma r-\frac{1}{2 s_{2}^{2}}\left(\tau-\tau_{0}\right)^{2}+\tau a_{1}\right\} \\
& \left\{\operatorname { e x p } \left\{-\frac{1}{2 \sigma_{1}^{2}} \sum_{k=1}^{K} \sum_{i=1}^{n} z_{i k 1}\left(w_{i k}-\left(\alpha_{1}+\beta_{1} x_{i k}\right)\right)^{2}-\right.\right. \\
& \left.\left.\frac{1}{2 \sigma_{2}^{2}} \sum_{k=1}^{K} \sum_{i=1}^{n} z_{i k 2}\left(w_{i k}-\left(\alpha_{2}+\beta_{2} x_{i k}\right)\right)^{2}\right\}\right\}
\end{aligned}
$$

onde $r,(n-r)$ e $a_{1}$ são definidos em (3.14).

Portanto as distribuições condicionais para o algoritmo Gibbs são as mesmas dadas em (3.19), considerando $w_{i}$ no lugar de $t_{i}$, 
(i)

$$
\begin{aligned}
& \pi\left(v \mid \alpha_{1}, \beta_{1}, \alpha_{2}, \beta_{2}, \dot{\sigma}_{2}, \gamma, \tau, W, Z, \mathbf{x}\right) \sim \\
& \Gamma\left(\frac{r}{2}+1, \frac{\sum_{k=1}^{K} \sum_{i=1}^{n} z_{i k 1}\left(w_{i k}-\left(\alpha_{1}+\beta_{1} x_{i k}\right)\right)^{2}}{2}\right)
\end{aligned}
$$

onde $v=\sigma_{1}^{-2}$

(ii)

$$
\begin{aligned}
& \pi\left(\alpha_{1}, \mid \beta_{1}, \sigma_{1}, \alpha_{2}, \beta_{2}, \sigma_{2}, \gamma, \tau, W, Z, \mathbf{x}\right) \sim \\
& N\left(\frac{\sum_{k=1}^{K} \sum_{i=1}^{n} z_{i k 1}\left(w_{i k}-\beta_{1} x_{i k}\right)}{r}, \frac{\sigma_{1}^{2}}{r}\right)
\end{aligned}
$$

(iii)

$$
\begin{aligned}
& \pi\left(\beta_{1} \mid \alpha_{1}, \sigma_{1}, \alpha_{2}, \beta_{2}, \sigma_{2}, \gamma, \tau, W, Z, \mathbf{x}\right) \sim \\
& N\left(\frac{\sum_{k=1}^{K} \sum_{i=1}^{n} z_{i k 1} x_{i k}\left(w_{i k}-\alpha_{1}\right)}{\sum_{k=1}^{K} \sum_{i=1}^{n} z_{i k 1} x_{i k}^{2}}, \frac{\sigma_{1}^{2}}{\sum_{k=1}^{K} \sum_{i=1}^{n} z_{i k 1} x_{i k}^{2}}\right)
\end{aligned}
$$

(iv)

$$
\begin{aligned}
& \pi\left(u \mid \alpha_{1}, \beta_{1}, \sigma_{1}, \alpha_{2}, \beta_{2}, \gamma, \tau, W, Z, \mathbf{x}\right) \sim \\
& \Gamma\left(\frac{(n-r)}{2}+1, \frac{\sum_{k=1}^{K} \sum_{i=1}^{n} z_{i k 2}\left(w_{i k}-\left(\alpha_{2}+\beta_{2} x_{i k}\right)\right)^{2}}{2}\right)
\end{aligned}
$$

onde $u=\sigma_{2}^{-2}$ 
(v) $\quad \pi\left(\alpha_{2} \mid \alpha_{1}, \beta_{1}, \sigma_{1}, \beta_{2}, \sigma_{2}, \gamma, \tau, W, Z, \mathbf{x}\right) \sim$

$$
N\left(\frac{\sum_{k=1}^{K} \sum_{i=1}^{n} z_{i k 2}\left(w_{i k}-\beta_{2} x_{i k}\right)}{(n-r)}, \frac{\sigma_{2}^{2}}{(n-r)}\right)
$$

(vi) $\quad \pi\left(\beta_{2} \mid \alpha_{1}, \beta_{1}, \sigma_{1}, \alpha_{2}, \sigma_{2}, \gamma, \tau, W, Z, \mathbf{x}\right) \sim$

$$
N\left(\frac{\sum_{k=1}^{K} \sum_{i=1}^{n} z_{i k 2} x_{i k}\left(w_{i k}-\alpha_{2}\right)}{\sum_{k=1}^{K} \sum_{i=1}^{n} z_{i k 2} x_{i k}^{2}}, \frac{\sigma_{2}^{2}}{\sum_{k=1}^{K} \sum_{i=1}^{n} z_{i k 2} x_{i k}^{2}}\right)
$$

(vii) $\quad \pi\left(\gamma \mid \alpha_{1}, \beta_{1}, \sigma_{1}, \alpha_{2}, \beta_{2}, \sigma_{2}, \tau, W, Z, \mathbf{x}\right) \propto$

$$
\exp \left\{-\frac{1}{2 s_{1}^{2}}\left(\gamma-\gamma_{0}\right)^{2}\right\} \Psi_{1}(\Theta)
$$

onde $\Psi_{1}(\Theta)=\exp \left\{\gamma r-\sum_{k=1}^{K} \sum_{i=1}^{n} \ln \left(1+e^{\gamma+\tau x_{i k}}\right)\right\}$

(viii)

$$
\pi\left(\tau \mid \alpha_{1}, \beta_{1}, \sigma_{1}, \alpha_{2}, \beta_{2}, \sigma_{2}, \gamma, W, Z, \mathbf{x}\right) \propto
$$

$$
\exp \left\{-\frac{1}{2 s_{2}^{2}}\left(\tau-\tau_{0}\right)^{2}\right\} \Psi_{2}(\Theta)
$$

onde $\Psi_{2}(\Theta)=\exp \left\{\tau a_{1}-\sum_{k=1}^{K} \sum_{i=1}^{n} \ln \left(1+e^{\gamma+\tau x_{i k}}\right)\right\}$

Como podemos observar as variáveis $\gamma$ e $\tau$ devem ser geradas usando o algoritmo Metropolis-Hastings. 


\subsection{Mistura das Distribuições Gamma-Normal}

Consideramos agora uma mistura das distribuições gamma-normal no modelo (1.1), com densidades (3.20); as probabilidades (3.4) para modelar as probabilidades de incidência $P\left(j \mid \mathbf{x}, \gamma_{\mathbf{j}}\right)$; as mesmas densidades à priori para $\alpha_{0}, \alpha_{1}, \beta_{1}, \alpha_{2}, \beta_{2}, \sigma, \gamma$ e $\tau$, definidas em (3.21), assumindo independência à priori entre os parâmetros.

Com a introdução das variáveis artificiais $W$ e $Z$, a densidade à posteriori conjuta para $\Theta=\left(\alpha_{0}, \alpha_{1}, \beta_{1}, \alpha_{2}, \beta_{2}, \sigma, \gamma, \tau\right)$, pode ser expressa como em (3.22), mas considerando $w_{i}$ no lugar de $t_{i}$, isto é,

$$
\begin{aligned}
& \pi(\Theta \mid W, Z, \mathbf{x}) \propto \frac{e^{\gamma r+r a_{1}}}{\sigma^{(n-r)}\left\{\Gamma\left(\alpha_{0}\right)\right\}^{r}\left\{\prod_{k=1}^{K} \prod_{i=1}^{n}\left(1+e^{\gamma+r x_{i k}}\right)\right\}} \\
& \exp \left\{-\frac{1}{2 s_{1}^{2}}\left(\gamma-\gamma_{0}\right)^{2}-\frac{1}{2 s_{2}^{2}}\left(\tau-\tau_{0}\right)^{2}\right\} \\
& \left\{\prod_{k=1}^{K} \prod_{i=1}^{n}\left(\alpha_{1}+\beta_{1} x_{i k}\right)^{\alpha_{0} z_{i k 1}}\right\}\left\{\prod_{k=1}^{K} \prod_{i=1}^{n} w_{i k}^{z_{i k 1}\left(\alpha_{0}-1\right)}\right\} \\
& \exp \left\{-\sum_{k=1}^{K} \sum_{i=1}^{n} z_{i k 1} w_{i k}\left(\alpha_{1}+\beta_{1} x_{i k}\right)^{2}\right\} \\
& \exp \left\{-\sum_{k=1}^{K} \sum_{i=1}^{n} z_{i k 2}\left(w_{i k}-\left(\alpha_{2}+\beta_{2} x_{i k}\right)\right)^{2}\right\}
\end{aligned}
$$

onde $r ; a_{1}$ e $(n-r)$ são definidos em (3.14).

Assim, temos as mesmas distribuições condicionais para o algoritmo 
amostrador de Gibbs dadas em (3.23), mas com $w_{i}$ no lugar de $t_{i}$, ou seja,

$$
\pi\left(\alpha_{0} \mid \alpha_{1}, \beta_{1}, \alpha_{2}, \beta_{2}, \sigma, \gamma, \tau, W, Z, \mathbf{x}\right) \propto \alpha_{0}^{\sum_{k=1}^{K} \sum_{i=1}^{n} z_{i k 1}}
$$

$$
\exp \left\{-\alpha_{0} \sum_{k=1}^{K} \sum_{i=1}^{n} z_{i k 1} x_{i k}\right\} \Psi_{1}(\Theta)
$$

onde $\Psi_{1}(\Theta)=\frac{\left\{\prod_{k=1}^{K} \prod_{i=1}^{n}\left(\alpha_{1}+\beta_{1} x_{i k}\right)^{\alpha_{0} z_{i k 1}}\right\}\left\{\prod_{k=1}^{K} \prod_{i=1}^{n} w_{i k}^{z_{i k 1}\left(\alpha_{0}-1\right)}\right\}}{\alpha_{0}^{\sum_{k=1}^{K} \sum_{i=1}^{n} z_{i k 1}} \exp \left\{-\alpha_{0} \sum_{k=1}^{K} \sum_{i=1}^{n} z_{i k 1} x_{i k}\right\}\left\{\Gamma\left(\alpha_{0}\right)\right\}^{r}}$

(ii)

$$
\begin{aligned}
& \pi\left(\alpha_{1} \mid \alpha_{0}, \beta_{1}, \alpha_{2}, \beta_{2}, \sigma, \gamma, \tau, W, Z, \mathbf{x}\right) \propto \alpha_{1}^{\alpha_{0} \sum_{k=1}^{K} \sum_{i=1}^{n} z_{i k 1}} \\
& \exp \left\{-\alpha_{1} \sum_{k=1}^{K} \sum_{i=1}^{n} z_{i k 1} w_{i k}\left(1+\frac{\beta_{1}}{\alpha_{1}} x_{i k}\right)\right\} \Psi_{2}(\Theta)
\end{aligned}
$$

onde, $\Psi_{2}(\Theta)=\prod_{k=1}^{K} \prod_{i=1}^{n}\left(1+\frac{\beta_{1}}{\alpha_{1}} x_{i k}\right)^{\alpha_{0} z_{i k 1}}$

$$
\begin{aligned}
& \pi\left(\beta_{1} \mid \alpha_{0}, \alpha_{1}, \alpha_{2}, \beta_{2}, \sigma, \gamma, \tau, W, Z, \mathbf{x}\right) \propto \beta_{1}^{\alpha_{0} \sum_{k=1}^{K} \sum_{i=1}^{n} z_{i k 1}} \\
& \exp \left\{-\beta_{1} \sum_{k=1}^{K} \sum_{i=1}^{n} z_{i k 1} w_{i k}\left(\frac{\beta_{1}}{\alpha_{1}}+x_{i k}\right)\right\} \Psi_{3}(\Theta)
\end{aligned}
$$

onde, $\Psi_{3}(\Theta)=\prod_{k=1}^{K} \prod_{i=1}^{n}\left(\frac{\alpha_{1}}{\beta_{1}}+x_{i k}\right)^{\alpha_{0} z_{i k 1}}$ 
(iv) $\quad \pi\left(v \mid \alpha_{0}, \alpha_{1}, \beta_{1}, \alpha_{2}, \beta_{2}, \gamma, \tau, W, Z, \mathbf{x}\right) \sim$

$$
\Gamma\left(\frac{(n-r)}{2}+1, \frac{\sum_{k=1}^{K} \sum_{i=1}^{n} z_{i k 2}\left(w_{i k}-\left(\alpha_{2}+\beta_{2} x_{i k}\right)\right)^{2}}{2}\right)
$$

onde $v=\sigma^{-2}$

(v) $\pi\left(\alpha_{2} \mid \alpha_{0}, \alpha_{1}, \beta_{1}, \beta_{2}, \sigma, \gamma, \tau, W, Z, \mathbf{x}\right) \sim$

$$
N\left(\frac{\sum_{k=1}^{K} \sum_{i=1}^{n} z_{i k 2}\left(w_{i k}-\beta_{2} x_{i k}\right)}{(n-r)}, \frac{\sigma^{2}}{(n-r)}\right)
$$

(vi) $\quad \pi\left(\beta_{2} \mid \alpha_{0}, \alpha_{1}, \beta_{1}, \alpha_{2}, \sigma, \gamma, \tau, W, Z, \mathbf{x}\right) \sim$

$$
N\left(\frac{\sum_{k=1}^{K} \sum_{i=1}^{n} z_{i k 2} x_{i k}\left(w_{i k}-\alpha_{2}\right)}{\sum_{k=1}^{K} \sum_{i=1}^{n} z_{i k 2} x_{i k}^{2}}, \frac{\sigma^{2}}{\sum_{k=1}^{K} \sum_{i=1}^{n} z_{i k 2} x_{i k}^{2}}\right)
$$

(vii) $\quad \pi\left(\gamma \mid \alpha_{0}, \alpha_{1}, \beta_{1}, \sigma_{1}, \alpha_{2}, \beta_{2}, \sigma, \tau, W, Z, \mathbf{x}\right) \propto$

$$
\exp \left\{-\frac{1}{2 s_{1}^{2}}\left(\gamma-\gamma_{0}\right)^{2}\right\} \Psi_{4}(\Theta)
$$

onde, $\Psi_{4}(\Theta)=\left\{\gamma r-\sum_{k=1}^{K} \sum_{i=1}^{n} \ln \left(1+e^{\gamma+\tau x_{i k}}\right)\right\}$

(viii)

$$
\begin{aligned}
& \pi\left(\tau \mid \alpha_{0}, \alpha_{1}, \beta_{1}, \sigma_{1}, \alpha_{2}, \beta_{2}, \sigma_{2}, \gamma, W, Z, \mathbf{x}\right) \propto \\
& \exp \left\{-\frac{1}{2 s_{2}^{2}}\left(\tau-\tau_{0}\right)^{2}\right\} \Psi_{5}(\Theta)
\end{aligned}
$$


onde, $\Psi_{5}(\Theta)=\exp \left\{\tau a_{1}-\sum_{k=1}^{K} \sum_{i=1}^{n} \ln \left(1+e^{\gamma+\tau x_{i k}}\right)\right\}$

As variáveis $\alpha_{0}, \alpha_{1}, \beta_{1}, \gamma$ e $\tau$ são geradas usando o algoritmo Metropolis-Hastings. 


\section{Capítulo 5}

\section{Modelos de Mistura para Dados Agrupados}

Neste capítulo, apresentamos uma análise Bayesiana dos modelos de mistura (1.1), mas agora considerando tempos de sobrevivência censurados entre os intervalos $\left(t_{i l}, t_{i u}\right)$, e censurados à direita de $t_{i h}$, (veja figura 2.3); e o mesmo vetor único de covariáveis $x_{i k}, i=1, \ldots, n, k=1, \ldots, K$, que podem influenciar os tempos de sobrevivência $t \mathrm{e}$ as probabilidades de incidência $P\left(j \mid \mathbf{x}, \gamma_{\mathbf{j}}\right)$. Portanto, utilizamos os algoritmos de Gibbs com Metropolis-Hastings e estimadores de Monte Carlo para obter as quantidades à posteriori de interesse, assumindo diferentes escolhas para as densidades condicionais no modelo de mistura (1.1). Considerando a introdução das variáveis artificiais $W$ e $Z$ para facilitar a implementação dos algoritmos de simulação de amostras. 


\subsection{Mistura de Duas Distribuições Potência Expo- nencial}

Consideramos aqui as mesmas distribuições potência exponencial com densidades (1.4); as probabilidades de incidência (3.4); e as mesmas densidades à priori para $\alpha_{1}, \beta_{1}, \sigma_{1}, \delta_{1}, \alpha_{2}, \beta_{2}, \sigma_{2}, \delta_{2}, \gamma$, e $\tau$ definidas em (3.5), assumindo independência à priori entre os parâmetros. Podemos considerar neste caso a função de verossimilhança (F.V.) definida em (2.3), dada por:

$$
\begin{aligned}
L(\Theta \mid \mathbf{t}, \mathbf{x}) & =\prod_{k=1}^{K} \prod_{i=1}^{n_{i}}\left\{\left[\left\{\left(\frac{e^{\gamma+\tau x_{i k}}}{1+e^{\gamma+\tau x_{i k}}}\right)\left(\frac{\left(1+\delta_{1}\right) w\left(\delta_{1}\right) \Gamma\left(\frac{\delta_{1}+1}{2}\right)}{\left[c\left(\delta_{1}\right)\right]^{\left(\delta_{1}+1\right) / 2}}\right)\right\}\right.\right. \\
& I_{\frac{\left(\delta_{1}+1\right)}{2}}\left\{c\left(\delta_{1}\right)\left|\frac{t_{i u k}-\left(\alpha_{1}+\beta_{1} x_{i k}\right)}{\sigma_{1}}\right|^{2 /\left(1+\delta_{1}\right)}\right\}+ \\
& \left\{\left(\frac{1}{1+e^{\gamma+\tau x_{i k}}}\right)\left(\frac{\left(1+\delta_{2}\right) w\left(\delta_{2}\right) \Gamma\left(\frac{\delta_{2}+1}{2}\right)}{\left[c\left(\delta_{2}\right)\right]^{\left(\delta_{2}+1\right) / 2}}\right)\right\} \\
& \left.I_{\frac{\left(\delta_{2}+1\right)}{2}}\left\{c\left(\delta_{2}\right)\left|\frac{t_{i u k}-\left(\alpha_{2}+\beta_{2} x_{i k}\right)}{\sigma_{2}}\right|^{2 /\left(1+\delta_{2}\right)}\right\}\right]- \\
& {\left[\left\{\left(\frac{e^{\gamma+\tau x_{i k}}}{1+e^{\gamma+\tau x_{i k}}}\right)\left(\frac{\left(1+\delta_{1}\right) w\left(\delta_{1}\right) \Gamma\left(\frac{\delta_{1}+1}{2}\right)}{\left[c\left(\delta_{1}\right)\right]^{\left(\delta_{1}+1\right) / 2}}\right)\right\}\right.}
\end{aligned}
$$




$$
\begin{aligned}
& I_{\frac{\left(\delta_{1}+1\right)}{2}}\left\{c\left(\delta_{1}\right)\left|\frac{t_{i l k}-\left(\alpha_{1}+\beta_{1} x_{i k}\right)}{\sigma_{1}}\right|^{2 /\left(1+\delta_{1}\right)}\right\}+ \\
& \left\{\left(\frac{1}{1+e^{\gamma+\tau x_{i k}}}\right)\left(\frac{\left(1+\delta_{2}\right) w\left(\delta_{2}\right) \Gamma\left(\frac{\delta_{2}+1}{2}\right)}{\left[c\left(\delta_{2}\right)\right]^{\left(\delta_{2}+1\right) / 2}}\right)\right\} \\
& \left.\left.I_{\frac{\left(\delta_{2}+1\right)}{2}}\left\{c\left(\delta_{2}\right)\left|\frac{t_{i l k}-\left(\alpha_{2}+\beta_{2} x_{i k}\right)}{\sigma_{2}}\right|^{2 /\left(1+\delta_{2}\right)}\right\}\right]\right\} \\
& \prod_{k=1}^{K} \prod_{i=n_{i}+1}^{n}\left\{1-\left[\left\{\left(\frac{e^{\gamma+\tau x_{i k}}}{1+e^{\gamma+\tau x_{i k}}}\right)\left(\frac{\left(1+\delta_{1}\right) w\left(\delta_{1}\right) \Gamma\left(\frac{\delta_{1}+1}{2}\right)}{\left[c\left(\delta_{1}\right)\right]^{\left(\delta_{1}+1\right) / 2}}\right)\right\}\right.\right. \\
& I_{\frac{\left(\delta_{1}+1\right)}{2}}\left\{c\left(\delta_{1}\right)\left|\frac{t_{i l k}-\left(\alpha_{1}+\beta_{1} x_{i k}\right)}{\sigma_{1}}\right|^{2 /\left(1+\delta_{1}\right)}\right\}+ \\
& \left\{\left(\frac{1}{1+e^{\gamma+\tau x_{i k}}}\right)\left(\frac{\left(1+\delta_{2}\right) w\left(\delta_{2}\right) \Gamma\left(\frac{\delta_{2}+1}{2}\right)}{\left[c\left(\delta_{2}\right)\right]^{\left(\delta_{2}+1\right) / 2}}\right)\right\} \\
& \left.\left.I_{\frac{\left(\delta_{2}+1\right)}{2}}\left\{c\left(\delta_{2}\right)\left|\frac{t_{i l k}-\left(\alpha_{2}+\beta_{2} x_{i k}\right)}{\sigma_{2}}\right|^{2 /\left(1+\delta_{2}\right)}\right\}\right]\right\}
\end{aligned}
$$

onde, $I_{k}(s)$ é definido em (4.2).

A F.V. (5.1) é ainda mais complexa do que a F.V. (4.1), e portanto extremamente complicado implementar os algoritmos Gibbs com MetropolisHastings. Para solucionar este problema, introduzimos no modelo (1.1) a variável artificial $W$, da qual iremos gerar os tempos censurados entre os intervalos $\left(t_{i l}, t_{i u}\right)$ à partir da densidade truncada (2.6), e os tempos censurados à direita de $t_{i h}$ à partir da densidade truncada (2.4), ambas 
definidas no capítulo 2: Com isso, podemos expressar a F.V. (5.1) como em (4.3), e a densidade à posteriori conjunta para $\Theta$ definida em (4.4).

Observando (4.4) podemos constatar que ainda continuamos com produto da mistura de distribuições, mas isso é facilmente resolvido com a introdução da variável artificial $Z$ com densidade (2.10) definida no capítulo 2, assim, combinando a densidade (2.10) com a F.V. (4.3) podemos considerar uma F.V. como em (3.7), e portanto a densidade à posteriori conjunta (4.4) pode ser escrita como em (4.6).

Finalmente podemos gerar amostras para $\alpha_{1}, \beta_{1}, \sigma_{1}, \delta_{1}, \alpha_{2}, \beta_{2}, \sigma_{2}, \delta_{2}$, $\gamma$, e $\tau$ à partir das densidades condicionais (4.7), seguindo os passos (i), (ii) e (iii) do algoritmo de Gibbs (3.3).

\subsection{Mistura das Distribuições Normal-Exponencial}

Neste caso consideramos no modelo (1.1) as mesmas probabilidades de incidência (3.4); as distribuições condicionais com densidades (3.12); as mesmas densidades à priori para $\alpha_{1}, \beta_{1}, \sigma, \alpha_{2}, \beta_{2}, \gamma$, e $\tau$ definidas em (3.13), assumindo independência entre os parâmetros; e as variáveis artificiais $W$ e $Z$ respectivamente, procedendo de acordo como descrito na seção (5.1). Portanto, a densidade à posteriori conjunta de $\Theta$ pode ser escrita como em (4.8), e assim podemos gerar amostras à partir das densidades condicionais (4.9), seguindo os passos (i), (ii) e (iii) do algoritmo (3.3). 


\subsection{Mistura de Duas Distribuições Normais}

As distribuições normais com densidades (3.16), e as probabilidades (3.4), são çonsideradas no modelo (1.1). Assumindo independência à priori entre os parâmetros, consideramos as mesmas densidades à priori (3.17). A densidade à posteriori conjunta para $\Theta$ considerando a introdução das variáveis artificiais $W$ e $Z$ respectivamente, pode ser escrita como em (4.10). Portanto, podemos gerar amostras para $\alpha_{1}, \beta_{1}, \sigma_{1}, \alpha_{2}, \beta_{2}, \sigma_{2}$, $\gamma$, e $\tau$ à partir das densidades condicionais (4.11), seguindo os passos (i), (ii) e (iii) do algoritmo (3.3).

\subsection{Mistura das Distribuições Gamma-Normal}

Consideramos no modelo (1.1) uma mistura das distribuições gamma e normal com densidades (3.20) e probabilidades de incidência (3.4). Assumindo independência à priori entre os parâmetros as mesmas densidades à priori (3.21) novamente são consideradas.

Introduzindo as variáveis artificiais $W$ e $Z$ no modelo de mistura (1.1), temos que a densidade à posteriori conjuta de $\Theta$ pode ser expressa como em (4.12), e portanto podemos gerar amostras para $\alpha_{0}, \alpha_{1}, \beta_{1}, \alpha_{2}, \beta_{2}, \sigma$, $\gamma$, e $\tau$ à partira das densidades (4.13), seguindo os passos (i), (ii) e (iii) do algoritmo de Gibbs (3.3). 


\subsection{Algumas considerações na Seleção do Modelo}

Nesta dissertação, como apresentado nos capítulos 3,4 e 5, discutimos várias escolhas para as distribuições de latências condicionais no modelo de mistura (1.1), para os 3 diferentes esquemas amostrais (tempos observados completamente, censura tipo II, e censura por intervalos). Surge então a necessidade de determinar qual mistura dessas distribuições resulta em uma melhor modelagem dos tempos de sobrevivência $\mathbf{t}$.

$\mathrm{Na}$ literatura vários autores tem considerado uma aproximação Bayesiana da densidade preditiva baseado nos estimadores de Monte Carlo, para selecionar qual o melhor modelo, (ver por exemplo, Kuo e Peng , (1995); Gelfand, Dey e Chang (1992) ou Yang (1994)).

Consideramos neste trabalho a técnica das densidades preditivas condicionais ordenadas (CPO), tal técnica considera todos os tempos passados e futuros exceto o tempo presente $t_{i}$, onde a densidade preditiva condicionada é dada por:

$$
C_{i}=f\left(t_{i} \mid t_{(i)}, x_{i}\right)=\int f\left(t_{i} \mid \Theta, x_{i}\right) \pi\left(\Theta \mid t_{(i)}, x_{(i)}\right) d \Theta
$$

onde: $\pi\left(\Theta \mid t_{(i)}, x_{(i k)}\right)$ é a densidade à posteriori conjunta de $\Theta$, condicionada a todos os tempos $t_{1}, t_{2}, \ldots, t_{i-1}, t_{i+1}, \ldots, t_{n} ;$ e $f\left(t_{i} \mid \Theta, x_{i}\right)$ é definido em (1.1).

Podemos então, à partir das amostras geradas pelo algoritmo de Gibbs com Metropolis-Hastings, aproximar a densidade preditiva (5.2) através do estimador de Monte Carlo, assim, a densidade aproximada de (5.2) é dada 
por:

$$
\widehat{C}_{i}=f\left(t_{i} \mid \widehat{t_{(i)}}, x_{i}\right)=\frac{2}{R S} \sum_{r=1}^{R} \sum_{s=\frac{S}{2}+1}^{S} f\left(t_{i} \mid x_{i}, \Theta^{(r, s)}\right)
$$

onde $\Theta^{(r, s)}$ são as amostras geradas para as $S$ iterações em cada uma das $R$ cadeias, considerando diferentes valores iniciais para $\Theta$.

Portanto, podemos fazer para cada uma das misturas propostas nos 3 diferentes esquemas amostrais, um gráfico de $\widehat{C}_{i}$ versus $i, i=1, \ldots, n$, e o melhor modelo é o que apresentar em média, valores maiores de $\widehat{C}_{i}$. Podemos também usar o critério da verossimilhança preditiva, o qual seleciona o modelo que maximiza a verossimilhança $C(l)=\prod_{i=1}^{n} \widehat{C}_{i}(l)$, onde $l$ é o indice do $l$-ésimo modelo de mistura. Neste trabalho para cada um dos 3 esquemas amostrais temos, $l=1,2,3$, onde:

- $l=1$ : Modelo de Mistura de duas Distribuições Normais;

- $l=2$ : Modelo de Mistura das Distribuições Normal e Exponencial;

- $l=3$ : Modelo de Mistura das Distribuições Gamma e Normal. 


\section{Capítulo 6}

\section{Exemplos de Aplicações}

Neste capítulo, apresentaremos um exemplo numérico para cada uma das 3 diferentes estrutura de dados (sem censura, censura tipo II e censura por intervalo), para que possamos ilustrar a metodologia proposta neste trabalho.

No caso de dados observados completamente (iid) e dados com censura tipo II (ver capítulo 3 e 4), consideramos tempos de sobrevivência fictícios gerados à partir de Hewlett (1974), o qual considera os tempos de vida de 317 insetos do tipo (tribolium castaneum) dos quais 144, 69, $54 \mathrm{e}$ 50 consideramos como expostos à 4 diferentes concentrações de um inseticida chamado (pyrethrum) $0.20,0.32,0.50$ e $0.80 \mathrm{mg} / \mathrm{cm}^{2}$ respectivamente. Utilizamos o logaritmo das concentrações $-1.61,-1.14,-0.69$ e -0.22 respectivamente. Agora, para uma estrutura de dados censurados por intervalos (dados agrupados), consideramos os tempos de sobrevivência reais dos 317 insetos estudados por Hewlett (1974). 


\subsection{Exemplo 1: Dados Observados Completamente}

Na tabela (6.1), temos os tempos de sobrevivência de $n=317$ insetos gerados à partir de Hewlett (1974), dos quais 144, 69, 54 e 50 consideramos como expostos às concentrações de $0.20,0.30,0.50$ e $0.80 \mathrm{mg} / \mathrm{cm}^{2} \mathrm{de} \mathrm{um}$ certo inseticida, respectivamente.

Tabela 6.1: Tempos de Sobrevivência (em horas) de $n=317$ insetos expostos à 4 diferentes dosagens de um inseticida chamado (pyrethrum), considerando o logaritmo das doses

\begin{tabular}{cl}
\hline Log-Dose $(x)$ & \multicolumn{1}{c}{ Tempos de Sobrevivência $(\mathrm{t})$} \\
\hline$x_{1}=-1.61$ & $12,2(16), 5(30), 4(36), 2(40), 3(52), 2(60), 4(65), 70,2(76)$, \\
& $2(80), 3(90), 2(100), 2(110), 130,2(140), 150,160,180,280$ \\
& $300,20(400), 30(500), 15(700), 6(900)$ \\
\hline$x_{2}=-1.14$ & $3(10), 2(16), 2(20), 3(30), 3(35), 2(40), 2(45), 4(50), 3(56)$, \\
& $2(60), 2(65), 5(80), 3(85), 4(90), 4(92), 2(100), 115,130$, \\
& $160,340,5(400), 5(500), 4(580), 3(600), 2(800)$ \\
\hline$x_{3}=-0.69$ & $2(10), 2(18), 20,3(30), 2(32), 2(40), 45,4(50), 3(60), 2(65), 2(68)$ \\
& $5(80), 5(85), 5(85), 3(90), 2(92), 2(100), 2(118), 130,140,160,180$ \\
& $340,400,3(500), 580,650$ \\
\hline$x_{4}=-0.22$ & $2(10), 2(18), 3(30), 3(38), 2(40), 2(45), 2(50), 2(60), 3(68)$, \\
& $70,4(80), 5(86), 4(88), 90,3(100), 3(110), 2(118), 130,138$ \\
& $160,220,350,400$
\end{tabular}

Na figura (6.1), temos os histogramas de todos os tempos de sobrevivência (a), e para cada uma das 4 dosagens $x_{i k}, i=1, \ldots, n, k=$ $1,2,3,4$. Observamos claramente nestes gráficos uma distribuição bimodal, indicando a necessidade do uso da mistura de distribuições no modelo (1.1), e também observamos que em ambos os grupos o tempo de vida segue uma distribuição aproximadamente simétrica. 
(a) Todos os Dados

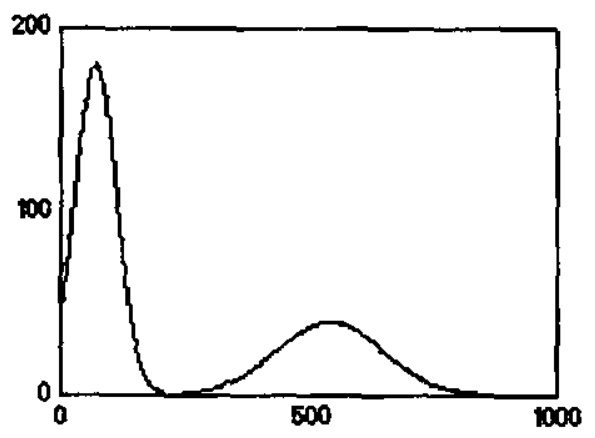

$x_{2}=-1.19$

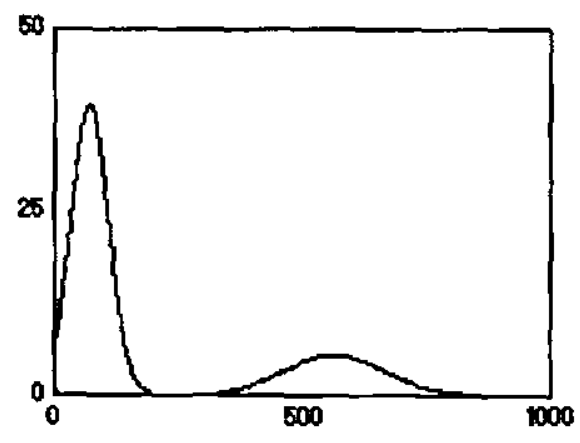

$x_{1}=-1.61$

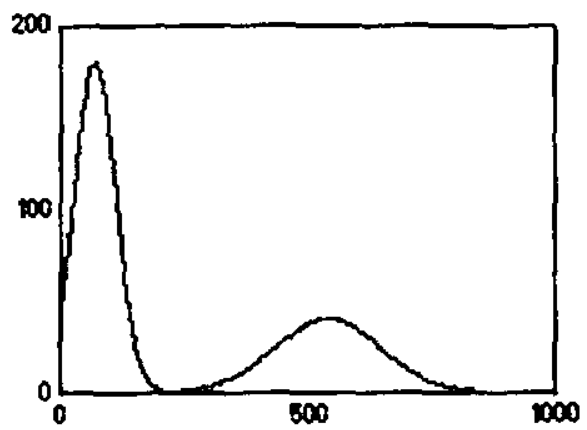

$x_{s}=-0.69$

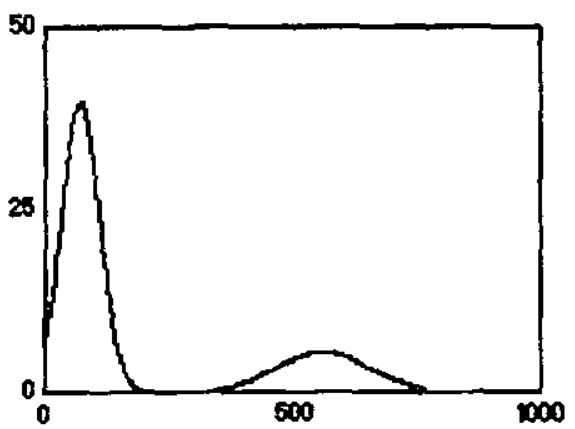

$x_{4}=-0.22$

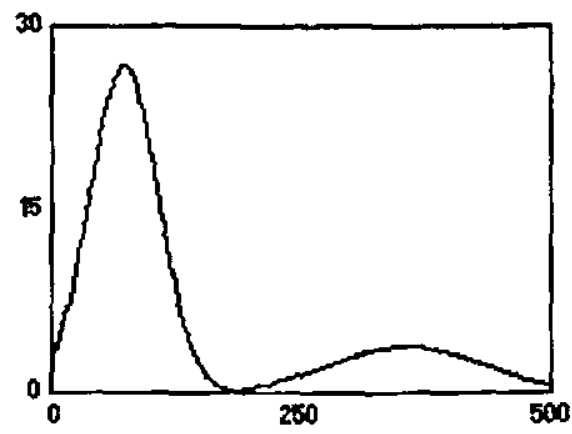

Figura 6.1: Histogramas dos tempos de sobrevivência da tabela (6.1), considerando todos os dados e cada uma das concentrações 


\subsubsection{Mistura de Duas Distribuiçōes Potência Exponencial}

Para analisarmos os tempos de sobrevivência da tabela (6.1), inicialmente consideramos a mistura de duas distribuições potência exponencial com densidades (1.4) onde $\theta_{j}=\alpha_{j}+\beta_{j} x_{i k}, j=1,2$, no modelo de mistura (1.1), e as probabilidades de incidência (3.4).Consideramos também as densidades à priori para $\alpha_{1}, \beta_{1}, \sigma_{1}, \delta_{1}, \alpha_{2}, \beta_{2}, \sigma_{2}, \delta_{2}, \gamma$ e $\tau$ dadas em (3.5) $\operatorname{com} \alpha_{10}=65.4, \sigma_{11}^{2}=14.1, \beta_{10}=-3.5, \sigma_{12}^{2}=7.7, \delta_{10}=0, \sigma_{13}^{2}=$ $0.01, \delta_{20}=0, \sigma_{23}^{2}=0.01, m_{11}=180, n_{11}=5.1, m_{22}=137, n_{22}=1.0, \alpha_{20}=$ $351.3, \sigma_{21}^{2}=3611.9, \beta_{20}=-126.8, \sigma_{22}^{2}=1573.4, \gamma_{0}=4.0, \sigma_{14}^{2}=2.5, \tau_{0}=$ 3.0 e $\sigma_{24}^{2}=2.3$ ( a escolha desses valores para os parâmetros das densidades à priori, foram baseados numa opinião experiente combinada com uma análise preliminar dos dados). A partir das densidades condicionais (3.11), seguindo os passos (ii) e (iii) do algoritmo de Gibbs (3.3), geramos para cada um dos parâmetros do modelo (1.1) 3 cadeias separadas de Gibbs cada uma com 15000 iterações, e devido ao fato de todas as densidades condicionais marginais não apresentarem uma forma padrão conhecida, utilizamos então o algoritmo Metropolis-Hastings (ver Apêndice B).

Para verificarmos a independência entre as iterações das amostras geradas, construímos um gráfico de autocorrelação dos 45000 pontos amostrais para cada um dos parâmetros $\alpha_{1}, \beta_{1}, \sigma_{1}, \delta_{1}, \alpha_{2}, \beta_{2}, \sigma_{2}, \delta_{2}, \gamma$ e $\tau$, veja figura (6.2). 

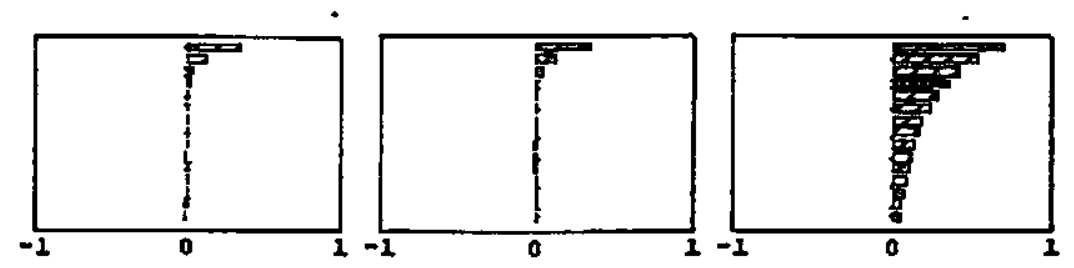

$\approx 1$

$\boldsymbol{\beta}_{1}$

$\omega_{1}$
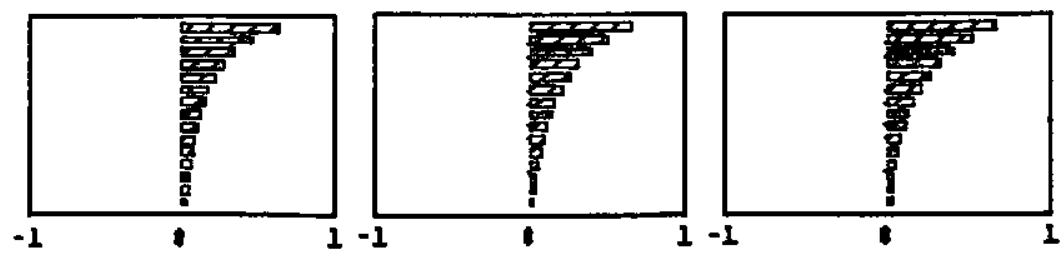

$\delta_{1}$

$a_{p}$

$\boldsymbol{\beta}_{2}$
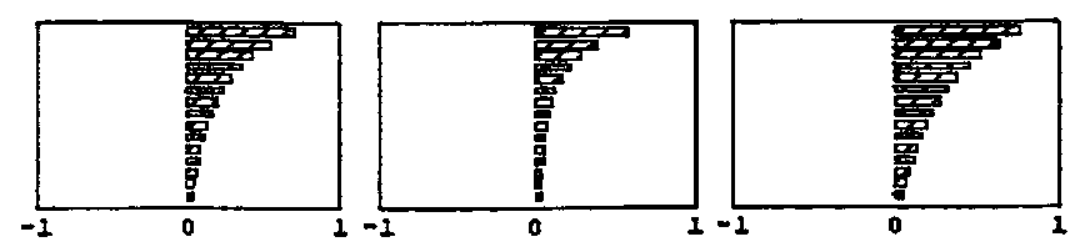

$\sigma_{2}$

$\delta_{2}$

$\boldsymbol{\gamma}$

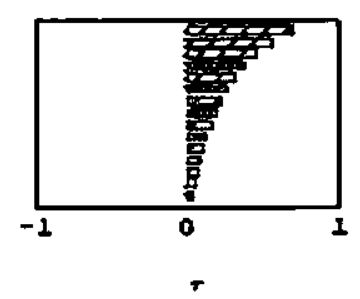

Figura 6.2: Correlação das amostras geradas pelo algoritmo Metropolis-Hastings, considerando os 45000 pontos amostrais

Como podemos observar na figura (6.2), temos um alto nível de correlação entre as iterações das amostras geradas à partir das densidades condicionais marginais (3.11), uma alternativa é considerar uma seleção mais refinada dessas' amostras. Portanto, para cada um dos parâmetros 
consideramos as iterações $15^{\underline{a}}, 30^{\underline{a}}, 45^{\underline{a}}, \ldots$, totalizando assim uma amostra final com $S=3000$ pontos. Com isso, conseguimos a independência entre os pontos amostrais, veja figura (6.3).

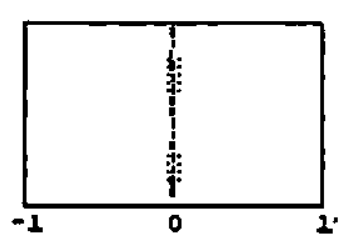

$a_{1}$

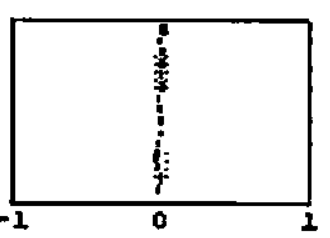

$\delta_{1}$

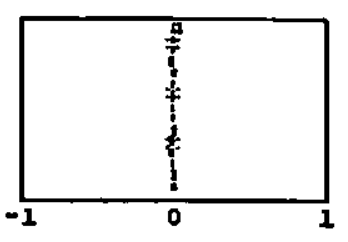

$\infty$

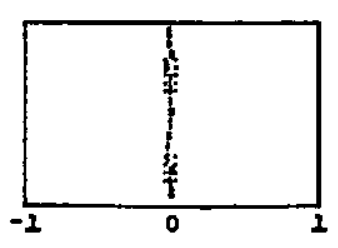

$\boldsymbol{\beta}_{1}$

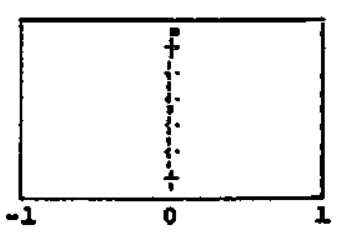

$a_{2}$

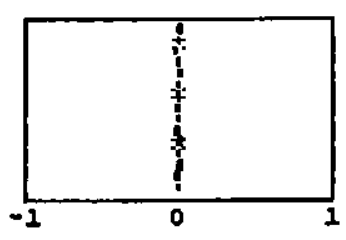

$\boldsymbol{\delta}_{2}$

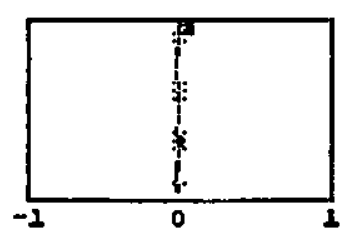

$\sigma_{1}$

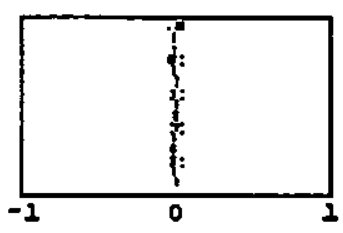

$\boldsymbol{\beta}_{2}$

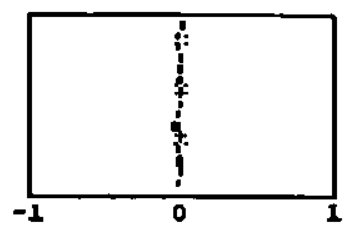

$\boldsymbol{\gamma}$

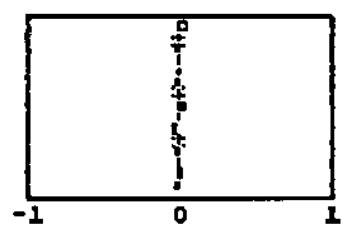

Figura 6.3: Correlação das amostras geradas pelo algoritmo Metropolis-Hastings, considerando os 3000 pontos amostrais selecionados de 15 em 15 da amostra total 
A convergência das amostras geradas pelo algoritmo Gibbs com Metropolis-Hastings foi monitorada utilizando o método proposto por Gelman e Rubin (1992) o qual se basea na técnica de análise de variância (ver Apêndice $\mathrm{C}$ ). Foram gastos 7 horas de trabalho computacional para gerar os 45000 pontos para cada um dos parâmetros $\alpha_{1}, \beta_{1}, \sigma_{1}, \delta_{1}, \alpha_{2}, \beta_{2}, \sigma_{2}, \delta_{2}$, $\gamma$ e $\tau$, utilizando o software S.A.S em um Pentium $166 \mathrm{MHZ}$.

Podemos observar na tabela (6.2), as médias à posteriori, intervalos de credibilidade de $95 \%$ e os estimadores dos fatores de redução de escala potencial $\widehat{R}$ (ver por exemplo, Gelman e Rubin, 1992), obtidos à partir das amostras de Gibbs geradas e selecionadas para cada um dos parâmetros.

Tabela 6.2: Quantidades à posteriori de interesse, obtidas à partir das amostras selecionadas, considerando a mistura de duas distribuições potência exponencial para o caso de dados observados completamente

\begin{tabular}{llll}
\hline Parâmetros & Média & Intervalo Credibilidade de $95 \%$ & $\hat{R}$ \\
\hline$\alpha_{1}$ & 66.65000 & $(60.42 ; 72.61)$ & 1.000711 \\
$\beta_{1}$ & -3.99000 & $(-8.87 ;-0.23)$ & 1.000117 \\
$\sigma_{1}$ & 38.21000 & $(32.74 ; 42.83)$ & 1.003883 \\
$\delta_{1}$ & 0.00870 & $(-0.00106 ; 0.028)$ & 1.000158 \\
$\alpha_{2}$ & 337.73000 & $(249.16 ; 429.11)$ & 1.001783 \\
$\beta_{2}$ & -124.12000 & $(-182.2 ;-64.82)$ & 1.001530 \\
$\sigma_{2}$ & 148.84000 & $(125.66 ; 169.58)$ & 1.000429 \\
$\delta_{2}$ & 0.00710 & $(-0.0123 ; 0.027)$ & 1.000107 \\
$\gamma$ & 4.02000 & $(1.53 ; 6.41)$ & 1.001148 \\
$\tau$ & 3.11000 & $(1.04 ; 5.20)$ & 1.001449 \\
\hline
\end{tabular}

Observamos na tabela (3.11), que $\sqrt{\widehat{R}}$ são bem menores que 1.1 , o que nos indica que o algoritmo de Gibbs com Metropolis-Hastings geraram para cada parâmetro amostras que convergem em distribuição para as 
distribuições condicionais marginais (3.11).

As densidades à posteriori marginais aproximadas considerando os $S=3000$ pontos amostrais são apresentadas na figura (6.4).

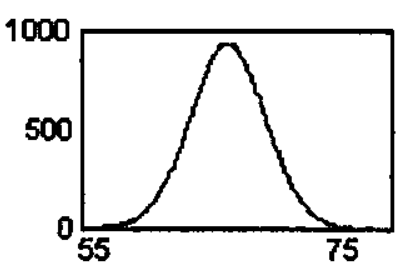

$\alpha_{1}$

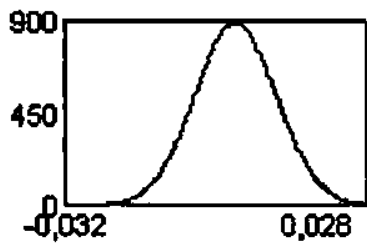

$\boldsymbol{\delta}_{\mathbf{1}}$

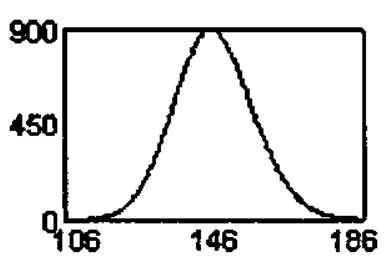

$\sigma_{2}$

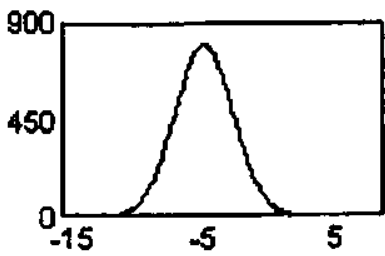

$\boldsymbol{\beta}_{1}$

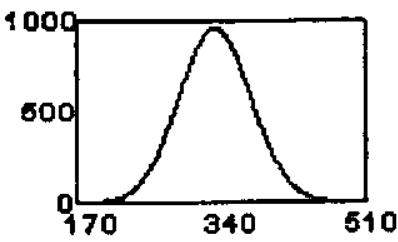

a

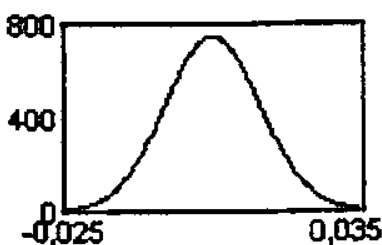

$\boldsymbol{z}_{2}$

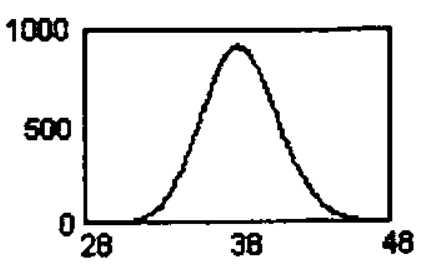

$\sigma_{1}$

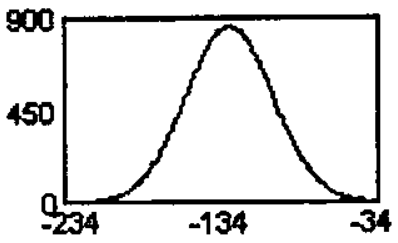

$\beta_{2}$

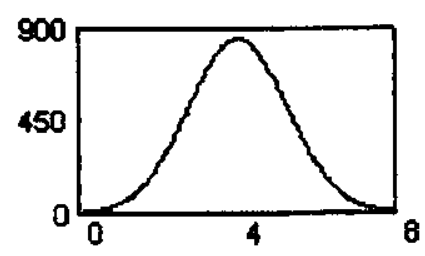

$\gamma$

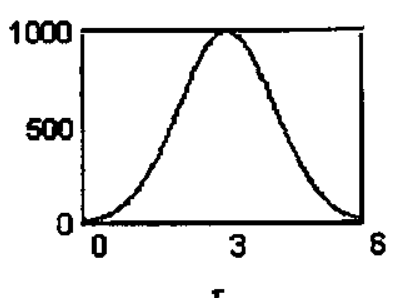

Figura 6.4: Densidades à posteriori marginais aproximadas considerando a mistura de duas distribuições potência exponencial, para o caso de dados sem censura 
À partir dos resultados da tabela (6.2), podemos considerar uma mistura de duas distribuições Normais no modelo de mistura (1.1) para analisar os dados da tabela (6.1), visto que os estimadores de Bayes para $\delta_{1}$ e $\delta_{2}$ baseados na aproximação de Monte Carlo para as médias à posteriori estão bem próximos de zero (observamos também que ambos os intervalos de credibilidade de $95 \%$ para $\delta_{1}$ e $\delta_{2}$ incluem o zero), quando $\delta_{j}=0$ a distribuição potência exponencial com densidade (1.4) assume a forma de uma distribuição normal, veja capítulo 1 seção 1.2 .

\subsubsection{Mistura de Duas Distribuições Normais}

Consideramos então uma mistura de duas distribuições normais com densidades (3.16) no modelo (1.1) e as densidades à priori (3.17) para os parâmetros com $\gamma_{0}=4.0, \sigma_{14}^{2}=0.60, \tau_{0}=3.0$ e $\sigma_{24}^{2}=0.4$. Geramos 3 cadeias separadas de Gibbs cada uma com 26000 iterações à partir das densidades condicionais marginais (3.19), seguindo os passos (ii) e (iii) do algoritmo de Gibbs (3.3), para gerar $\gamma$ e $\tau$ utilizamos o algoritmo Metropolis-Hastings por estes não apresentarem uma forma padrão conhecida. Selecionamos as amostras considerando as iterações $26^{\underline{a}}, 52^{\underline{a}}$, $78^{\underline{a}}, \ldots$, totalizando assim uma amostra final de $S=3000$ pontos (iid) para cada um dos parâmetros.

As quantidades à posteriori de interesses obtidas à partir das amostras selecionadas podemos verificar na tabela (6.3), onde observamos que os fatores de redução de escala potencial estão bem próximos de $1.1(\sqrt{\widehat{R}}<$ 1.1), indicando a convergência das amostras geradas. 
Tabela 6.3: Quantidades à posteriori de interesse, obtidas à partir das amostras selecionadas, considerando a mistura de duas distribuições normais, para o caso de dados observados completamente

\begin{tabular}{llll}
\hline Parâmetros & Média & Intervalo Credibilidade de $95 \%$ & $\widehat{R}$ \\
\hline$\alpha_{1}$ & 65.447 & $(57.248 ; 72.217)$ & 1.00006700 \\
$\beta_{1}$ & -3.465 & $(-10.590 ;-0.142)$ & 1.00002900 \\
$\sigma_{1}$ & 36.181 & $(31.993 ; 40.891)$ & 1.00246600 \\
$\alpha_{2}$ & 351.260 & $(235.177 ; 468.857)$ & 1.00353600 \\
$\beta_{2}$ & -126.806 & $(-203.062 ;-50.101)$ & 1.00398100 \\
$\sigma_{2}$ & 135.419 & $(117.943 ; 158.697)$ & 1.00196700 \\
$\gamma$ & 4.020 & $(2.620 ; 5.422)$ & 1.00001800 \\
$r$ & 3.021 & $(1.923 ; 4.085)$ & 1.00342600 \\
\hline
\end{tabular}

Agora na figura (6.5) temos as densidades à posteriori marginais aproximadas considerando os $S=3000$ pontos amostrais selecionados.

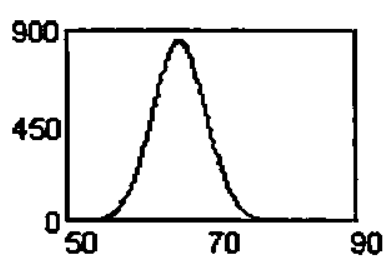

$\mathbf{a}_{1}$

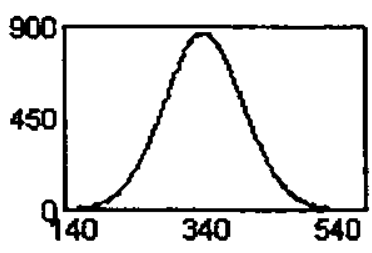

$a_{2}$

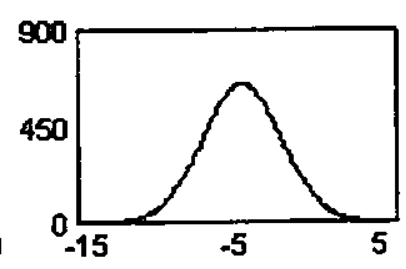

$\beta_{1}$

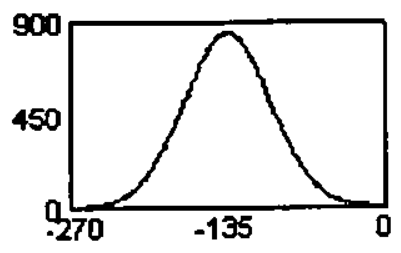

$\boldsymbol{\beta}_{2}$

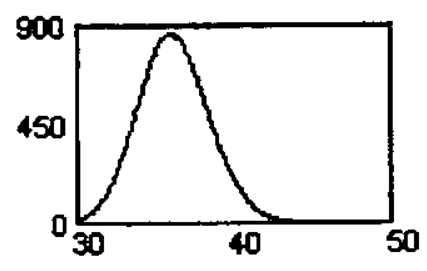

$\sigma_{1}$

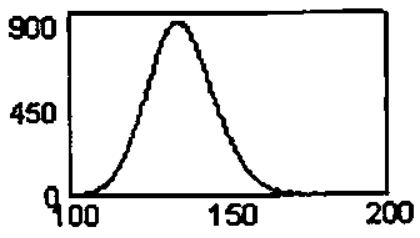

$\sigma_{1}$ 

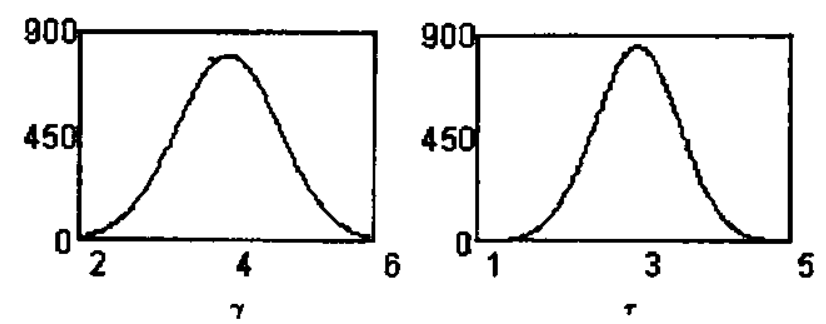

Figura 6.5: Densidades à posteriori marginais aproximadas considerando a mistura de duas distribuições normais, para o caso de dados sem censura

Observamos que as inferências obtidas para os parâmetros $\alpha_{1}, \beta_{1}, \sigma_{1}$, $\alpha_{2}, \beta_{2}, \sigma_{2}, \gamma$ e $\tau$, são bastante similares considerando ambas as misturas (6.1.1) e (6.1.2) . Portanto é razoável considerar a mistura de duas distribuições normais no modelo (1.1) para analisar os dados da tabela (6.1).

Entretando consideramos ainda no modelo (1.1) a mistura das distribuições normal-exponencial e gamma-normal, até mesmo para tentar comprovar a eficiência da distribuição potência exponencial.

\subsubsection{Mistura das Distribuiçōes Normal-Exponencial}

Para analisar os dados da tabela (6.1), consideramos agora a mistura das distribuições normal-exponencial com densidades (3.12) no modelo de mistura (1.1), com as probabilidades de incidência dadas por (3.4) e as densidades à priori (3.13) para os parâmetros, com $\gamma_{0}=4, s_{1}^{2}=1.1$, $\tau_{0}=3.5$ e $s_{2}^{2}=0.9$.

A partir das densidades condicionais marginais (3.15) seguindo os passos (ii) e (iii) do algoritmo de Gibbs (3.3), geramos 3 cadeias separadas de Gibbs cada uma com 26000 iterações e utilizamos o método proposto por Gelman e Rubin (1992) para verificar a convergência das amostras. 
Para cada um dos parâmetros consideramos as iterações $26^{\underline{a}}, 52^{\underline{a}}, 78^{\underline{a}}, \ldots$, totalizando assim uma amostra final de $S=3000$ pontos amostrais iid. Para gerar os parâmetros $\alpha_{2}, \beta_{2}, \gamma$ e $\tau$, utilizamos o algoritmo MetropolisHastings, por estes não apresentarem uma forma padrão conhecida.

$\mathrm{Na}$ tabela (6.4) temos as quantidades à posteriori de interesse, médias à posteriori, intervalos de credibilidades de $95 \%$ e os estimadores dos fatores de redução de escala potencial $\widehat{R}$.

Tabela 6.4: Quantidades à posteriori de interesse, obtidas à partir das amostras selecionadas, considerando a mistura das distribuições normal-exponencial, para o caso de dados observados completamente

\begin{tabular}{llll}
\hline Parâmetros & Média & Intervalo Credibilidade de $95 \%$ & $\hat{R}$ \\
\hline$\alpha_{1}$ & 63.810 & $(55.94 ; 71.23)$ & 1.00008700 \\
$\beta_{1}$ & -4.340 & $(-13.24 ;-0.17)$ & 1.00000000 \\
$\sigma$ & 32.280 & $(27.53 ; 37.27)$ & 1.00112400 \\
$\alpha_{2}$ & 0.0037 & $(0.003 ; 0.0045)$ & 1.00211000 \\
$\beta_{2}$ & 0.0003 & $(0.0003 ; 0.00043)$ & 1.00000000 \\
$\gamma$ & 4.04 & $(2.38 ; 5.59)$ & 1.00015000 \\
$\tau$ & 3.61 & $(1.58 ; 5.65)$ & 1.00629900 \\
\hline
\end{tabular}

Podemos observar que $\sqrt{\widehat{R}}<1.1$ para todos os parâmetros, o que nos garante que as amostras selecionadas convergem em distribuição para as suas respectivas densidades à posteriori marginais em (3.15).

\subsubsection{Mistura das Distribuiçōes Gamma-Normal}

Finalmente consideramos no modelo (1.1) uma mistura das distribuições gamma e normal com densidades (3.20), as probabilidades de in- 
cidência (3.4) e as densidades à priori (3.21) para os parâmetros, com $\gamma_{0}=4, s_{1}^{2}=0.1, \tau_{0}=3$ e $s_{2}^{2}=0.09$.

Seguindo os passos (ii) e (iii) do algoritmo de Gibbs (3.3), geramos 3 cadeias separadas de Gibbs cada uma com 26000 iterações à partir das densidades à posteriori marginais (3.23). Selecionamos um amostra iid considerando as iterações $26^{\underline{a}}, 52^{\underline{a}}, 78^{\underline{a}}, \ldots$, e utilizamos o critério de Gelman e Rubin (1992) para verificar a convergência das amostras selecionadas.

As médias à posteriori, intervalos de credibilidade de $95 \%$ e o fator de redução de escala potencial $\widehat{R}$ calculados à partir das amostras de Gibbs selecionadas, podem ser observados na tabela (6.5).

Tabela 6.5: Quantidades à posteriori de interesse, obtidas à partir das amostras selecionadas, considerando a mistura das distribuições gamma-normal, para o caso de dados observados completamente

\begin{tabular}{llll}
\hline Parâmetros & Média & Intervalo Credibilidade de $95 \%$ & $\widehat{R}$ \\
\hline$\alpha_{0}$ & 1.05 & $(0.921 ; 1.216)$ & 1.00174800 \\
$\alpha_{1}$ & 0.016 & $(0.0128 ; 0.0204)$ & 1.00632700 \\
$\beta_{1}$ & 0.002 & $(0.0006 ; 0.004)$ & 1.00305900 \\
$\alpha_{2}$ & 342.53 & $(144.00 ; 508.23)$ & 1.00416400 \\
$\beta_{2}$ & -130.60 & $(-248.68 ;-22.78)$ & 1.00466600 \\
$\sigma$ & 140.62 & $(116.80 ; 180.98)$ & 1.00111200 \\
$\gamma$ & 4.00 & $(3.46 ; 4.58)$ & 1.00022700 \\
$\tau$ & 3.00 & $(2.44 ; 3.61)$ & 1.00119100 \\
\hline
\end{tabular}

Podemos observar que para todos os parâmetros, temos $\sqrt{\widehat{R}}<1.1$ indicando a convergência das amostras geradas. 


\subsubsection{Discriminação dos Modelos}

Para selecionar dentre os modelos de misturas (6.1.2), (6.1.3) e (6.1.4), o que se ajusta melhor aos dados da tabela (6.1), utilizamos a técnica das densidades preditivas condicionais ordenadas (CPO), veja o capítulo 5 seção 5.5. Para isso, consideramos à partir das (CPO) o critério da verossimilhança preditiva o qual seleciona o melhor modelo aquele que maximiza a verossimilhança $C(l)=\prod_{i=1}^{n} \widehat{C}_{i}(l)$, onde $l$ é o indice do $l$-ésimo modelo de mistura, veja tabela (6.6).

Tabela 6.6: Densidades Preditivas Condicionais Ordenadas e o Produto da Verossimilhança Preditiva $C(l)=\prod_{i=1}^{n} \widehat{C}_{i}(l)$ para cada um dos modelos de mistura (6.1.2), (6.1.3) e (6.1.4)

\begin{tabular}{|c||c|c|c|}
\hline \multicolumn{4}{|c|}{ Densidades Preditivas Condicionais Ordenadas } \\
\hline \hline & Modelo (6.1.2) & Modelo (6.1.3) & Modelo (6.1.4) \\
\hline $\mathrm{i}$ & $\widehat{C}_{i}(1)$ & $\widehat{C}_{i}(2)$ & $\widehat{C}_{i}(3)$ \\
\hline \hline 1 & 0.0024217653 & 0.0037150198 & 0.0032933737 \\
2 & 0.0028783722 & 0.0039881063 & 0.0031656465 \\
3 & 0.0028783722 & 0.0039881063 & 0.0031656465 \\
4 & 0.0047982638 & 0.005258899 & 0.0027127556 \\
5 & 0.0047982638 & 0.005258899 & 0.0027127556 \\
$\vdots$ & $\vdots$ & $\vdots$ & $\vdots$ \\
317 & 0.0002850037 & 0.0000487115 & 0.0002603672 \\
\hline \hline$\prod_{i=1}^{n} \widehat{C}_{i}(l)$ & $3.14 \times 100^{-390}$ & $2.56 \times 100^{-501}$ & $2.11 \times 100^{-478}$ \\
\hline
\end{tabular}

Observamos na tabela (6.6) que o modelo de mistura de duas distribuições Normais (6.1.2) pode ser considerado o melhor modelo para os dados da tabela (6.1), pelo fato de ter apresentado o valor máximo do pro- 
duto da verossimilhança $\prod_{i=1}^{n} \widehat{C}_{i}(l)$, o que vem sustentar ainda mais a grande importância do uso das distribuições potência exponencial com densidade (1.4), para indicar que distribuição padrão conhecida pode ser usada no modelo de mistura (1.1), e portanto não precisamos nos basear apenas em uma análise preliminar dos dados para estas escolhas.

\subsubsection{Análise Clássica}

Em se tratando da teoria clássica encontramos os estimadores de máxima verossimilhança assintóticos (E.M.V.) para os modelos (6.1.2), (6.1.3) e (6.1.4), utilizando o método numérico de Newton-Raphson, mas não conseguimos obter estes estimadores para o caso do modelo (6.1.1), devido a sua maior complexidade, veja tabela (6.7).

Podemos observar à partir da tabela (6.7) que os valores dos E.M.V. para o parâmetrò $\beta_{1}$ no caso dos modelos de mistura (6.1.2) e (6.1.3) e para os parâmetros $\alpha_{0}, \beta_{1}, \gamma$ e $\tau$ no caso da mistura (6.1.4), são um pouco diferentes dos obtidos pelos métodos Bayesianos.

Dos intervalos de credibilidade de $95 \%$ para $\beta$ nas tabelas (6.2), (6.4) e (6.5), observamos que há um efeito muito pequeno das dosagens do inseticida no tempo de vida do grupo mais resistente ao tratamento. Este resultado está de acordo com o estudo anterior de Hew̉lett (1974) e de Kuo e Peng (1995) considerando tempos de sobrevivência censurados por intervalos.

Observamos também à partir dos intervalos de credibilidade de $95 \%$ para $\tau$ nas tabelas $(6.2),(6.4)$ e (6.5), que há um grande efeito dos níveis 
Tabela 6.7: Estimadores de Máxima Verossimilhança e intervalos de confiança assintóticos de $95 \%$ dos parâmetros dos modelos (6.1.2), (6.1.3) e (6.1.4), para o caso de dados observados completamente

\begin{tabular}{|c|c|c|c|c|c|c|}
\hline \multicolumn{7}{|c|}{ Inferência Assintótica } \\
\hline Par. & \multicolumn{2}{|c|}{ Modelo (6.1.2) } & \multicolumn{2}{c|}{ Modelo (6.1.3) } & \multicolumn{2}{c|}{ Modelo (6.1.4) } \\
\hline \hline & E.M.V. & I.C.de 95\% & E.M.V. & I.C.de 95\% & E.M.V. & I.C.de 95\% \\
\hline \hline$\alpha_{0}$ & - & - & - & - & 1.30 & $(1.24 ; 1.37)$ \\
$\alpha_{1}$ & 72.22 & $(68.38 ; 76.06)$ & 74.17 & $(72.63 ; 75.72$ & 0.016 & $(0.015 ; 0.017)$ \\
$\beta_{1}$ & 3.97 & $(0.14 ; 7.80)$ & 8.97 & $(7.31 ; 10.63)$ & 0.008 & $(0.0075 ; 0.009)$ \\
$\sigma$ & - & - & 32.73 & $(31.58 ; 33.88)$ & 342.16 & $(339.42 ; 344.89)$ \\
$\sigma_{1}$ & 35.96 & $(34.57 ; 37.35)$ & - & - & - & - \\
$\alpha_{2}$ & 359.80 & $(354.53 ; 365.04)$ & 0.003 & $(0.0025 ; 0.004)$ & -129.81 & $(-131.68 ;-127.93)$ \\
$\beta_{2}$ & -121.92 & $(-130.18 ;-113.65)$ & 0.0007 & $(0.0003 ; 0.001)$ & 140.26 & $(137.46 ; 143.06)$ \\
$\sigma_{2}$ & 133.16 & $(128.05 ; 138.27)$ & - & - & - & - \\
$\gamma$ & 4.03 & $(3.67 ; 4.38)$ & 3.94 & $(3.53 ; 4.35)$ & 8.34 & $(7.64 ; 9.04)$ \\
$\tau$ & 3.02 & $(2.76 ; 3.28)$ & 3.24 & $(2.93 ; 3.54)$ & -1.28 & $(-1.49 ;-1.08)$ \\
\hline \hline
\end{tabular}

do inseticida $x_{i k}$, nas probabilidades de incidência das populações.

\subsection{Exemplo 2: Dados com Censura Tipo II}

Neste exemplo consideramos também tempos de sobrevivência de $n=$ 317 insetos gerados à partir de Hewlett (1974), assumindo agora uma estrutura de dados com censura à direita de $t_{i h}$ (censura tipo II). Dentre os $n=317$ insetos $144,69,54$ e 50 consideramos como sendo expostos à 4 diferentes concentrações de um inseticida, $0.20,0.32,0.50$ e $0.80 \mathrm{mg} / \mathrm{cm}^{2}$, respectivamente. Utilizamos o logaritmo das concentrações $-1.61,-1.14$, -0.69 e - 0.22 . Para cada nível da covariável $x_{i k}, i=1, \ldots, n_{i} k=1,2,3,4$, o experimento termina quando observamos $r_{1}=38, r_{2}=33, r_{3}=34 \mathrm{e}$ 
88 Exemplos de Aplicações Capítulo 6

$r_{4}=35$ mortes, respectivamente.

Tabela 6.8: Tempos de Sobrevivência (em horas) de $n=317$ insetos expostos à 4 diferentes dosagens de um inseticida chamado (pyrethrum), considerando o logaritmo das doses

\begin{tabular}{ll}
\hline \multicolumn{1}{c}{ Log-Dosage $(x)$} & \multicolumn{1}{c}{ Survival Times $(\mathrm{t})$} \\
\hline$x_{1}=-1.61$ & $12,2(16), 5(30), 4(36), 2(40), 3(52), 2(60), 4(65), 70,2(76)$, \\
$n_{1}=144, r_{1}=38$ & $2(80), 3(90), 2(100), 2(110), 130,2(140), 106(140+)$ \\
\hline$x_{2}=-1.14$ & $3(10), 2(16), 2(20), 3(30), 3(35), 2(40), 2(45), 4(50), 3(56)$, \\
$n_{2}=69, r_{2}=33$ & $2(60), 2(65), 5(80), 36(80+)$ \\
\hline$x_{3}=-0.69$ & $2(10), 2(18), 20,3(30), 2(32), 2(40), 45,4(50), 3(60), 2(65)$, \\
$n_{3}=54, r_{3}=34$ & $2(68), 5(80), 5(85), 20(85+)$ \\
\hline$x_{4}=-0.22$ & $2(10), 2(18), 3(30), 3(38), 2(40), 2(45), 2(50), 2(60), 3(68)$, \\
$n_{4}=50, r_{4}=35$ & $70,4(80), 5(86), 4(88), 15(88+)$ \\
\hline
\end{tabular}

(Observaçōes com + são censuradas)

Na figura (6.6), temos a distribuição dos tempos de şobrevivência da tabela (6.8). Podemos constatar por este gráfico um comportamento bimodal e em ambos os grupos uma distribuição de frequência aproximadamente simétrica dos tempos de vida destes insetos, indicando claramente a necessidade do uso da mistura de distribuições mais especificamente o uso da mistura de $J=2$ componentes no modelo (1.1). 


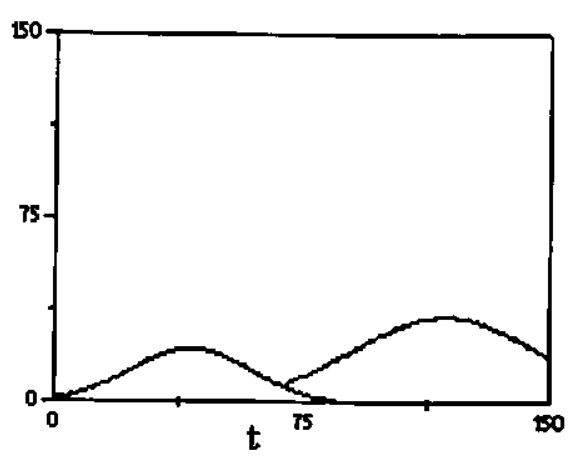

Figura 6.6: Histogramas dos tempos de sobrevivência da tabela (6.8)

\subsubsection{Mistura de Duas Distribuições Potência Exponencial}

Assumimos no modelo (1.1) a mistura de duas distribuições potência exponencial com densidades (1.4) onde $\theta_{j}=\left(\alpha_{j}+\beta_{j} x_{i k}\right), j=1,2$, as probabilidades de incidência (3.4) para analisarmos os tempos de sobrevivência da tabela (6.8). As densidades à priori (3.5) para $\alpha_{1}, \beta_{1}, \sigma_{1}, \delta_{1}$, $\alpha_{2}, \beta_{2}, \sigma_{2}, \delta_{2}, \gamma$ e $\tau$ foram consideradas assumindo $\alpha_{10}=57.85, \sigma_{11}^{2}=$ $142.76, \beta_{10}=-9.43, \sigma_{12}^{2}=76.35, \delta_{10}=0, \sigma_{13}^{2}=0.01, \delta_{20}=0, \sigma_{23}^{2}=$ $0.01, m_{11}=180, n_{11}=5.1, m_{22}=137, n_{22}=1.0, \alpha_{20}=95.77, \sigma_{21}^{2}=$ $3801.11, \beta_{20}=-414.92, \sigma_{22}^{2}=1394.21, \gamma_{0}=4.0, \sigma_{14}^{2}=0.7, \tau_{0}=3.6 \mathrm{e}$ $\sigma_{24}^{2}=1.1$ ( a escolha desses valores para os parâmetros das densidades à priori, foram baseados numa opinião experiente combinada com uma análise preliminar dos dados).

Utilizamos a função distribuição acumulada da distribuição potência exponencial definida em (1.6) para gerar a variável artificial $w_{i}$ no algoritmo EM à partir da densidade (2.4), veja capítulo 2 seção 2.2. Portanto, seguindo os passos (i)-(iii) do algoritmo de Gibbs (3.3), à partir das densidades condicionais marginais (4.7) geramos para cada parâmetro 3 cadeias 
separadas de Gibbs com 5000 iterações cada uma. Devido todas as densidades marginais em (4.7) não apresentarem uma forma padrão conhecida, utilizamos o algoritmo Metropolis-Hastings para gerar as amostras (ver Apêndice B), e monitoramos a convergência das amostras através do método proposto por Gelman e Rubin (1992) (ver Apêndice C). Selecionamos uma amostra iid considerando apenas as iterações $10^{a}, 20^{a}$, $30^{\underline{a}}, \ldots$, totalizando uma amostra final de $S=1500$ pontos iid para cada um dos parâmetros do modelo, dos quais calculamos algumas quantidades à posteriori de interesse como médias, intervalos de credibilidade de $95 \%$ e os fatores de redução de escala potencial $\widehat{R}$ (ver por exemplo Gelman e Rubin, 1992), as quais podemos verificar na tabela (6.9).

Tabela 6.9: Quantidades à posteriori de interesse, obtidas à partir das amostras selecionadas, considerando a mistura de duas distribuições potência exponencial para o caso de dados com censura tipo II

\begin{tabular}{llll}
\hline Parâmetros & Média & Intervalo Credibilidade de $95 \%$ & $\hat{R}$ \\
\hline$\alpha_{1}$ & 60.55 & $(39.16 ; 82.83)$ & 1.000210 \\
$\beta_{1}$ & -18.87 & $(-34.46 ;-2.55)$ & 1.002037 \\
$\sigma_{1}$ & 35.39 & $(30.44 ; 40.70)$ & 1.003240 \\
$\delta_{1}$ & 0.074 & $(-0.012 ; 0.026)$ & 1.000833 \\
$\alpha_{2}$ & 36.76 & $(0.72 ; 152.35)$ & 1.002680 \\
$\beta_{2}$ & -368.69 & $(-441.76 ;-310.72)$ & 1.003292 \\
$\sigma_{2}$ & 142.95 & $(117.17 ; 167.73)$ & 1.001941 \\
$\delta_{2}$ & 0.0033 & $(-0.016 ; 0.023)$ & 1.000914 \\
$\gamma$ & 4.39 & $(2.92 ; 5.90)$ & 1.000608 \\
$\tau$ & 2.95 & $(1.45 ; 4.51)$ & 1.000252 \\
\hline
\end{tabular}

A distribuição potência exponencial assume em sua forma (1.4) uma grande classe de distribuições simétricas, por exemplo quando $\delta_{j}=0$ a distribuição (1.4) assume a forma de uma distribuição normal, veja capítulo 
1 seção 1.2. Portanto à partir dos resultados da tabela (6.9) podemos assumir agora uma mistura de duas distribuições normais no modelo (1.1) para analisar os dados da tabela (6.8), uma vez que as médias à posteriori para $\delta_{1}$ e $\delta_{2}$ estão bem próximos de zero e seus respectivos intervalos de credibilidade de $95 \%$ incluem o zero.

\subsubsection{Mistura de Duas Distribuiçōes Normais}

Consideramos no modelo (1.1) a mistura de duas distribuições normais com densidades (3.16) e as probabilidades de incidência (3.4), assumindo independência à priori entre os parâmetros e as densidades à priori (3.17) com $\gamma_{0}=4.0, \sigma_{14}^{2}=0.70, \tau_{0}=3.6$ e $\sigma_{24}^{2}=1.1$. Geramos 3 cadeias separadas de Gibbs cada uma com 7000 iterações à partir das densidades condicionais marginais (4.11) seguindo os passos (i)-(iii) do algoritmo de Gibbs (3.3), com um tempo de trabalho computacional de aproximadamente 40 horas. Por não apresentarem uma forma padrão conhecida $\gamma \mathrm{e}$ $\tau$ foram gerados utilizando o algoritmo Metropolis-Hastings. Selecionamos as amostras considerando as iterações $10^{a}, 20^{a}, 30^{a}, \ldots$, totalizando assim uma amostra final de $S=2100$ pontos amostrais (iid) para cada um dos parâmetros.

As quantidades à posteriori de interesses obitidas à partir das amostras selecionadas podemos verificar na tabela (6.10), onde observamos que os fatores de redução de escala potencial estão bem próximos de $1.1(\sqrt{\widetilde{R}}<$ 1.1), indicando a convergência das amostras geradas.

Observamos que as inferências obtidas para os parâmetros $\alpha_{1}, \gamma$ e $\tau$, são bastante similares considerando ambas as misturas (6.2.1) e (6.2.2), 
Tabela 6.10: Quantidades à posteriori de interesse, obtidas à-partir das amostras selecionadas, considerando a mistura de duas distribuições normais, para o caso de dados com censura tipo II

\begin{tabular}{llll}
\hline Parâmetros & Média & Intervalo Credibilidade de $95 \%$ & $\widehat{R}$ \\
\hline$\alpha_{1}$ & 57.849 & $(35.481 ; 85.786)$ & 1.00096750 \\
$\beta_{1}$ & -9.426 & $(-32.184 ;-0.265)$ & 1.00512800 \\
$\sigma_{1}$ & 57.440 & $(25.610 ; 152.020)$ & 1.00057800 \\
$\alpha_{2}$ & 95.774 & $(5.756 ; 231.567)$ & 1.00293320 \\
$\beta_{2}$ & -414.919 & $(-479.140 ;-339.250)$ & 1.00080900 \\
$\sigma_{2}$ & 215.961 & $(154.395 ; 312.234)$ & 1.00232900 \\
$\gamma$ & 3.836 & $(2.538 ; 5.223)$ & 1.00071700 \\
$\tau$ & 3.270 & $(2.097 ; 4.742)$ & 1.00152600 \\
\hline
\end{tabular}

mas para $\beta_{1}, \sigma_{1}, \alpha_{2}, \beta_{2}, \sigma_{2}$, estas inferências são um pouco diferentes, talvez pelo valores diferentes atribuídos à esses parâmetros na densidade à priori (3.5). Portanto é razoável considerar a mistura de duas distribuições normais no modelo (1.1) para analisar os dados da tabela (6.8).

Entretando consideramos ainda no modelo (1.1) a mistura das distribuições normal-exponencial e gamma-normal, até mesmo para tentar comprovar a eficiência da distribuição potência exponencial.

\subsubsection{Mistura das Distribuições Normal-Exponencial}

Para analisar os dados da tabela (6.8), consideramos agora a mistura das distribuições normal-exponencial com densidades (3.12) no modelo de mistura (1.1), com as probabilidades de incidência dadas por (3.4) e as densidades à priori (3.13) para os parâmetros com $\gamma_{0}=4, s_{1}^{2}=0.7, \tau_{0}=3.6$ 
e $s_{2}^{2}=1.1$.

A partir das densidades condicionais marginais (4.9) e seguindo os passos (i)-(iii) do algoritmo de Gibbs (3.3), geramos 3 cadeias separadas de Gibbs cada uma com 7000 iterações e utilizamos o método proposto por Gelman e Rubin (1992) para verificar a convergência das amostras. Para cada um dos parâmetros consideramos as iterações $10^{\underline{a}}, 20^{\underline{a}}, 30^{\underline{a}}, \ldots$, totalizando assim uma amostra final de $S=2100$ pontos amostrais iid.

$\mathrm{Na}$ tabela (6.11) temos as quantidades a posteriori de interesse, médias à posteriori, intervalos de credibilidade $95 \%$ e os estimadores dos fatores de redução de escala potencial $\widehat{R}$.

Tabela 6.11: Quantidades à posteriori de interesse, obtidas à partir das amostras selecionadas, considerando a mistura das distribuições normal-exponencial, para o caso de dados com censura tipo II

\begin{tabular}{llll}
\hline Parâmetros & Média & Intervalo Credibilidade de $95 \%$ & $\hat{R}$ \\
\hline$\alpha_{1}$ & 62.66 & $(51.658 ; 73.514)$ & 1.00049530 \\
$\beta_{1}$ & -5.56 & $(-17.838 ;-0.216)$ & 1.00005074 \\
$\sigma$ & 37.78 & $(29.321 ; 50.367)$ & 1.00132800 \\
$\alpha_{2}$ & 0.005 & $(0.0033 ; 0.0057)$ & 1.00168700 \\
$\beta_{2}$ & 0.0004 & $(0.00027 ; 0.00053)$ & 1.00000000 \\
$\gamma$ & 3.96 & $(2.4550 ; 5.537)$ & 1.00482200 \\
$\tau$ & 3.65 & $(1.665 ; 5.679)$ & 1.00079900 \\
\hline
\end{tabular}

Observamos na tabela $(6.11)$ que $\sqrt{\widehat{R}}<1.1$ para todos os parâmetros, o que nos garante que as amostras selecionadas convergem em distribuição para as suas respectivas densidades à posteriori marginais em (4.9). 


\subsubsection{Mistura das Distribuições Gamma-Normal}

Assumimos agora no modelo (1.1) uma mistura das distribuições gamma-normal com densidades (3.20), as probabilidades de incidência (3.4) e as densidades à priori (3.21) para os parâmetros, com $\gamma_{0}=4, s_{1}^{2}=0.7$, $\tau_{0}=3.6$ e $s_{2}^{2}=1.1$.

Seguindo os passos (i)-(iii) do algoritmo de Gibbs (3.3), geramos 3 cadeias separadas de Gibbs cada uma com 7000 iterações à partir das densidades à posteriori marginais (4.13). Selecionamos uma amostra $i i d$ considerando as iterações $10^{\underline{a}}, 20^{\underline{a}}, 30^{\underline{a}}, \ldots$, e utilizamos o critério de Gelman e Rubin (1992) para verificar a convergência das amostras selecionadas.

As médias à posteriori, intervalos de credibilidade $95 \% \mathrm{e}$ os fatores de redução de escala potencial $\widehat{R}$ calculados à partir das amostras de Gibbs selecionadas, podem ser observados na tabela (6.12).

Tabela 6.12: Quantidades à posteriori de interesse, obtidas à partir das amostras selecionadas, considerando a mistura das distribuições gamma-normal, para o caso de dados com censura tipo II

\begin{tabular}{llll}
\hline Parâmetros & Média & Intervalo Credibilidade de $95 \%$ & $\widehat{R}$ \\
\hline$\alpha_{0}$ & 0.97 & $(0.834 ; 1.194)$ & 1.01019800 \\
$\alpha_{1}$ & 0.02 & $(0.013 ; 0.026)$ & 1.01630900 \\
$\beta_{1}$ & 0.003 & $(0.0007 ; 0.0042)$ & 1.02498200 \\
$\alpha_{2}$ & 31.13 & $(1.363 ; 97.343)$ & 1.00276200 \\
$\beta_{2}$ & -315.21 & $(-362.928 ;-214.515)$ & 1.00386600 \\
$\sigma$ & 147.07 & $(94.676 ; 234.364)$ & 1.00465200 \\
$\gamma$ & 4.05 & $(2.494 ; 5.706)$ & 1.00575100 \\
$\tau$ & 3.30 & $(1.568 ; 5.546)$ & 1.00582800 \\
\hline
\end{tabular}


Podemos observar que para todos os parâmetros, temos $\sqrt{\widehat{R}}<1.1$ indicando a convergência das amostras geradas.

\subsubsection{Discriminação dos Modelos}

Para selecionar dentre os modelos de misturas (6.2.2), (6.2.3) e (6.2.4), o que se ajusta melhor aos dados da tabela (6.8), utilizamos a técnica das densidades preditivas condicionais ordenadas (CPO), veja o capítulo 5 seção 5.5. Para isso, consideramos à partir das (CPO) o critério da verossimilhança preditiva o qual seleciona o melhor modelo aquele que maximiza a verossimilhança $C(l)=\prod_{i=1}^{n} \widehat{C}_{i}(l)$, onde $l$ é o indice do $l$-ésimo modelo de mistura. E neste exemplo como no exemplo anterior o modelo de mistura de duas distribuições Normais é o melhor modelo para os dados da tabela (6.8) com $C(1)=\prod_{i=1}^{n} \widehat{C}_{i}(1)=2.22 \times 100^{-459}$ (normal-normal), $C(2)=\prod_{i=1}^{n} \widehat{C}_{i}(2)=2.01 \times 100^{-550}$ (normal-exponencial) e $C(3)=\prod_{i=1}^{n} \widehat{C}_{i}(3)=$ $2.62 \times 100^{-489}$ (gamma-normal), o que novamente como no exemplo 1 vem sustentar a eficiência da distribuição potência exponencial.

Neste exemplo para dados com censura tipo II não conseguimos obter os estimadores de máxima verossimilhança assintóticos (EMV) para os parâmetros dos modelos (6.2.1), (6.2.2), (6.2.3) e (6.2.4), devido a sua grande complexidade. Portanto o uso de métodos Bayesianos considerando algoritmos do tipo MCMC é uma boa alternativa para esta família de modelos.

Dos intervalos de credibilidade de $95 \%$ para $\beta$ nas tabelas (6.9), (6.10), (6.11) e (6.12), observamos que há um efeito muito pequeno das dosagens do inseticida no tempo de vida do grupo mais resistente ao tratamento. 
Este resultado está de acordo com o estudo anterior de Hewlett (1974) e de Kuo e Peng (1995) considerando tempos de sobrevivência censurados por intervalos.

Observamos também à partir dos intervalos de credibilidade de $95 \%$ para $\tau$ nas tabelas (6.9), (6.11) e (6.12), que há um grande efeito dos níveis do inseticida $x_{i k}$, nas probabilidades de incidência das populações.

\subsection{Exemplo 3: Dados com Censura por Intervalo}

Considere os dados da tabela (6.13) introduzidos por Hewlett (1974). Esse conjunto de dados consiste dos tempos de sobrevivência censurados por intervalos de $n=317$ besouros machos adultos (tribolium castaneum) que foram expostos ao (pyrethrum) um conhecido inseticida. Dentre os $n=317$ insetos, $144,69,54$ e 50 foram pulverizados de pyrethrum com concentrações de $0.20,0.32,0.50$ e $0.80 \mathrm{mg} / \mathrm{cm}^{2}$, respectivamente. O logaritmo das doses (detonado por $x_{i k}$ ), $k=1,2,3,4$, são $-1.61,-1.14,-0.69$ e -0.22 respectivamente. 
Tabela 6.13: Números de besouros mortos por dia, na linha 14 temos os besouros que sobreviveram após o $13^{\underline{a}} \mathrm{dia}$

\begin{tabular}{lllll}
\hline & \multicolumn{4}{c}{$(\mathrm{x})$ Log-dose $\left(\mathrm{mg} / \mathrm{cm}^{2}\right)$} \\
\cline { 2 - 5 } $\mathbf{r}$ & -1.61 & -1.14 & -0.69 & -0.22 \\
\hline 1 & 3 & 7 & 5 & 4 \\
2 & 11 & 10 & 8 & 10 \\
3 & 10 & 11 & 11 & 8 \\
4 & 7 & 16 & 15 & 14 \\
5 & 4 & 3 & 4 & 8 \\
6 & 3 & 2 & 2 & 2 \\
7 & 2 & 1 & 1 & 1 \\
8 & 1 & 0 & 1 & 0 \\
9 & 0 & 0 & 0 & 0 \\
10 & 0 & 0 & 0 & 1 \\
11 & 0 & 0 & 0 & 0 \\
12 & 1 & 0 & 0 & 0 \\
13 & 1 & 0 & 0 & 0 \\
14 & 101 & 19 & 7 & 2 \\
\hline
\end{tabular}

$$
x_{i 1}=-1.61 \quad x_{i 2}=-1.14
$$
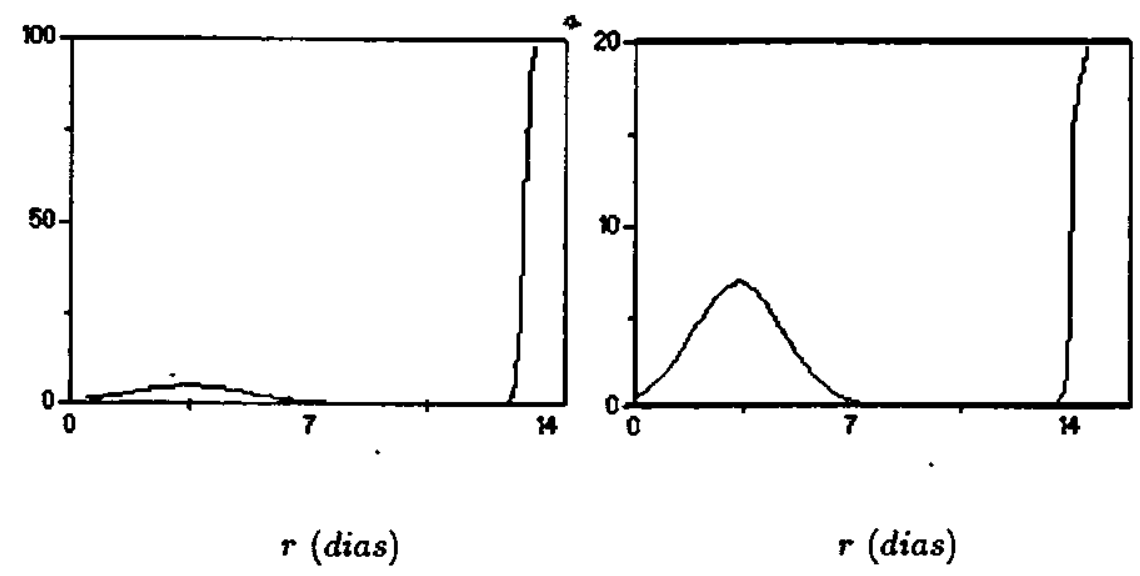

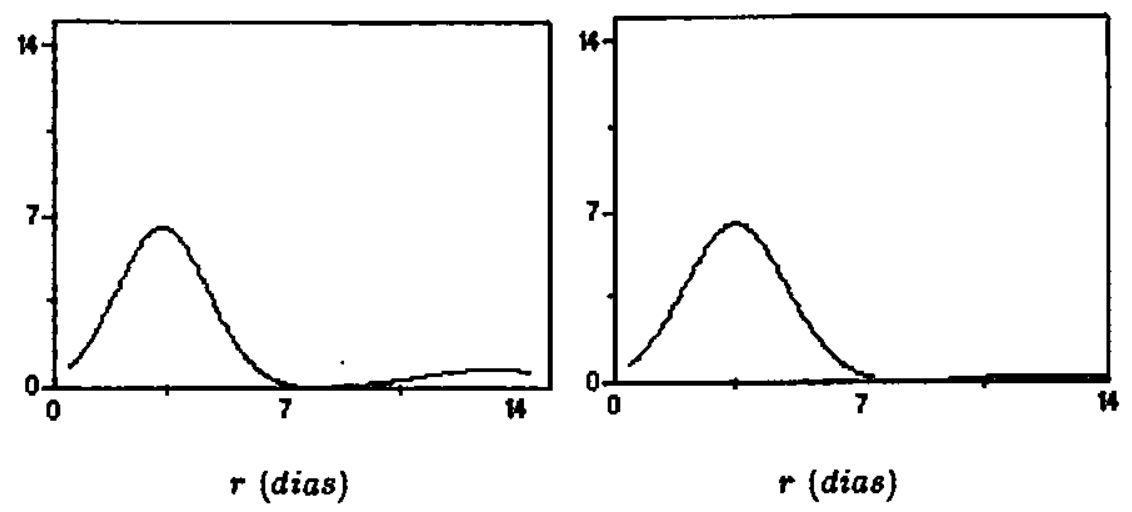

Figura 6.7: Gráfico do número de mortes considerando as 4 diferentes dosagens do inseticida $x_{i k}$

Na figura (6.7), temos os gráficos para os tempos de vida censurados por intervalos da tabela (6.13). Observamos distribuições de frequência aproximadamente simétricas para as diferentes dosagens do inseticida, especialmente para os besouros mortos antes do $13^{\underline{a}}$ dia que são mais suscetíveis ao inseticida.

\subsubsection{Mistura de Duas Distribuições Potência Exponencial}

Assumimos no modelo (1.1) a mistura de duas distribuições potência exponencial com densidades (1.4) onde $\theta_{j}=\alpha_{j}+\beta_{j} x_{i k} j=1,2$, e as probabilidades de incidência (3.4) para analisarmos os tempos de sobrevivência da tabela (6.13). Assumindo independência à priori entre os parâmetros, consideramos as densidades à priori (3.5) para $\alpha_{1}, \beta_{1}, \sigma_{1}, \delta_{1}, \alpha_{2}, \beta_{2}, \sigma_{2}, \delta_{2}, \gamma$ $\mathrm{e} \tau, \operatorname{com} \alpha_{10}=65, \sigma_{11}^{2}=14, \beta_{10}=-3.5, \sigma_{12}^{2}=7.8, \delta_{10}=0, \sigma_{13}^{2}=0.01, \delta_{20}=$ $0, \sigma_{23}^{2}=0.01, m_{11}=180, n_{11}=5.1, m_{22}=137, n_{22}=1.0, \alpha_{20}=351, \sigma_{21}^{2}=$ $3612, \beta_{20}=-127, \sigma_{22}^{2}=1573, \gamma_{0}=4, \sigma_{14}^{2}=2.5, \tau_{0}=3$ e $\sigma_{24}^{2}=2.3(\mathrm{a}$ escolha desses valores para os parâmetros das densidades à priori, foram 
baseados numa opinião experiente combinada com uma análise preliminar dos dados).

$\mathrm{Na}$ geração das variáveis latentes $w_{i}$ no algoritmo EM em cada iteração do algoritmo amostrador de Gibbs à partir de (2.4) e (2.6) com $F_{j}, j=$ 1,2 dado em (1.6), consideramos os dados censurados por intervalos em horas. Assim, para os tempos de sobrevivência da tabela (6.13) com $x_{i 1}=$ -1.61 , geramos 3 observações $w_{i}$ no intervalo $(0,24]$ à partir da densidade (2.6), para o primeiro dia. Para o segundo dia, geramos 11 observações $w_{i}$ no intervalo $(24,48]$, e para depois do $13^{\underline{a}}$ dia, geramos 101 observações $w_{i}$ censuradas à direita de $t_{i h}=14$ à partir da densidade (2.4), e assim sucessivamente para as demais covariáveis $x_{i 2}=-1.14, x_{i 3}=-0.69 \mathrm{e}$ $x_{i 4}=-0.22$.

À partir das densidades condicionais marginais (4.7) utilizando os passos (i)-(iii) do algoritmo amostrador de Gibbs (3.3) geramos 3 cadeias separadas de Gibbs cada uma com 5000 iterações, e monitoramos a convergência das amostras usando o método de Gelman e Rubin (1992). Para cada parâmetro, consideramos as iterações $10^{\underline{a}}, 20^{\underline{a}}, 30^{a}, \ldots$, com um tempo de trabalho computacional de 85 horas no software $S A S$ em um Pentium $166 \mathrm{MHZ}$. Na tabela (6.14), temos as quantidades à posteriori obtidas à partir das amostras selecionadas para cada parâmetro, temos também os estimadores dos fatores de redução de escala potencial $\widehat{R}$ (veja por exemplo, Gelman e Rubin, 1992) para todos os parâmetros. Neste caso, o número de iterações foram suficientes para atingir a convergência uma vez que $(\sqrt{\overparen{R}}<1.1)$ para cada um dos parâmetros.

Observando os intervalos de credibilidade de $95 \%$ para $\delta_{1}$ e $\delta_{2}$ na tabela (6.14), podemos considerar uma reanalise dos dados censurados por 
Tabela 6.14: Quantidades à posteriori de interesse, obtidas à partir das amostras selecionadas, considerando a mistura de duas distribuições potência exponencial para o caso de dados censurados por intervalos

\begin{tabular}{llll}
\hline Parâmetros & Média & Intervalo Credibilidade de $95 \%$ & $\hat{R}$ \\
\hline$\alpha_{1}$ & 62.14000 & $(44.35 ; 76.14)$ & 1.000241 \\
$\beta_{1}$ & -7.55000 & $(-22.12 ;-0.25)$ & 1.006190 \\
$\sigma_{1}$ & 37.53000 & $(32.26 ; 43.45)$ & 1.005455 \\
$\delta_{1}$ & 1.00500 & $(0.99 ; 1.02)$ & 1.000356 \\
$\alpha_{2}$ & 36.76000 & $(0.72 ; 152.35)$ & 1.002669 \\
$\beta_{2}$ & -368.68000 & $(-441.76 ;-310.72)$ & 1.003292 \\
$\sigma_{2}$ & 150.07000 & $(126.45 ; 170.40)$ & 1.003185 \\
$\delta_{2}$ & 0.00720 & $(-0.012 ; 0.027)$ & 1.003137 \\
$\gamma$ & 3.59000 & $(2.41 ; 4.95)$ & 1.003258 \\
$\tau$ & 3.61000 & $(2.32 ; 5.00)$ & 1.003394 \\
\hline
\end{tabular}

intervalos da tabela (6.13) assumindo uma mistura de uma distribuição exponencial dupla $\left(\delta_{1}=1 \mathrm{em}(1.4)\right)$ e uma distribuição normal $\left(\delta_{2}=\right.$ 0 em (1.4)).

\subsubsection{Mistura das Distribuições Normal e Exponencial}

Consideramos portanto para analisar os dados censurados por intervalos da tabela (6.13) a mistura de uma distribuição exponencial e uma distribuição normal com densidades (3.12) e as probabilidades de incidência (3.4) no modelo de mistura (1.1). Observe que uma população consiste de besouros suscetíveis ao inseticida enquanto que a outra consiste de besouros mais resistentes ao tratamento e portanto tende à viver mais. Portanto, a escolha de uma distribuição exponencial para o grupo mais resistente ao 
tratamento é uma boa escolha. Este modelo foi considerado também para analisar os dados da tabela (6.13) por Kuo e Peng (1995).

Assumindo independência à priori entre os parâmetros consideramos as densidades à priori (3.13) com $\gamma_{0}=4, \sigma_{11}^{2}=0.7, \tau_{0}=3.6$ e $\sigma_{12}^{2}=$ 1.1, geramos 3 cadeias de separadas de Gibbs cada uma com 5000 iterações, seguindo os passos (i)-(iii) do algoritmo de Gibbs (3.3). Para cada parâmetro consideramos as iterações $5^{\underline{a}}, 10^{\underline{a}}, 15^{\underline{a}}, \ldots$, com um tempo de trabalho computacional de 53 horas utilizando o software $S A S$ em um Pentium $166 \mathrm{MHZ}$. Na tabela (6.15), temos as quantidades à posteriori para todos os parâmetros, como por exemplo as médias, intervalos de credibilidade de $95 \%$ e estimadores dos fatores de redução de escala potencial $\widehat{R}$ (ver por exemplo, Gelman e Rubin; 1992).

Tabela 6.15: Quantidades à posteriori de interesse, obtidas à partir das amostras selecionadas, considerando a mistura das distribuições normal e exponencial para o caso de dados censurados por intervalos

\begin{tabular}{llll}
\hline Parâmetros & Média & Intervalo Credibilidade de $95 \%$ & $\widehat{R}$ \\
\hline$\alpha_{1}$ & 63.810 & $(55.94 ; 71.23)$ & 1.00008700 \\
$\beta_{1}$ & -4.340 & $(-13.24 ;-0.17)$ & 1.00000000 \\
$\sigma$ & 32.280 & $(27.53 ; 37.27)$ & 1.00112400 \\
$\alpha_{2}$ & 0.0037 & $(0.003 ; 0.0045)$ & 1.00211000 \\
$\beta_{2}$ & 0.0003 & $(0.0003 ; 0.00043)$ & 1.00000000 \\
$\gamma$ & 4.04 & $(2.38 ; 5.59)$ & 1.00015000 \\
$\tau$ & 3.61 & $(1.58 ; 5.65)$ & 1.00629900 \\
\hline
\end{tabular}

Dos intervalos de credibilidade de $95 \%$ para $\beta_{1}$ e $\beta_{2}$ na tabela (6.15), observamos que há um efeito muito pequeno das dosagens do inseticida no 
tempo de vida do grupo mais suscetível e não há efeito algum no grupo mais resistente ao tratamento. Este resultado está de acordo com o estudo anterior de Hewlett (1974) e de Kuo e Peng (1995).

Este resultado está também de acordo com os gráficos da figura (6.7). Observamos também à partir dos intervalos de credibilidade de $95 \%$ para $\tau$ na tabela (6.15), um grande efeito das dosagens do tratamento nas probabilidades de incidência das populações.

Neste exemplo como no exemplo anterior também não conseguimos obter os estimadores de máxima verossimilhança assintóticos (EMV) para os parâmetros dos modelos (6.3.1) e (6.3.2), devido a sua grande complexidade. Portanto o uso de métodos Bayesianos considerando algoritmos do tipo MCMC é uma boa alternativa para esta família de modelos considerando dados agrupados. 


\section{Capítulo 7}

\section{Conclusões e Considerações Futuras}

O uso de modelos de mistura é uma alternativa eficiente para analisar tempos de sobrevivência sem censura, com censura tipo II e censurados por intervalos, uma vez que em muitas aplicações podemos ter indivíduos que reagem de maneira diferente à um tederminado tratamento. A mistura das distribuições potência exponencial (1.4) oferece uma grande flexibilidade para analisar dados das tabelas (6.1), (6.8) e (6.13), especialmente se distribuições aproximadamente simétricas podem ser consideradas.

Uma análise baseada na teoria Bayesiana para os modelos de mistura (1.1) na presença de covariáveis usando métodos de simulação estocástica do tipo Cadeia de Markov com Monte Carlo (MCMC) tem implementação fácil, não exige um conhecimento computacional avançado e as inferências obtidas são bastante precisas. Entretanto, as inferências baseadas na teoria clássica para estes modelos podem ser em muitos casos, bastante difíceis, imprecisos ou impossíveis de se obterem.

Poderíamos ainda considerar a mistura de $J=3$ componentes no 
modelo de mistura (1.1) incorporando covariáveis de interesse, e também generalizar para mais de 3 componentes de mistura. Uma outra aplicação dos modelos de mistura (1.1), seria em análise de regressão onde por exemplo os erros fossem assumidos como misturas de normais. 


\section{Apêndice A}

\section{Função Distribuição Acumulada da Poência Exponen- cial}

Para gerar os valores de $w_{i}$ da variável artificial $W$ no algoritmo EM do algoritmo amostrador de Gibbs (3.3), precisamos conhecer as funções distribuições acumulada das densidades de latências condicionais no modelo de mistura (1.1). Em especial quando consideramos a mistura de duas distribuições potência exponecial com densidades (1.4) no modelo (1.1), utilizaremos as funções distribuições acumulada das densidades (1.4), para gerar $w_{i}$ à partir da densidade (2.4) no caso de censura tipo II e à partir da densidade (2.6) no caso de censura por intervalo. Temos que a função distribuição acumulada da potência exponencial é expressa como uma gamma incompleta, como apresentaremos a seguir.

$$
\begin{gathered}
F_{j}\left(t \mid \mathbf{x}, \beta_{\mathbf{j}}\right)=\int_{-\infty}^{t} f_{j}\left(y \mid \mathbf{x}, \beta_{\mathbf{j}}\right) d y \\
=\frac{w\left(\delta_{j}\right)}{\sigma_{j}} \int_{-\infty}^{t} \exp \left\{-c\left(\delta_{j}\right)\left|\frac{y-\theta_{j}}{\sigma_{j}}\right|^{2 /\left(1+\delta_{j}\right)}\right\} d y \\
=\frac{w\left(\delta_{j}\right)}{\sigma_{j}} \int_{-\infty}^{t} \exp \left\{-\frac{1}{2}\left[2^{1 / 2} c^{1 / 2}\left(\delta_{j}\right)\left|\frac{y-\theta_{j}}{\sigma_{j}}\right|^{1 /\left(1+\delta_{j}\right)}\right]^{2}\right\} d y
\end{gathered}
$$

Consideremo a seguinte transformação:

$$
z_{j}=\left[2 c\left(\delta_{j}\right)\right]^{1 / 2}\left|\frac{y-\theta_{j}}{\sigma_{j}}\right|^{1 /\left(1+\delta_{j}\right)}, z_{j}>0, \text { assim }
$$




$$
\begin{gathered}
d z_{j}=\left[2 c\left(\delta_{j}\right)\right]^{1 / 2} \frac{1}{\left(1+\delta_{j}\right)}\left|\frac{y-\theta_{j}}{\sigma_{j}}\right|^{\frac{1}{\left(1+\delta_{j}\right)}-1} \cdot \frac{1}{\sigma_{j}} d y \\
=\frac{\left[2 c\left(\delta_{j}\right)\right]^{1 / 2}}{\sigma_{j}\left(1+\delta_{j}\right)}\left|\frac{y-\theta_{j}}{\sigma_{j}}\right|^{\frac{-\delta_{j}}{\left(1+\delta_{j}\right)}} d y
\end{gathered}
$$

onde,

$$
\left|\frac{d y}{d z_{j}}\right|=\frac{\sigma_{j}\left(1+\delta_{j}\right)}{\left[2 c\left(\delta_{j}\right)\right]^{1 / 2}}\left|\frac{y-\theta_{j}}{\sigma_{j}}\right|^{\frac{\delta_{j}}{\left(1+\delta_{j}\right)}}
$$

ou $d y=\frac{\sigma_{j}\left(1+\delta_{j}\right)}{\left[2 c\left(\delta_{j}\right)\right]^{1 / 2}}\left\{\frac{z_{j}}{\left[2 c\left(\delta_{j}\right)\right]^{1 / 2}}\right\}^{\delta_{j}}$

Portanto,

$$
d y=\frac{\sigma_{j}\left(1+\delta_{j}\right)}{\left[2 c\left(\delta_{j}\right)\right]^{1 / 2}}\left\{\frac{z_{j}^{\delta_{j}}}{\left[2 c\left(\delta_{j}\right)\right]^{\delta_{j} / 2}}\right\}
$$

Daí,

$$
\begin{aligned}
F_{j}\left(t \mid \mathbf{x}, \beta_{\mathbf{j}}\right)= & \frac{2 w\left(\delta_{j}\right)}{\sigma_{j}} \int_{0}^{\left[2 c\left(\delta_{j}\right)\right]^{\mathrm{i} / 2}\left|\frac{y-\theta_{j}}{\sigma_{j}}\right|^{\frac{1}{\left(1+\delta_{j}\right)}}} \exp \left\{-\frac{z_{j}^{2}}{2}\right\} \frac{\sigma_{j}\left(1+\delta_{j}\right) z_{j}^{\delta_{j}}}{\left[2 c\left(\delta_{j}\right)\right]^{1 / 2}\left[2 c\left(\delta_{j}\right)\right]^{\delta_{j} / 2}} d z_{j} \\
& =\frac{2 \sigma_{j}\left(1+\delta_{j} w\left(\delta_{j}\right)\right.}{\sigma_{j}\left[2 c\left(\delta_{j}\right)\right]^{\left(\delta_{j}+1\right) / 2}} \int_{0}^{\left[2 c\left(\delta_{j}\right)\right]^{1 / 2}\left|\frac{y-\theta_{j}}{\sigma_{j}}\right|^{\frac{1}{\left(1+\delta_{j}\right)}}} z_{j}^{\delta_{j}} e^{-\frac{z_{j}^{2}}{2}} d z_{j}
\end{aligned}
$$

Consideremos agora uma segunda transformação:

$$
v_{j}=\frac{z_{j}^{2}}{2} \rightarrow d v_{j}=z_{j} d z_{j}
$$

$\operatorname{assim} d z_{j}=\frac{d v_{j}}{z_{j}}$, isto é, $d z_{j}=\frac{d v_{j}}{\left(2 v_{j}\right)^{1 / 2}}$ 
Com isso temos,

$$
\begin{gathered}
F_{j}\left(t \mid \mathbf{x}, \beta_{\mathbf{j}}\right)=\frac{2\left(1+\delta_{j} w\left(\delta_{j}\right)\right.}{\left[2 c\left(\delta_{j}\right)\right]^{\left(\delta_{j}+1\right) / 2}} \int_{0}^{\left[c\left(\delta_{j}\right)\right]\left|\frac{t-\theta_{j}}{\sigma_{j}}\right|^{\frac{2}{\left(1+\delta_{j}\right)}}\left(2 v_{j}\right)^{\delta_{j} / 2} e^{-v_{j}} \frac{d v_{j}}{\left(2 v_{j}\right)^{1 / 2}}} \\
=\frac{2\left(1+\delta_{j} w\left(\delta_{j}\right) 2^{\delta_{j} / 2} 2^{-1 / 2}\right.}{2^{\left(\delta_{j}+1\right) / 2}\left[c\left(\delta_{j}\right)\right]^{\left(\delta_{j}+1\right) / 2}} \int_{0}^{\left.\left.\left[c\left(\delta_{j}\right)\right]\right|^{\frac{t-\theta_{j}}{\sigma_{j}}}\right|^{\frac{2}{\left(1+\delta_{j}\right)}}\left(v_{j}\right)^{\delta_{j} / 2-1 / 2} e^{-v_{j}} d v_{j}}
\end{gathered}
$$

Portanto,

$$
\begin{gathered}
F_{j}\left(t \mid \mathbf{x}, \beta_{\mathbf{j}}\right)=\frac{\left(1+\delta_{j} w\left(\delta_{j}\right)\right.}{\left[c\left(\delta_{j}\right)\right]^{\left(\delta_{j}+1\right) / 2}} \int_{0}^{\left.\left[c\left(\delta_{j}\right)\right]\right|^{\left.\frac{t-\theta_{j}}{\sigma_{j}}\right|^{\frac{2}{\left(1+\delta_{j}\right)}}}\left(v_{j}\right)^{\frac{\delta_{j}+1}{2}-1} e^{-v_{j}} d v_{j}} \\
=\frac{\left(1+\delta_{j} w\left(\delta_{j}\right) \Gamma\left(\frac{\delta_{j}+1}{2}\right)\right.}{\left[c\left(\delta_{j}\right)\right]^{\left(\delta_{j}+1\right) / 2}} \int_{0}^{\left.\left.\left[c\left(\delta_{j}\right)\right]\right|^{\frac{t-\theta_{j}}{\sigma_{j}}}\right|^{\frac{2}{\left(1+\delta_{j}\right)}}} \frac{1}{\Gamma\left(\frac{\delta_{j}+1}{2}\right)}\left(v_{j}\right)^{\frac{\delta_{j}+1}{2}-1} e^{-v_{j}} d v_{j}
\end{gathered}
$$

Assim,

$$
\begin{aligned}
F_{j}\left(t \mid \mathbf{x}, \beta_{\mathbf{j}}\right)= & \left\{\frac{\left(1+\delta_{j}\right) w\left(\delta_{j}\right) \Gamma\left(\frac{\delta_{j}+1}{2}\right)}{\left[c\left(\delta_{j}\right)\right]^{\left(\delta_{j}+1\right) / 2}}\right\} \\
& \frac{I_{\frac{\left(\delta_{j}+1\right)}{2}}}{2}\left\{c\left(\delta_{j}\right)\left|\frac{t-\theta_{j}}{\sigma_{j}}\right|^{2 /\left(1+\delta_{j}\right)}\right\}
\end{aligned}
$$

onde

$$
I_{k}(s)=\frac{1}{\Gamma(k)} \int_{0}^{s} x^{k-1} e^{-x} d x,
$$

é a integral gamma incompleta. 


\section{Apêndice B}

\section{Algoritmo Metropolis-Hastings}

Quando as densidades condicionais marginais $\pi\left(\theta_{p} \mid \theta_{1}^{(1)}, \ldots, \theta_{p-1}^{(1)}, W^{(1)}, Z^{(1)}, \mathbf{x}\right)$, não apresentam uma forma padrão conhecida, uma alternativa então é o uso do algoritmo Metropolis-Hastings.

Suponhamos então que desejamos simular amostras de uma destas distribuições condicionais não padronizadas, devemos explorar uma forma conhecida de $\pi(\bullet)$ para especificar uma densidade geradora de candidatos, por exemplo, se $\pi(\Theta) \propto h(\Theta) \Psi(\Theta)$ onde $h(\Theta)$ é uma densidade conhecida que pode ser simulada e $\Psi(\Theta)$ é uniformemente limitado, daí considera$\operatorname{mos} h(\Theta)$ como sendo o núcleo de transição do algoritmo para geração de candidatos.

Neste caso a probabilidade de movimento só exige o cálculo da função $\Psi$, dado por:

$$
\pi(\bullet)=\min \left\{1, \frac{\Psi\left(\Theta^{(m+1)}\right)}{\Psi\left(\Theta^{(m)}\right)}\right\}
$$

- A respeito da escolha da locação e da escala das distribuições geradoras, devemos saber que:

- a variabilidade da densidade geradora de candidatos afeta o comportamento da cadeia em pelo menos 2 dimensões, uma é a "taxa de aceitação" (porcentagem de vezes que uma mudança é feita para um novo ponto), e a outra é a região do espaço amostral que é coberto pela cadeia; 
- se a variabilidade for muito grande, alguns dos candidatos gerados estarão distantes do valor atual e terão probabilidade pequena de aceitação (pois a ordenada do canditado é pequena em relação a ordenada perto da moda), e podendo assim convergir para um valor distante da moda. Isto acontece porque as distribuições condicionais são na maioria multimodais;

- a redução desta variabilidade corrige esse problema, mas se for muito pequena, a cadeia levará muito tempo para cobrir o suporte da densidade. 


\section{Apêndice C}

\section{Critério de Convergência}

Para verificarmos se as amostras geradas pelos algoritmos amostrador de Gibbs com Metropolis-Hastings estão realmente convergindo para uma distribuição estacionária, utilizamos um algoritmo baseado na técnica proposta por Gelman e Rubin (1992), o qual considera pelo menos $k=2$ cadeias paralelas com valores iniciais amostrados de uma distribuição bem comportada. Após as cadeias atingirem estacionariedade digamos na $n$ ésima iteração, consideramos então uma seleção $\left(\theta_{j}, \theta_{j+h}, \ldots, \theta_{j+N h}\right)$ como uma amostra aleatória da distribuição desejada. Devemos assumir $h$ razoavelmente grande de tal forma que 2 valores sucessivos de $\theta$ sejam independentes, assim teremos uma amostra iid.

A convergência da simulação iterativa é monitorada estimando o fator em que a escala da distribuição deva ser reduzida se as simulações são feitas até o limite quando o número de iterações tende para o infinito $(N \rightarrow \infty)$.

Se cada sequência tem comprimento $M+N$, descartamos as $M$ primeiras amostras e ficamos com as $\mathrm{N}$ últimas, daí calculamos:

$$
\frac{U}{N}=\frac{\sum_{i=1}^{k}\left(\bar{\theta}_{i}-\bar{\theta}_{.}\right)^{2}}{(k-1)}
$$

onde, $\bar{\theta}_{i .}$ : são as $k$ médias baseadas nas $\mathrm{N}$ últimas iterações da sequência, e, 


$$
W=\frac{\sum_{i=1}^{k} s_{i}^{2}}{k}
$$

onde,

$$
s_{i}^{2}=\frac{\sum_{i=1}^{k}\left(\bar{\theta}_{i .}-\bar{\theta}_{. .}\right)^{2}}{(N-1)}
$$

Podemos observar que $U / N$ representa a variabilidade entre as $k$ sequências e $W$ representa a média das $k$ variâncias dentro das sequências. Portanto, temos que a variância e a média da distribuição estacionária podem ser estimadas por:

$$
\begin{gathered}
\hat{\sigma}^{2}=\frac{N-1}{N} W+\frac{1}{N} U \\
\hat{\mu}=\bar{\theta} . .
\end{gathered}
$$

Portanto temos que a distribuição de $\theta$ condicionada aos dados tem aproximadamente uma distribuição $\mathrm{t}$ - Student com média $\hat{\mu}=\bar{\theta}_{\text {.. }}$ e desvio padrão dado por:

$$
s=\sqrt{\hat{v}}=\sqrt{\hat{\sigma}^{2}+\frac{U}{k N}}
$$

e graus de liberdade: 


$$
(g . l .)=\frac{2 \hat{v}^{2}}{V A R(\hat{v})}
$$

onde,

$$
\begin{aligned}
& V \widehat{A R}(\hat{v})=\left(\frac{N-1}{N}\right)^{2}\left(\frac{1}{k}\right) V A \widehat{R}\left(\hat{\sigma}^{2}\right)+\left(\frac{k+1}{k N}\right)^{2}+\left(\frac{2}{k-1}\right) U^{2}+ \\
& \left(\frac{2(k+1)(N-1)}{k N}\right) \frac{N}{k}\left[\operatorname{cov}\left(\sigma^{2}, \bar{\theta}_{i .}^{2}\right)-2 \bar{\theta}_{. .} \operatorname{cov}\left(\sigma^{2}, \bar{\theta}_{i .}\right)\right]
\end{aligned}
$$

Com as variâncias e covariâncias estimadas, podemos estimar o fator de redução de escala potencial por:

$$
\sqrt{\widehat{R}}=\sqrt{\left(\frac{\hat{v}}{W}\right)\left(\frac{(g . l .)}{(g . l .)-2}\right)}
$$

esperamos que esse valor convirja para 1 à medida que o número de iterações tende para o infinito $(N \rightarrow \infty)$, caso não ocorra, devemos considerar mais simulações para melhorar a inferência sobre a distribuição de interesse, ou seja quando $\sqrt{\widehat{R}} \approx 1$ Gelman e Rubin (1992) argumentam que as amostras selecionadas convergem em distribuição para a distribuição condicionada aos dados, e os pontos amostrais são iid. 


\section{Apêndice D}

\section{Programa Computacional}

Neste apêndice, apresentaremos um dos programas computacionais utilizados neste trabalho. Este programa foi desenvolvido usando o pacote (Procedure I.M.L.(interative matrix language) do software S.A.S versão 6.08 .

O programa apresentado é um simulador de amostras baseado nos algoritmos de simulação do tipo MCMC, Gibbs e Metropolis-Hastings. Este programa gera amostras à partir das densidades à posteriori marginais para os parâmetros do modelo de mistura (1.1) considerando a mistura de duas distribuições potência exponencial com densidade (1.4) e os tempos de sobrevivência da tabela (6.13) censurados por intervalos.

\footnotetext{
/***************

ENTRADA DE DADOS

$* * * * * * * * * * * * * * /$

data ganorag;

infile dagrupa.dat;

input $x$ til tiued;

run;

data ganorag1;

infile dagrupa1.dat;

input $x 1$ tloe;

run;
} 
data ganorag2;

infile dagrupa2.dat;

input $\times 2$ t@Q;

run;

proc iml;

/************************

LEITURA DAS VARIÁVEIS

$* * * * * * * * * * * * * * * * * * * * * * 1$

use ganorag;

read all $\operatorname{var}\{x, t i l, t i u\}$;

use ganorag1;

read all $\operatorname{var}\{x 1, t I\}$;

use ganorag2;

$\operatorname{read}$ all $\operatorname{var}\{\mathrm{x} 2, \mathrm{t}\}$

/****************************************************

VALORES DOS PARÂMETROS DAS DENSIDADES À PRIORI

$* * * * * * * * * * * * * * * * * * * * * * * * * * * * * * * * * * * * * * * * * * * * * * * * * * /$

media12 $=4.04 ; \operatorname{media} 22=3.6 ; \operatorname{sigma12}=0.7 ; \operatorname{sigma22}=1.1 ; \mathrm{m} 1=57.85 ;$ $\mathrm{s} 1=142.76 ; \mathrm{m} 2=-9.43 ; \mathrm{s} 2=76.35 ; \mathrm{m} 3=95.77 ; \mathrm{s} 3=3801.11 ; \mathrm{m} 4=-414.92$; $\mathrm{s} 4=1394.21 ;$ media $01=1 ; \operatorname{media} 02=0 ; \operatorname{sigma01}=1 \mathrm{e}-2 ; \operatorname{sigma02}=1 \mathrm{e}-2$; $a 11=180 ; b 11=5.14 ; a 22=137 ; b 22=1.01 ; \operatorname{delt} 11=1$; $\operatorname{delt} 1=1.009 ; \operatorname{delt} 22=0.0014 ; \operatorname{delt} 2=0.0014 ;$ 


\section{$/ * * * * * * * * * * * * * * * * * * * * *$ \\ CONDIÇÕES INICIAIS \\ $* * * * * * * * * * * * * * * * * * * * /$}

alfa1 $=67.9 ;$ beta1 $=-3.8 ; \operatorname{sigm1}=36.97 ;$ alfa $2=87.674$;

beta2 $=-349.46 ; \operatorname{sigm} 2=145.78 ;$ gama $=4.86 ; \operatorname{tau}=2.96 ; n=\operatorname{nrow}(x)$; maxiter $=5 ; \max i \operatorname{ter} 1=5 ; w=j(317,1,.) ; w 0=w ; w 1=w ; w 2=w ; w 3=w$; $w 4=w ; w 5=w ; w 6=w ; w 7=w ; w 8=w ; w 9=w ; w 10=w ; w g=w ; ~ w g g=w ;$ $\mathrm{v}=\mathrm{w} ; \mathrm{v} 1=\mathrm{v} ; \mathrm{pn} 0=\mathrm{w} ; \mathrm{pn} 1=\mathrm{w} ; \mathrm{pn} 2=\mathrm{w} ; \mathrm{pn} 3=\mathrm{w} ; \mathrm{pn} 4=\mathrm{w} ; \mathrm{pn} 5=\mathrm{w} ; \mathrm{ws}=\mathrm{w} ; \mathrm{wh}=\mathrm{w}$; w11=w;z1 $=w ; z 2=z 1 ; h 1=z 1 ; h 2=z 1 ; p 1=z 1 ; p 2=z 1 ;$

\section{$/ * * * * * * * * * * * * * * * * * * * * * * * * * * * * * * * * * * * * * * * * * * * * * * * * * * * *$}

ROTINA PAR GERAR $V_{i}$ NO CASO DE CENSURA POR INTERVALO $* * * * * * * * * * * * * * * * * * * * * * * * * * * * * * * * * * * * * * * * * * * * * * * * * * * * * * * * * * * /$

start vi0;

pno $[\mathrm{k}]=((2 \#(\operatorname{gamma}(3 / 2+3 / 2 \#$ delt1) $/ \operatorname{gamma}(1 / 2+d e 1 t 1 / 2)) \# \#$

$(1 /(1+\operatorname{delt} 1)) \#(\operatorname{abs}((t i l[k]-$ alfa1-beta1\#x $[k]) / \operatorname{sigm} 1) \# \#$

$(2 /(1+d e 1 t 1))))) / 2$;

$\operatorname{pn} 1[\mathrm{k}]=((2 \#(\operatorname{gamma}(3 / 2+3 / 2 \# \operatorname{delt} 1) / \operatorname{gamma}(1 / 2+\operatorname{delt1} / 2)) \# \#$

$(1 /(1+\operatorname{delt1})) \#(\operatorname{abs}((\mathrm{tiu}[\mathrm{k}]$-alfa1-beta1\#x[k])/sigm1)\#\#

$(2 /(1+\operatorname{delt} 1))))) / 2$;

pn2 $[\mathrm{k}]=((2 \#(\operatorname{gamma}(3 / 2+3 / 2 \#$ delt2 $) / \operatorname{gamma}(1 / 2+\mathrm{delt} 2 / 2)) \# \#$

$(1 /(1+\operatorname{delt2})) \#($ abs $((\mathrm{til}[\mathrm{k}]$-alfa2-beta2\#x [k])/sigm2)\#\#

$(2 /(1+d e l t 2))))) / 2$;

pn3 $[\mathrm{k}]=((2 \#(\operatorname{gamma}(3 / 2+3 / 2 \#$ delt2 $) / \operatorname{gamma}(1 / 2+$ delt2/2) $) \#$

$(1 /(1+\operatorname{delt2})) \#($ abs $((t i u[k]-a l f a 2-b e t a 2 \# x[k]) /$ sigm2)\#\#

$(2 /(1+\operatorname{de}(t 2))))) / 2$;

$v[k]=\exp ($ gama+tau\#x $[k]) /(1+\exp ($ gama $+\mathrm{tau} \# \mathbf{x}[\mathrm{k}])) \#$ 
$(\operatorname{probgam}(\mathrm{pn} 0[\mathrm{k}],(\operatorname{delt1+1}) / 2))+1 /(1+\exp ($ gama+tau\# $x[k])) \#(\operatorname{probgam}(\operatorname{pn} 2[\mathrm{k}],(\operatorname{delt} 2+1) / 2))+\operatorname{uniform}(\mathrm{k}) \#$ $((\exp ($ gama+tau\#x $[\mathrm{k}]) /(1+\exp ($ gama+tau\#x $[\mathrm{k}])) \#$ $(\operatorname{probgam}(\operatorname{pn} 1[\mathrm{k}],(\operatorname{delt1+1}) / 2))+1 /(1+\exp ($ gama+ tau\#x $[k])) \#(\operatorname{probgam}(\operatorname{pn} 3[k],(\operatorname{delt2+1)/2)))-}$ $(\exp ($ gama + tau\#x $[\mathrm{k}]) /(1+\exp ($ gama+tau\#x $[\mathrm{k}]))$

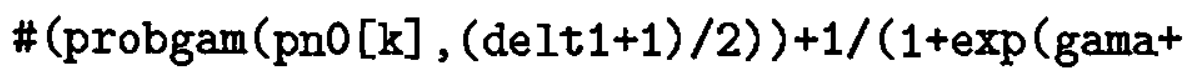

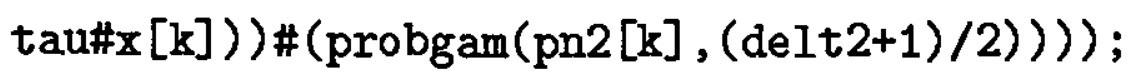

finish;

/********************************************** ROTINA PAR GERAR $V_{i}$ NO CASO DE CENSURA TIPO II *************************************************/

start vi1;

$\operatorname{pn} 1[\mathrm{k}]=((2 \#(\operatorname{gamma}(3 / 2+3 / 2 \# \operatorname{delt1}) /$

$\operatorname{gamma}(1 / 2+\operatorname{delt1} / 2)) \#(1 /(1+\operatorname{delt} 1)) \#$

(abs ( (tl $[\mathrm{k}]$-alfa1-beta1\# $1[\mathrm{k}]) /$

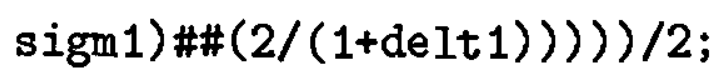

$\mathrm{pn} 2[\mathrm{k}]=((2 \#(\operatorname{gamma}(3 / 2+3 / 2 \#$ delt 2$) /$

$\operatorname{gamma}(1 / 2+\operatorname{de} 1 t 2 / 2)) \#(1 /(1+\operatorname{delt} 2)) \#$

$\operatorname{abs}((\mathrm{t} 1[\mathrm{k}]$-alfa2-beta2\#x1[k])/

$\operatorname{sigm} 2) \#(2 /(1+\operatorname{de}(t 2))))) / 2$;

$v[k]=\exp ($ gama+tau\#x1[k])/(1+exp $($ gama+tau\#x1 $[\mathrm{k}])) \#$ $(\operatorname{probgam}(\operatorname{pn} 1[\mathrm{k}],(\operatorname{delt1+1)/2)})+1 /(1+\exp ($ gama+tau\# $\mathrm{x} 1[\mathrm{k}]))$ \#(probgam $(\mathrm{pn} 2[\mathrm{k}],(\operatorname{delt} 2+1) / 2))+\operatorname{uniform}(\mathrm{k}) \#$ $(1-(\exp ($ gama+tau\#x $1[\mathrm{k}]) /(1+\exp ($ gama+tau\#x $1[\mathrm{k}])) \#$ $(\operatorname{probgam}(\operatorname{pn} 1[\mathrm{k}],(\operatorname{delt1} 1) / 2))+1 /(1+\exp ($ gama+tau\# 


$$
x 1[k])) \#(\operatorname{probgam}(\operatorname{pn} 2[k],(\operatorname{delt} 2+1) / 2)))) \text {; }
$$

finish;

\section{$/ * * * * * * * * * * * * * * * * * * * * * * * * * * * * * * * * * * * * * * * * * * * * * * * * * * * *$}

ROTINA PAR GERAR $W_{i}$ NO CASO DE CENSURA POR INTERVALO

start fun;

$w[k]=((2 \#(\operatorname{gamma}(3 / 2+3 / 2 \# \operatorname{delt} 1) / \operatorname{gamma}(1 / 2+$ $\operatorname{delt1} / 2)) \#(1 /(1+\operatorname{delt1})) \#(\operatorname{abs}((\operatorname{wgg}[\mathrm{k}]-$ alfa1-beta1\#x [k])/sigm1)\#\#(2/(1+delt1)))))/2; w0 $[\mathrm{k}]=((2 \#(\operatorname{gamma}(3 / 2+3 / 2 \# d e 1 t 2) / \operatorname{gamma}(1 / 2+$

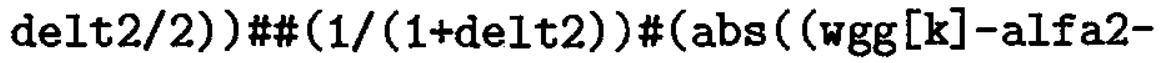
beta2\#x $[\mathrm{k}]) / \operatorname{sigm} 2) \# \#(2 /(1+\operatorname{delt} 2))))) / 2$; w7 $[\mathrm{k}]=\operatorname{probgam}(\mathrm{w}[\mathrm{k}],(\operatorname{delt} 1+1) / 2)$; w8 $[\mathrm{k}]=\operatorname{probgam}($ w0 $[\mathrm{k}],(\operatorname{delt2+1}) / 2)$; w9 $[\mathrm{k}]=\exp ($ gama+tau\#x $[\mathrm{k}]) /(1+\exp ($ gama+tau\#x $[\mathrm{k}]))$; w10 $[k]=1 /(1+\exp ($ gamattau\#x $[k]))$; w11 $[\mathrm{k}]=(($ w7 $[\mathrm{k}]) \#($ w9 $[\mathrm{k}])+($ w8 $[\mathrm{k}]) \#($ w10 $[\mathrm{k}]))$; w2 $[\mathrm{k}]=\mathrm{w} 11[\mathrm{k}]-\mathrm{v}[\mathrm{k}]$; w5 $[\mathrm{k}]=((\exp (\operatorname{gama}+\mathrm{tau} \# \mathbf{x}[\mathrm{k}]) /(1+\exp (\operatorname{gama}+\operatorname{tau} \# \mathrm{x}[\mathrm{k}]))$ \#sqrt (gamma $(3 / 2+3 / 2 \# \operatorname{delt1})) /(1+\operatorname{delt1}) /$ sqrt (gamma (1/2+delt1/2))\#\#3/sigm1\#exp (- (gamma $(3 / 2+3 / 2 \# \operatorname{delt1}) / \operatorname{gamma}(1 / 2+\operatorname{delt1} / 2)) \#(1 /(1+$ $\operatorname{delt1))\# abs~((wgg[k]-alfa1-beta1\# x[k])/sigm1)\# \# ~}$ $(2 /(1+\operatorname{delt1}))))+(\exp ($ gama+tau\#x $[k]) /(1+\exp ($ gama+tau \#x[k]))\#sqrt (gamma (3/2+3/2\#delt2))/(1+deIt2)/sqrt (gamma (1/2+delt2/2))\#3/sigm2\#exp (- (gamma (3/2+3/2 


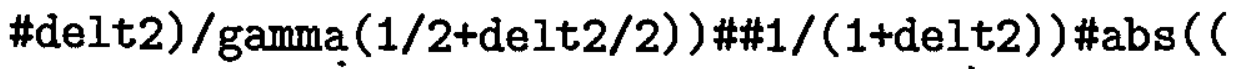
wgg [k]-alfa2-beta2\#x [k])/sigm2)\#\#(2/(1+delt2))))) +1e-2; w6 $[\mathrm{k}]=\mathrm{w} 2[\mathrm{k}] / \mathrm{w} 5[\mathrm{k}]$; w1 $[\mathrm{k}]=\mathrm{abs}$ (wgg $[\mathrm{k}]-\mathrm{w} 6[\mathrm{k}]$ );

$\mathrm{wgg}[\mathrm{k}]=\mathrm{w} 1[\mathrm{k}]$; $w g[k]=w g g[k]$;

finish;

\section{$/ * * * * * * * * * * * * * * * * * * * * * * * * * * * * * * * * * * * * * * * * * * * * * * *$}

ROTINA PAR GERAR $W_{i}$ NO CASO DE CENSURA TIPO II $* * * * * * * * * * * * * * * * * * * * * * * * * * * * * * * * * * * * * * * * * * * * * * * * * /$

start fun1;

$w[k]=((2 \#(\operatorname{gamma}(3 / 2+3 / 2 \# \operatorname{delt1}) / \operatorname{gamma}(1 / 2+\operatorname{delt1} / 2))$ $\# \#(1 /(1+\operatorname{delt1})) \#(\operatorname{abs}(($ wgg $[\mathrm{k}]-$ alfa1-beta1\#x1 [k]) $/ \operatorname{sigm} 1) \#$ \#(2/(1+delt1)))))/2; w0 $[k]=((2 \#(\operatorname{gamma}(3 / 2+3 / 2 \# \operatorname{delt} 2) / \operatorname{gamma}(1 / 2+\operatorname{delt} 2 / 2))$ \#\#(1/(1+delt2))\#(abs ((wgg[k]-alfa2-beta2\#x1[k]) /sigm2)\#\#(2/(1+delt2)))))/2; $w 7[\mathrm{k}]=\operatorname{probgam}(w[\mathrm{k}],(\operatorname{delt1+1}) / 2)$; w8 $[k]=\operatorname{probgam}(w 0[k],(\operatorname{delt} 2+1) / 2)$;

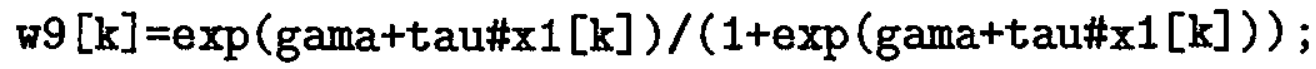
w10 $[\mathrm{k}]=1 /(1+\exp ($ gama+tau\#x1[k])); $w 11[\mathrm{k}]=\left((w 7[\mathrm{k}]) \#(w 9[\mathrm{k}])+(w 8[\mathrm{k}]) \#\left(w 10[\mathrm{k}]^{\prime}\right)\right)$; $\mathrm{w} 2[\mathrm{k}]=\mathrm{w} 11[\mathrm{k}]-\mathrm{v}[\mathrm{k}]$; $\mathrm{w} 5[\mathrm{k}]=((\exp (\operatorname{gama}+\mathrm{tau \# x1}[\mathrm{k}]) /(1+\exp (\operatorname{gama}+\mathrm{tau \# x1[k]))}$ \#sqrt (gamma $(3 / 2+3 / 2 \# \operatorname{delt1})) /(1+$ delt1)/sqrt (gamma (1/ $2+\operatorname{delt1/2)}) \# \# 3 / \operatorname{sigm1\# \operatorname {exp}}(-(\operatorname{gamma}(3 / 2+3 / 2 \# \operatorname{delt1}) /$ 
$\operatorname{gamma}(1 / 2+\operatorname{delt1} / 2)) \#(1 /(1+\operatorname{delt1})) \# a b s(($ wgg [k] alfa1-beta1\#x1 $[\mathrm{k}]) / \operatorname{sigm1}) \#(2 /(1+\operatorname{delt1}))))+(\exp ($ gama+tau\#x1[k])/(1+exp (gama+tau\#x1 [k]))\#sqrt (gamma( $3 / 2+3 / 2 \# \operatorname{delt2})) /(1+\operatorname{delt2}) / \operatorname{sqrt}(\operatorname{gamma}(1 / 2+\operatorname{delt2} / 2)) \# 3 /$ sigm2\#exp (- (gamma (3/2+3/2\#delt2)/gamma $(1 / 2+\operatorname{delt2/2)})$ \#\# (1/(1+delt2))\#abs ((wgg[k]-alfa2-beta2\#x1[k])/sigm2)\#\# $(2 /(1+\operatorname{delt2})))))+1 \mathrm{e}-1$; w6 $[\mathrm{k}]=\mathrm{w} 2[\mathrm{k}] / \mathrm{w} 5[\mathrm{k}]$; w1 $[\mathrm{k}]=\mathrm{abs}$ (wgg $[\mathrm{k}]-\mathrm{w} 6[\mathrm{k}]$ ); wgg $[\mathrm{k}]=\mathrm{w} 1[\mathrm{k}]$; $\operatorname{wg}[\mathrm{k}]=\mathrm{wgg}[\mathrm{k}]$;

finish;

$/ * * * * * * * * * * * * * * * * * * * * * * * * * * * * * * * * * * * * * * * * * * * * * * * * * *$ METODO DE NEWTON PAR GERAR $W_{i}$ (CENSURA INTERVALO) $* * * * * * * * * * * * * * * * * * * * * * * * * * * * * * * * * * * * * * * * * * * * * * * * * * * * * * * * /$

start newtono;

run fun;

do vez=1 to maxiter while $(\operatorname{abs}(w 1[k])>1 e-5)$; run fun; end;

finish;

$/ * * * * * * * * * * * * * * * * * * * * * * * * * * * * * * * * * * * * * * * * * * * * * * * * * * * * * * * * *$ ROTINA DO MÉTODO DE NEWTON PAR GERAR $W_{i}$ CENSURA TIPO II $* * * * * * * * * * * * * * * * * * * * * * * * * * * * * * * * * * * * * * * * * * * * * * * * * * * * * * * * * * * * /$ 
start newton1;

run fun1;

do vez=1 to maxiter 1

while (abs $(w 1[k])>1 e-5)$;

run fun1;

end;

finish;

$/ * * * * * * * * * * * * * * * * * * * * * * * * * * * * * * * * * * * * * * * * * * * * * * * /$

$$
\begin{aligned}
& \text { delt1out }=j(\text { iter, ncadeias, } 0) ; \\
& \text { delt2out }=j(\text { iter }, \text { ncadeias }, 0) ; \\
& \text { sigm1out }=j(\text { iter, ncadeias, } 0) \text {; } \\
& \text { alfa1out }=j(\text { iter, ncadeias, } 0) \text {; } \\
& \text { beta1out }=j(\text { iter, ncadeias, } 0) \text {; } \\
& \text { sigm2out }=\text { j(iter, ncadeias, } 0) \text {; } \\
& \text { alfa2out }=\text { j (iter, ncadeias, } 0) \text {; } \\
& \text { beta2out }=j(\text { iter }, \text { ncadeias }, 0) \text {; } \\
& \text { gamaout }=j(\text { iter }, \text { ncadeias }, 0) \text {; } \\
& \text { tauout }=j(\text { iter }, \text { ncadeias }, 0) \text {; }
\end{aligned}
$$

$/ * * * * * * * * * * * * * * * * * * * * * * * * * * * * * * * * * * * * * * * * * * * * * * * /$

ncadeias $=3$;

iter $=5000$;

burn = 1;

passo = 1

do $j=1$ to ncadeias; 


$$
\text { do } i=1 \text { to iter; }
$$

$/ * * * * * * * * * * * * * * * * * * * * *$

ALGORITMO GERADOR DE $W_{i}$

$* * * * * * * * * * * * * * * * * * * * * * * * /$

$$
\begin{aligned}
& \text { do } k=1 \text { to } 43 ; \\
& \text { run vio; } \\
& \text { end; } \\
& \text { do } k=1 \text { to } 43 ; \\
& \quad \text { vgg }[k]=t i l[k] \text {; } \\
& \quad \text { run newton0; } \\
& \text { end; }
\end{aligned}
$$

do $k=44$ to 93 ; run vio;

end;

do $k=44$ to 93 ;

$$
\text { wgg }[\mathrm{k}]=\mathrm{til}[\mathrm{k}] \text {; }
$$

run newtono;

end;

do $k=94$ to 140 ;

run vio;

end;

do $k=94$ to 140 ;

wgg $[k]=t i l[k]$; 
run newtono;

end;

do $k=141$ to 188 ;

run vio;

end ;

do $k=141$ to 188 ;

$$
\text { wgg }[\mathrm{k}]=\mathrm{til}[\mathrm{k}] \text {; }
$$

run newtono;

end;

do $k=189$ to 317 ;

run vi1;

end;

do $k=189$ to 317 ;

$$
\text { wgg }[k]=t][k] \text {; }
$$

run newton1;

end;

\section{/**********************}

ALGORITMO GERADOR DE $Z_{i}$

$* * * * * * * * * * * * * * * * * * * * * * * * * /$

$\mathrm{h} 00=(\exp ($ gama+tau\#x2) $/(1+\exp ($ gama + tau\#x2) $) \#$ $\operatorname{sqrt}(\operatorname{gamma}(3 / 2+3 / 2 \# \operatorname{delt1})) /(1+\operatorname{delt1}) / \operatorname{sqrt}(\operatorname{gamma}($

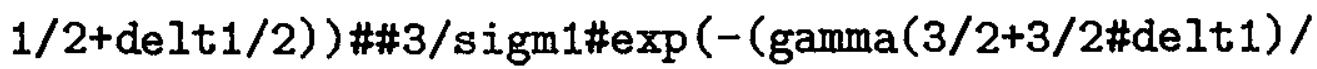
$\operatorname{gamma}(1 / 2+\operatorname{delt1} / 2)) \# \#(1 /(1+\operatorname{delt1})) \# \operatorname{abs}(($ wg-alfa1-

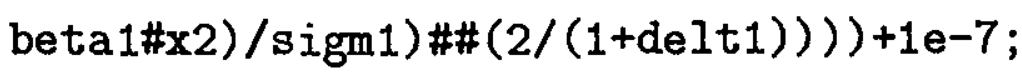




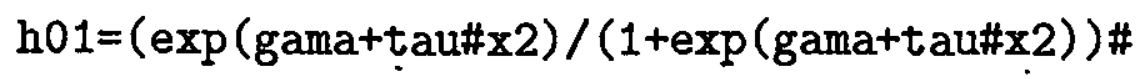
sqrt (gamma $(3 / 2+3 / 2 \# \operatorname{delt1})) /(1+\operatorname{delt1}) / \operatorname{sqrt}(\operatorname{gamma}($

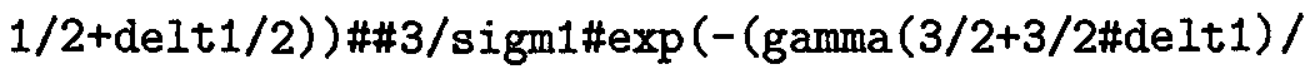
$\operatorname{gamma}(1 / 2+\operatorname{delt1} / 2))$ \#\#(1/(1+delt1))\#abs ( wg-alfa1beta1\#×2)/sigm1)\#\#(2/(1+delt1)) ) +(1/(1+exp (gama+tau\# x2)) \#sqrt (gamma (3/2+3/2\#delt2))/(1+delt2)/sqrt (gamma ( 1/2+delt2/2))\#\#3/sigm2\#exp (- (gamma (3/2+3/2\#delt2)/ $\operatorname{gamma}(1 / 2+\operatorname{delt2} / 2)) \# \#(1 /(1+\operatorname{delt2})) \# \operatorname{abs}((\mathrm{wg}-$ x2) alfa2-beta2\#/sigm2)\#\#(2/(1+delt2))))) +1e-3;

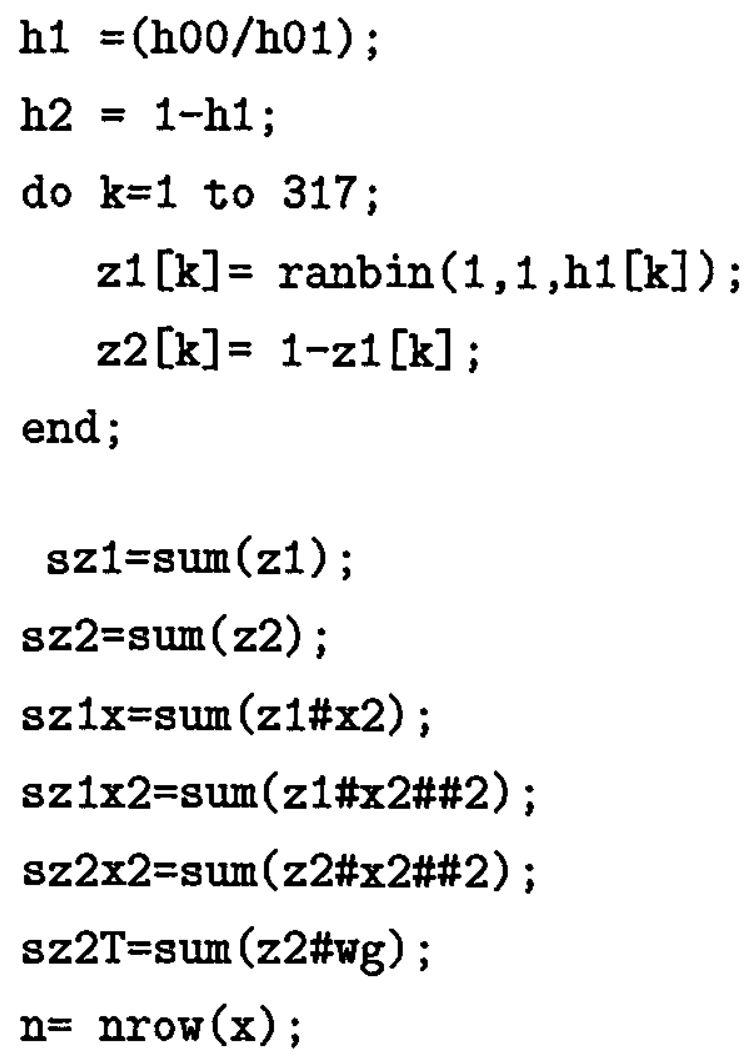




\section{$/ * * * * * * * * * * * * * * * * * * * * * * * * * * * * *$}

ALGORITMO MCMC METROPOLIS-HASTINGS

$* * * * * * * * * * * * * * * * * * * * * * * * * * * * * * * * /$

$/ * * * *$

delta $_{1}$

$* * * * * * * /$

$\operatorname{delt1c}=$ media01+ sigma01\#normal (i);

$\operatorname{prod1}=(\operatorname{sz1} \# \log (\operatorname{abs}(\operatorname{sqrt}(\operatorname{gamma}(3 / 2+3 / 2 \# \operatorname{delt1c})) /$

$(1+\operatorname{delt1c}) / \operatorname{sqrt}(\operatorname{gamma}(1 / 2+\operatorname{delt1c} / 2)) \# 3))-$

(gamma $(3 / 2+3 / 2 \# \operatorname{delt1c}) / \operatorname{gamma}(1 / 2+\operatorname{delt1c} / 2)) \#$

$(1 /(1+\operatorname{delt} 1 c)) /(\operatorname{sigm} 1 \# \#(2 /((1+\operatorname{delt1c})+1 e-5))) \#$

sum (z1\#abs ( (wg-alfa1-beta1\#x2)/sigm1)\#\#

$(2 /((1+\operatorname{delt} 1 c)))))$;

prod2 $=-($ sz1\#log $(\operatorname{abs}(\operatorname{sqrt}(\operatorname{gamma}(3 / 2+3 / 2 \# \operatorname{delt1})) /$

$(1+\operatorname{delt} 1) / \operatorname{sqrt}(\operatorname{gamma}(1 / 2+\operatorname{delt1} / 2)) \# 3))-(\operatorname{gamma}($

$3 / 2+3 / 2 \# \operatorname{delt1}) / \operatorname{gamma}(1 / 2+\operatorname{delt1} / 2)) \#(1 /(1+\operatorname{delt1})) /$

(sigm1\#\#(2/(1+delt1))) \#sum (z1\#abs ( (wg-alfa1-

beta1\#x2)/sigm1)\#\#(2/((1+delt1)))));

prod3=prod1+prod2;

phi $=\exp ($ prod3 $)+1 e-5$;

$\mathrm{aux}=\min (\mathrm{phi}, 0.99999)$;

minimo $=\operatorname{ranbin}(1,1, a u x)$;

$\operatorname{delt1}=\operatorname{delt1c\# minimo}+\operatorname{delt1\# }(1-\operatorname{minimo}) ;$ 


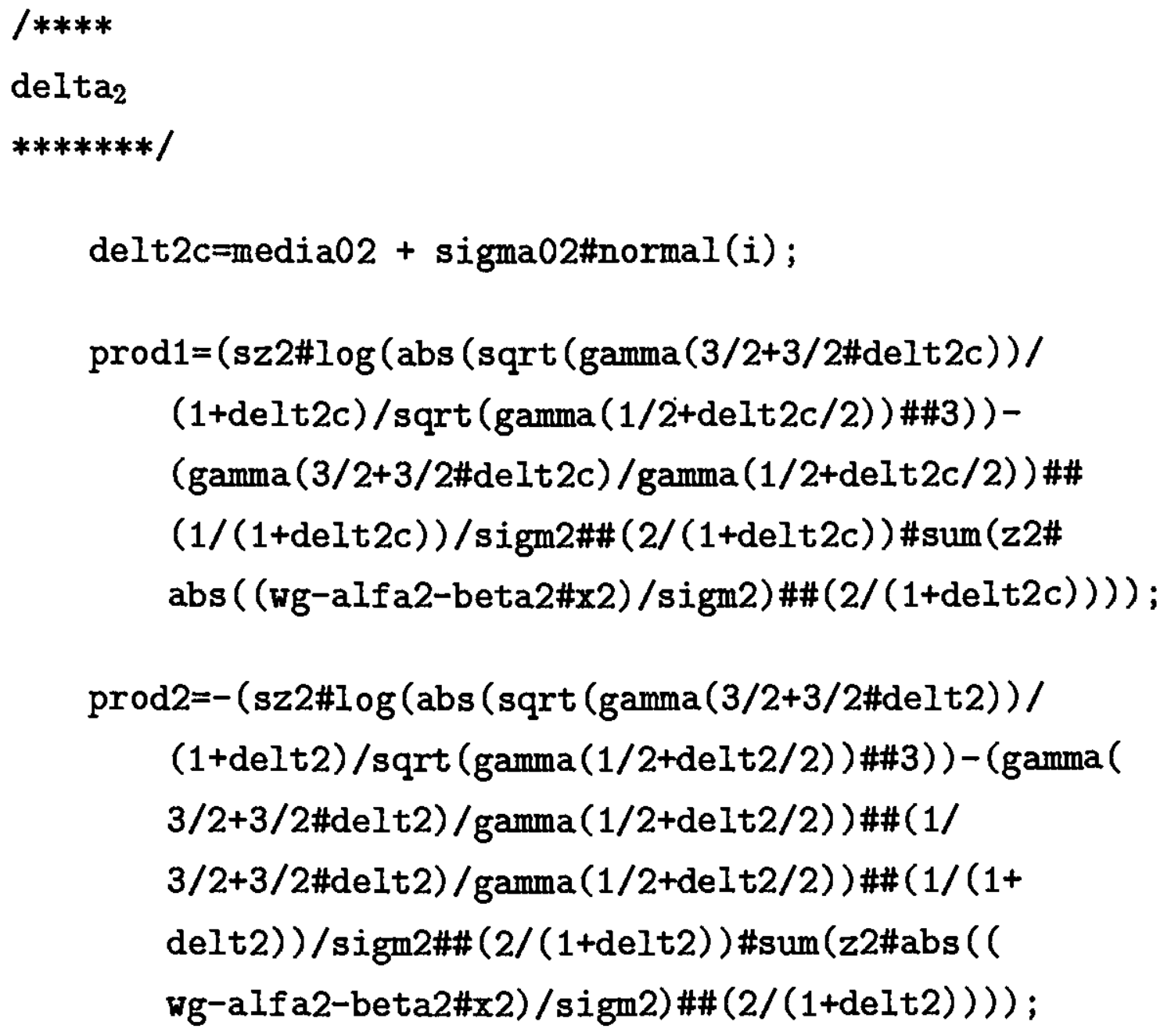

\section{/****}

alfa $_{1}$ 


$$
\operatorname{alfa1c}=m 1+\operatorname{sqrt}(s 1) \# \operatorname{normal}(i)
$$

$\operatorname{prod} 1=(-(\operatorname{gamma}(3 / 2+3 / 2 \# \operatorname{delt} 1) / \operatorname{gamma}(1 / 2+$

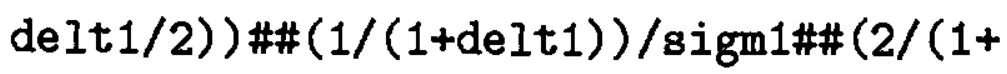

delt1)) \#sum (z1\#abs ( (wg-alfa1c-beta1\#

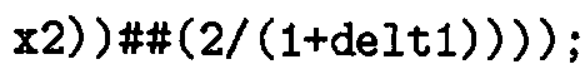

prod2 $=(($ gamma $(3 / 2+3 / 2 \# \operatorname{delt1}) / \operatorname{gamma}(1 / 2+$

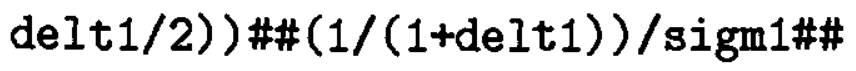

(2/(1+delt1))\#sum (z1\#abs ( (wg-alfa1beta1\#x2)) \#\#(2/(1+delt1))));

prod3=prod1+prod2;

$$
\begin{aligned}
& \text { phi=exp (prod3)+1e-5; } \\
& \text { aux }=\min (\text { phi, } 0.99999) ; \\
& \text { minimo= ranbin }(1,1, a u x) ; \\
& \text { alfa1= alfa1c\#minimo }+ \text { alfa1\#(1-minimo); }
\end{aligned}
$$

\section{/**** \\ beta $_{1}$}

beta1c $=\mathrm{m} 2+\operatorname{sqrt}(\mathrm{s} 2) \# \operatorname{normal}(\mathrm{i})$

$\operatorname{prod1}=(-(\operatorname{gamma}(3 / 2+3 / 2 \# \operatorname{delt1}) / \operatorname{gamma}(1 / 2+\operatorname{delt} 1 / 2)) \# \#(1 /(1+$

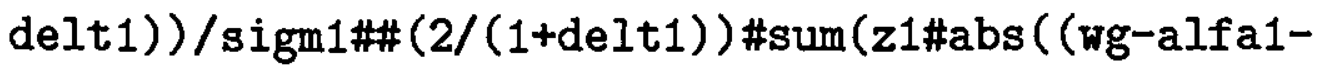
beta1c\#x2))\#\#(2/(1+delt1))));

$\operatorname{prod} 2=((\operatorname{gamma}(3 / 2+3 / 2 \# \operatorname{delt1}) / \operatorname{gamma}(1 / 2+\operatorname{delt1} / 2)) \# \#(1 /(1+$ 
$\operatorname{delt1})) / \operatorname{sigm1} \# \#(2 /(1+\operatorname{delt1})) \# \operatorname{sum}(z 1 \# a b s((w g-a l f a 1-$ beta1\#×2))\#\#(2/(1+delt1))));

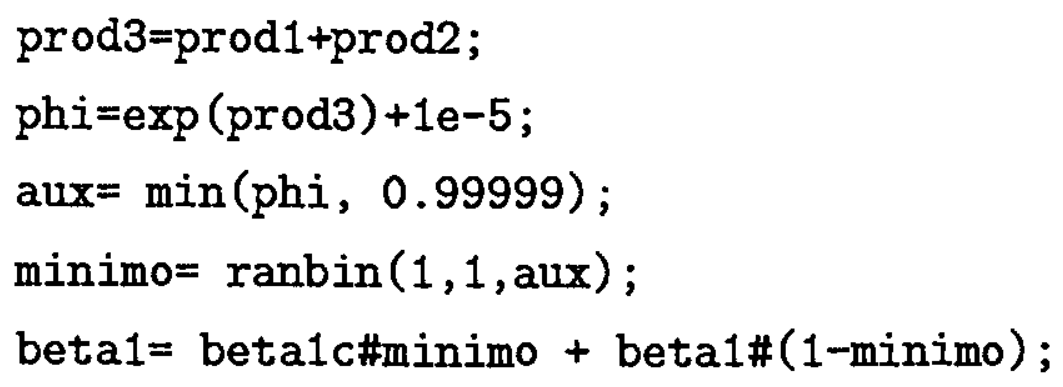

\section{/**** \\ sigma \\ $* * * * * * * /$}

$\operatorname{sigm1c}=\operatorname{rangam}(1, a 11) / b 11$

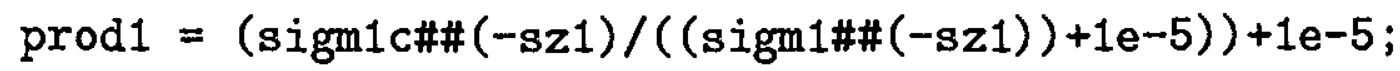

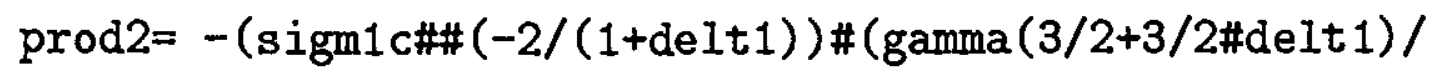
$\operatorname{gamma}(1 / 2+\operatorname{delt1} / 2)) \# \#(1 /(1+\operatorname{deIt1})) \# \operatorname{sum}(z 1 \# a b s$ ( (wgalfa1-beta1\#x2))\#\#(2/(1+delt1))));

prod3 $=(\operatorname{sigm1\# \# ~}(-2 /(1+\operatorname{delt1})) \#(\operatorname{gamma}(3 / 2+3 / 2 \# \operatorname{delt1}) /$ $\operatorname{gamma}(1 / 2+\operatorname{deIt1} / 2)) \# \#(1 /(1+\operatorname{deIt1})) \# \operatorname{sum}(z 1 \# a b s((\mathrm{wg}-$ alfa1-beta1\#xi்) \#\#(2/(1+delt1))));

prod4=prod2+prod3;

$\operatorname{prod} 5=\exp (\operatorname{prod} 4)+1 e-5 ;$

phi $=(\operatorname{prod} 1 \#$ prod 5$)+1 e-5$;

$a u x=\min (p h i, 0.99999)$; 


$$
\begin{aligned}
& \text { minimo }=\operatorname{ranbin}(1,1, \mathrm{aux}) \text {; } \\
& \text { sigm1= sigm1c\#minimo }+\operatorname{sigm1\# (1-minimo);~}
\end{aligned}
$$

\section{$/ * * * *$ \\ alfa2 \\ $* * * * * * * /$}

alfa2c $=m 3+\operatorname{sqrt}(s 3) \# \operatorname{normal}(i)$;

$\operatorname{prod} 1=(-(\operatorname{gamma}(3 / 2+3 / 2 \# \operatorname{delt2}) / \operatorname{gamma}(1 / 2+\operatorname{delt} 2 / 2)) \# \#$

$(1 /(1+\operatorname{delt2})) / \operatorname{sigm} 2 \#(2 /(1+\operatorname{delt2})) \# s u m(z 2 \# a b s((\mathrm{wg}-$ alfa2c-beta2\#x2))\#\#(2/(1+delt2))));

$\operatorname{prod} 2=((\operatorname{gamma}(3 / 2+3 / 2 \# \operatorname{delt} 2) / \operatorname{gamma}(1 / 2+\operatorname{delt} 2 / 2)) \# \#(1 /$ $(1+\mathrm{delt2})) / \operatorname{sigm} 2 \#(2 /(1+\operatorname{delt2}))$ \#sum (z2\#abs ( wg-alfa2beta2\#x2))\#\#(2/(1+delt2))));

prod3=prod1+prod2;

phi $=\exp ($ prod 3$)+1 e-5$;

$a u x=\min (p h i, 0.99999)$;

$\operatorname{minimo}=\operatorname{ranbin}(1,1, a u x)$;

alfa2= alfa2c\#minimo + alfa2\# (1-minimo);

\section{/****}

beta 2

$* * * * * * * 1$

beta2c $=m 4+\operatorname{sqrt}(s 4) \# \operatorname{normal}(i)$

$\operatorname{prod} 1=(-(\operatorname{gamma}(3 / 2+3 / 2 \# \operatorname{del} t 2) / \operatorname{gamma}(1 / 2+\operatorname{del} t 2 / 2)) \#(1 /$ 
$(1+$ delt2)) /sigm2\#\#(2/(1+delt2)) \#sum (z2\#abs ( wg-alfa2beta2c\#×2))\#\#(2/(1+delt2))));

$\operatorname{prod} 2=((\operatorname{gamma}(3 / 2+3 / 2 \# \operatorname{delt} 2) / \operatorname{gamma}(1 / 2+\operatorname{delt} 2 / 2)) \# \#(1 /(1+$ $\operatorname{delt2})$ )/sigm2\#\#(2/(1+delt2)) \#sum (z2\#abs ((wg-alfa2beta2\#x2))\#\#(2/(1+delt2))));

prod3=prod1+prod2;

$\operatorname{ph} i=\exp (\operatorname{prod} 3)+1 \mathrm{e}-5$;

aux $=\min (\mathrm{phi}, 0.99999)$;

$\operatorname{minimo}=\operatorname{ranbin}(1,1, \mathrm{aux})$;

beta2= beta2c\#minimo + beta2\# (1-minimo);

\section{/**** \\ sigma 2 \\ $* * * * * * * /$}

$\operatorname{sigm} 2 c=\operatorname{rangam}(1, a 22) / b 22$;

$\operatorname{prod} 1=(\operatorname{sigm} 2 c \# \#(-s z 2) /((\operatorname{sigm} 2 \# \#(-s z 2))+1 e-5))+1 e-5 ;$

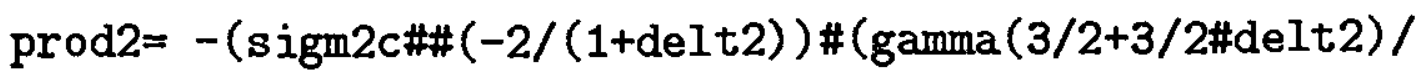

gamma $(1 / 2+\operatorname{delt2} / 2)) \#(1 /(1+\operatorname{delt} 2)) \# \operatorname{sum}(z 2 \#$ $\operatorname{abs}(($ wg-alfa2-beta2\#x2))\#\#(2/(1+delt2))));

$\operatorname{prod} 3=(\operatorname{sigm} 2 \# \#(-2 /(1+\operatorname{delt} 2)) \#(\operatorname{gamma}(3 / 2+3 / 2 \# \operatorname{delt} 2) / \operatorname{gamma}($

$1 / 2+\operatorname{delt2} / 2)) \#(1 /(1+\operatorname{delt} 2)) \#$ sum $(z 2 \#$

abs $(($ wg-alfa2-beta2\#x2)) \#\#(2/(1+delt2)))) ;

prod4=prod2+prod3; 


$$
\begin{aligned}
& \text { prod5=exp }(\text { prod4 })+1 e-5 ; \\
& \text { phi= (prod1\#prod5) }+1 e-5 ; \\
& \text { aux }=\min (\text { phi }, 0.99999) ; \\
& \text { minimo= ranbin }(1,1, \text { aux }) ; \\
& \text { sigm2= sigm2c\#minimo + sigm2\#(1-minimo); }
\end{aligned}
$$

\section{/****}

gamma

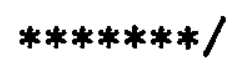

$\operatorname{gamac}=\operatorname{media12}+\operatorname{sqrt}(\operatorname{sigma12}) \# \operatorname{normal}(j) ;$

$\operatorname{prod} 1=\operatorname{sz1\# gamac}+(\operatorname{sum}(\log (1+\exp (\operatorname{gama}+\operatorname{tau} \# \mathbf{x} 2)))) ;$

$\operatorname{prod} 2=\operatorname{sz} 1 \#$ gama $+(\operatorname{sum}(\log (1+\exp (\operatorname{gamac}+\operatorname{tau} \# 2)))) ;$

prod3 $=$ prod $1-$ prod 2 ;

phi $=\exp ($ prod 3$)+1 e-5$;

aux $=\min (\mathrm{phi}, 0.99999)$;

$\operatorname{minimo2}=\operatorname{ranbin}(1,1, \mathrm{aux})$;

gama = gamac\#minimo2 + gama\#(1-minimo2);

\section{/****}

tau

\section{$* * * * * * * 1$}

$$
\begin{aligned}
& \text { tauc }=\operatorname{media} 22+\operatorname{sqrt}(\operatorname{sigma22}) \# \operatorname{mormal}(j) ; \\
& \operatorname{prod} 1=\operatorname{sz} 1 x \# \operatorname{tauc}+(\operatorname{sum}(\log (1+\exp (\operatorname{gama}+\operatorname{tau} \# x 2)))) ;
\end{aligned}
$$




$$
\begin{aligned}
& \text { prod2 = sz1x\#taut }(\operatorname{sum}(\log (1+\exp (\text { gama+tauc\#x2) }))) ; \\
& \text { prod3= prod1-prod2; } \\
& \text { phi = exp(prod3 })+1 e-5 ; \\
& \text { aux = min (phi, } 0.99999) ; \\
& \text { minimo2= ranbin }(1,1, \text { aux }) ; \\
& \text { tau= tauc\#minimo2 + tau\#(1-minimo2); }
\end{aligned}
$$

$\mid * * * * * * * * * * * * * * * * * * * * * * * * * * * * * * * * * * * * * * * * * * * * * * * /$

$$
\begin{aligned}
& \operatorname{delt1out}[i, j]=\operatorname{delt} 1 ; \\
& \operatorname{delt2out}[i, j]=\operatorname{delt} 2 ; \\
& \operatorname{sigm1out}[i, j]=\operatorname{sigm} 1 ; \\
& \operatorname{alfa1out}[i, j]=\operatorname{alfa} 1 ; \\
& \operatorname{beta1out}[i, j]=\operatorname{beta1} ; \\
& \operatorname{sigm2out}[i, j]=\operatorname{sigm} 2 ; \\
& \operatorname{alfa2out}[i, j]=\operatorname{alfa} 2 ; \\
& \operatorname{beta2out}[i, j]=\operatorname{beta} 2 ; \\
& \operatorname{gamaout}[i, j]=\operatorname{gama} ; \\
& \operatorname{tauout}[i, j]=\operatorname{tau} ;
\end{aligned}
$$

end;

end;

$/ * * * * * * * * * * * * * * * * * * * * * * * * * * * * * * * * * * * * * * * * * * * * * * * /$

$\operatorname{delt1out}=\operatorname{delt1out}[\operatorname{do}($ burn, iter,passo), ]; $\operatorname{delt2out}=\operatorname{del} t 2$ out $[$ do $($ burn, iter,passo $)$,$] ;$ 
sigm1out = sigm1out [do (burn, iter, passo ), ];

alfa1out $=$ alfa1out $[$ do (burn, iter,passo $)$,$] ;$

beta1out $=$ beta1out $[$ do (burn, iter,passo $)$,$] ;$

sigm2out $=$ sigm2out $[$ do $($ burn, iter, passo $)$,$] ;$

alfa2out $=$ alfa2out $[$ do (burn, iter,passo $)$,$] ;$

beta2out $=$ beta2out $[$ do $($ burn, iter, passo $)$,$] ;$

gamaout $=$ gamaout $[$ do $($ burn, iter, passo $)$,$] ;$

tauout $=$ tauout $[$ do $($ burn, iter, passo $)$,$] ;$

\section{/****************}

\section{ARQUIVO EXTERNO *.DAT}

$* * * * * * * * * * * * * * * * * * * /$

create saidal from delt1out;

append from delt1out;

create saida2 from delt2out;

append from delt2out;

create saida3 from sigm1out;

append from sigm1out;

create saida4 from alfalout;

append from alfa1out;

create saida5 from betalout;

append from beta1out;

create saida6 from sigm2out;

append from sigm2out; 
create saida7 from alfa2out;

append from alfa2out;

create saida8 from beta2out;

append from beta2out;

create saida9 from gamaout;

append from gamaout;

create saida10 from tauout;

append from tauout;

quit;

$\mid * * * * * * * * * * * * * * * * * * * * * * * * * * * * * * * * * * * * * * * * * * * * * * /$

data_null_;

set saida1;

file delt1. dat;

put col1 col2 col3;

run;

data_nul1_;

set saida2;

file delt2.dat;

put col1 col2 col3;

run;

data_nul1_;

set saida3;

file sigm1.dat; 
put col1 col2 col3;

run;

data_null_;

set saida4;

file alfa1.dat;

put col1 col2 col3;

run;

data_null_;

set saida5;

file beta1.dat;

put col1 col2 col3;

run;

data_null_;

set saida6;

file sigm2.dat;

put col1 col2 col3;

run;

data_null_;

set saida7;

file alfa2.dat;

put col1 col2 col3;

run;

data_null_;

set saida8; 
file beta2.dat; .

put col1 col2 col3;

run;

data_null_;

set saida9;

file gama.dat;

put col1 col2 col3;

run;

data_null.;

set saida10;

file tau.dat;

put col1 col2 col3;

run; 


\section{Bibliografia}

Achcar, J.A., (1989). Reparametrizations and Laplace approximations for posterior moments in binomial models. Rev.Mat.Estat., São Paulo, 7:73-86.

Boos, D.D.; Brownie, C. (1991). Mixture models for continuous data in dose- response studies when some animals are unaffected by treatment. Biometrics,47, 1489-1504.

Box, G.E.P.; Tiao, G.C.(1973). Bayesian inference in statistical analysis, Addison-Wesley Publishing Company.

Box, G.E.P. (1980). Sampling and Byes' inference in scientific modeling and robustness (with discussion). Journal of the Royal Statistical Society B, 143, 383-430.

Chib, S.; Greenberg, E. (1993). Markov chain Monte Carlo simulation methods in econometrics, manuscript.

Chib, S.; Greenberg, E. (1994). Bayes inference for regression models with $\operatorname{ARMA}(p, q)$ errors. Journal of Econometrics, 64, 183-206.

Cox, D.R. (1970). The Analysis of Binary Data. London: Methuen.

Cox, D.R.; Oakes, D. (1984). Analysis of survival data. London: Chapman and Hall.

Dempster, A.; Laird, N.; Rubin, D. (1977). Maximum likelihood from incomplete data via the EM algorithm. Journal of the Royal Statistical Society, series B, 39, 1-38. 
Dey, D.; Kuo, L.; and Sahu, S. (1995). A Bayesian predictive approach to determining the number of components in a mixture distribution. Statistics and Computing 5, 297-305.

Dey, D.; Peng, F. (1995). A mixture-model approach to the analysis of survival data. Technical Report Number 95-31,dep. of Statistics, University of Connecticut, Storrs.

Diebolt, J.; Robert, C. (1994). Estimation of finite mixture distributions through Bayesian sampling. Journal of the Royal Statistical Society, Series B, 56, 363-375.

Dickinson, J.P.(1974). On the resolution of a mixture of observations from two gamma distributions by the method of maximum likelihood. Metrika, 21, 133-141.

Everit, B.; Hand, D. (1981). Finite Mixture Distributions. London: Chapman and Hall.

Farewell, V.T. (1982). The use of mixture models for the analysis of survival data with long-term survivors. Biometrics, 38, 1041-1046.

Gelfand, A.E.; Smith, A.F.M. (1990) . Sampling based approaches to calculating marginal densities. Journal of the American Statistical Association, 85, 398-409.

Gelfand, A.E.; Smith, A.F.M.; Lee, T.M. (1992). Bayesian analysis of constrained parameter and truncated data problems using Gibbs sampling. Journal of the American Statistical Association,87, 523532.

Gelfand, A.E.; Dey, D.K., e Chang, H. (1992). Model determination using predctive distribution with implementation via sampling-basead 
methods (with discussion). Bayesian Statistcs 4, eds. J.M. Bernardo, J.O. Berger, A.P. Dawid, e A.F.M. Smith, Oxford: Oxford University Press, 147-169..

Geman, S.; Geman, D. (1984). Stochastic relaxation, Gibbs distributions and the Bayesian restoration of images. IEEE Transactions on Pattern Analysis and Machine Intelligence, 6, 721-741.

Gelman, A. (1992). Iterative and non-iterative simulation algorithms in Computing Science and Statistics (Iterface Proceedings), 24, 433-438.

Gelman, A.; Rubin, D.B. (1992) . Inference from iterative simulation using multiple sequences (with discussion). Statistical Science, 7,457511.

Geweke, J. (1989). Bayesian inference in econometric models using Monte Carlo and their applications, Biometrika, 57, 97-109.

Hastings, W.K. (1970). Monte Carlo sampling methods using Markov chains, and their applications. Biometrika, 57,97-109.

Hewlett, P.S. (1974). Time from dosage do death in beetles Tribolium castaneum, treated with pyrethrins or DDT, and its bearing on dosemortality relations. Journal of Stored Product Research, 10, 27-41.

Kalbfleisch, J.G. (1985). Probability and Statistical Inference (2nd edition ), New York: Springer-Verlag.

Kuk, A.Y.C.; Chen, C.H. (1992). A mixture model combining logistic regression with proportional hazards regression. Biometrika, 79, 531-541. 
Kuo, L.; Smith, A.F.M. (1992). Bayesian computations in survival models via the Gibbs samplers (with Discussion). In Survival Analysis: State of the Art J.P.klein and P.K.Goel (eds), 11-14. Dordrecht: Kluwer Academic.

Kuo, L.; Peng, F. (1995) . A mixture-model approach to the analysis of survival data. Technical Report Number 95-31, dep. of Statistics, University of Connecticut, Storrs.

Larson, M.G.; Dinse, G. (1986). A mixture model for the regression analysis of competing risks data. Applied Statistics, 34, 201-211.

Lehmann, E.L.(1983). Theory of Point Estimation. New York: Wiley.

McLachlan, G.J.; Basford, K.E. (1988). Mixture Models: Inference and Applications to Clustering. Marcel Dekker, New York.

McLachlan, G.J.; Jones P.N. (1988). Fitting mixtures models to grouped an truncated data via the EM algorithm. Biometrics, 44, 571-578.

McCullagh, P.; Nelder, J.A. (1989). Generalized Linear Models. London: Chapman and Hall.

Mendenhall, W.; R.J. Hader (1958). Estimation of parameters of mixed exponential distributed failure times from censored life test data. Biometrika, 45, 504-520.

Metropolis, N.; Rosenbluth, A.W.; Teller, A.H.; Teller, E. (1953). Equations of state calculations by fast computing machines. Journal of Chemical Physics, 21, 1087-1092.

Müller, P. (1993). A generic approach to posterior integration an Gibbs sampling, manuscript. 
Naylor, J.C. e Smith, A.F.M. (1982). Application of a method for the efficient computation of posterior distribution. Applied Statistics, $31,214-225$.

Pack, S.E.; Morgan, B.J.T. (1990). A mixture model for interval-censored time-to-response quantal assay data. Biometrics, 46, 749-757.

Phillips, D.B.; Smith, A.F.M. (1994). Bayesian faces via hierarchical template modeling. Journal of the American Statistical Association, 89, 1151-1163.

Quandt, R.E.;J.B. Ramsey (1978). Estimating mixtures of normal distributions and switching regressions (with discussion). J.Am.Stat.Assoc., $73,730-752$.

Robert, C.P. (1996) Mixture of distributions: inference and estimation, in Markov Chain Monte Carlo in practice (eds Gilks, W.R.et al), Chapman and Hall, 441-464.

S.A.S. Institute Inc. S.A.S I.M.L. User's Guide For Personal Computers, Version 6 Edition (1985). Cary, N.C.: S.A.S.Inst.Inc.

Smith, A.F.M.; Roberts, G.O. (1993) . Bayesian computation via the Gibbs sampler and related Markov Chain Monte Carlo methods, Journal of the Royal Statistical Society, B, 55, 3-24.

Tallis, G.M.;R.Light (1968). The use of fraction moments for estimating the parameters of a mixed exponential distribution. Technometrics, 10, 161-175.

Tanner, M.A.; Wong, W.H. (1987). The calculation of posterior distributions via data augmentation (with discussion), Journal of the American Statistical Association, 82, 528-550. 
Tanner, M.A.(1993). Tools for Statistical Inference (2nd edition), New York: Springer-Verlag.

Taylor, J. (1994). Semi-parametric estimation in failure time mixture models.Preprint.

Tierney, L. (1994). Markov chains for exploring posterior distributions (with discussion), Annals of Statistics forthcoming.

Tierney, L; Kadane, J.B. (1986). Accurate approximations for posterior moments and marginal densities. J.A,.Stat.Assoc.. 81, 82-86.

Titterington, D.M.; Smith, A.F.M.; and Makov, U.E. (1985) . Statistical Analysis of Finite Mixture Distributions. New York: John Wiley.

Yang, T.Y. (1994). Computational approaches to Bayesian inference for software realiability. Ph.D. Thesis, Department of Statistics, University of Connecticut, Storrs. 